\title{
Image Matching from Handcrafted to Deep Features: A Survey
}

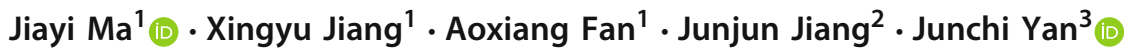

Received: 9 January 2020 / Accepted: 15 July 2020 / Published online: 4 August 2020

(c) The Author(s) 2020

\begin{abstract}
As a fundamental and critical task in various visual applications, image matching can identify then correspond the same or similar structure/content from two or more images. Over the past decades, growing amount and diversity of methods have been proposed for image matching, particularly with the development of deep learning techniques over the recent years. However, it may leave several open questions about which method would be a suitable choice for specific applications with respect to different scenarios and task requirements and how to design better image matching methods with superior performance in accuracy, robustness and efficiency. This encourages us to conduct a comprehensive and systematic review and analysis for those classical and latest techniques. Following the feature-based image matching pipeline, we first introduce feature detection, description, and matching techniques from handcrafted methods to trainable ones and provide an analysis of the development of these methods in theory and practice. Secondly, we briefly introduce several typical image matching-based applications for a comprehensive understanding of the significance of image matching. In addition, we also provide a comprehensive and objective comparison of these classical and latest techniques through extensive experiments on representative datasets. Finally, we conclude with the current status of image matching technologies and deliver insightful discussions and prospects for future works. This survey can serve as a reference for (but not limited to) researchers and engineers in image matching and related fields.
\end{abstract}

Keywords Image matching $\cdot$ Graph matching $\cdot$ Feature matching $\cdot$ Registration $\cdot$ Handcrafted features $\cdot$ Deep learning

\section{Introduction}

Communicated by V. Lepetit.

Junchi Yan

yanjunchi@sjtu.edu.cn

Jiayi Ma

jyma2010@gmail.com

Xingyu Jiang

jiangx.y@whu.edu.cn

Aoxiang Fan

fanaoxiang@whu.edu.cn

Junjun Jiang

junjun0595@163.com

1 Electronic Information School, Wuhan University, Wuhan 430072, China

2 School of Computer Science and Technology, Harbin Institute of Technology, Harbin 150001, China

3 Department of Computer Science and Engineering, and MoE Key Lab of Artificial Intelligence, AI Institute, Shanghai Jiao Tong University, Shanghai 200240, China
Vision-based artificial systems, as widely used to guide machines to perceive and understand the surroundings for better decision making, have been playing a significant role in the age of global automation and artificial intelligence. However, how to process the perceived information under specific requirements and understand the differences and/or relationships among multiple visual targets are crucial topics in various fields, including computer vision, pattern recognition, image analysis, security, and remote sensing. As a critical and fundamental problem in these complicated tasks, image matching, also known as image registration or correspondence, aims to identify then correspond the same or similar structure/content from two or more images. This technique is used for high-dimensional structure recovery as well as information identification and integration, such as 3-D reconstruction, visual simultaneous localization and mapping (VSLAM), image mosaic, image fusion, image retrieval, target recognition and tracking, as well as change detection, etc. 
Image matching has rich meaning in pairing two objects, thus deriving many specific tasks, such as sparse feature matching, dense matching (like image registration and stereo matching), patch matching (retrieval), 2-D and 3-D point set registration, and graph matching. Image matching in general consists of two parts, namely, the nature of the matched features and the matching strategy, which indicate what are used to match and how to match them, respectively. The ultimate goals are to geometrically warp the sensed image into the common spatial coordinate system of the reference image and align their common area pixel-to-pixel (i.e., image registration). To this end, a direct strategy, also known as area-based method, registers two images by using the similarity measurement of the original image pixel intensity or information after pixel-domain transformation in the sliding windows of predefined size or even the entire images, without attempting to detect any salient image structure.

Another classic and widely adopted pipeline called featurebased method, i.e., feature detection and description, feature matching, transform model estimation, image resampling and transformation, has been introduced in the prestigious survey paper (Zitova and Flusser 2003) and applied in various fields. The feature-based image matching is popular due to its flexibility and robustness and the capability of wide range applications. In particular, feature detection can extract the distinctive structure from an image, and feature description may be regarded as an image representation method that is widely used in image coding and similarity measurements such as image classification and retrieval. In addition, due to the strong ability in deep feature acquisition and non-linear expression, applying deep learning techniques for image information representation and/or similarity measurement, as well as parameter regression of image pair transformation, are hot topics in nowadays image matching community, which have been proven to achieve better matching performance and present greater potential compared with traditional methods.

In real-world settings, images for matching are usually taken from the same or similar scene/object while captured at different times, from different viewpoints or imaging modalities. In particular, a robust and efficient matching strategy is desirable to establish correct correspondences, thus stimulating various methods for achieving better efficiency, robustness and accuracy. Although numerous techniques have been devised over the decades, developing a unified framework remains a challenging task in terms of the following aspects:

- Area-based methods that directly match images often depend on an appropriate patch similarity measurement for creating pixel level matches between images. They can be computational expensive and are sensitive to image distortion, appearance changes by noise, vary- ing illumination, and different imaging sensors, which can have negative impact on similarity measurement and match searching. As a result, usually these methods can only work well under small rotation, scaling, and local deformation.

- Feature-based matching methods are often more efficient and can better handle geometrical deformation. But they are based on salient feature detection and description, feature matching, and geometrical model estimation which can also be challenging. On the one hand, in featurebased image matching, it is difficult to define and extract a high percentage and a large number of features belonging to the same positions in 3-D space in the real world to ensure the matchability. On the other hand, matching $N$ feature points to $N$ feature points detected in another image would create a total of $N$ ! possible matchings, and thousands of features are usually extracted from highresolution images and dominated outliers and noise are typically included in the points sets, which lead to significant difficulties for existing matching methods. Although various local descriptors have been proposed and coupled with detected features to ease the matching process, the use of local appearance information will unavoidably result in ambiguity and numerous false matches, especially for images with low quality, repeated contents, and those undergoing serious nonrigid deformations and extreme viewpoint changes.

- A predefined transformation model is often required to indicate the geometrical relation between two images or point sets. But it may vary on different data and is unknown beforehand thus hard to model. A simple parametric model is often insufficient for image pairs that involve non-rigid transformations caused by ground surface fluctuation and image viewpoint variations, multi-targets with different motion properties, and also local distortions.

- The emergence of deep learning has provided a new way and has shown great potential to address image matching problems. However, it still faces several challenges. The option of learning from images for direct registration or transformation model estimation is limited when applied to wide baseline image stereo or registration under complex and serious deformation. The application of convolutional neural networks (CNNs) onto sparse point data for matching, registration, and transformation model estimation is also difficult, because the points to be matched-known as unstructured or non-Euclidean data due to their disordered and dispersed nature-make it difficult to operate and extract the spatial relationships between two or more points (e.g., neighboring elements, relative positions, and length and angle information among multi-points) using a deep convolutional technique. 


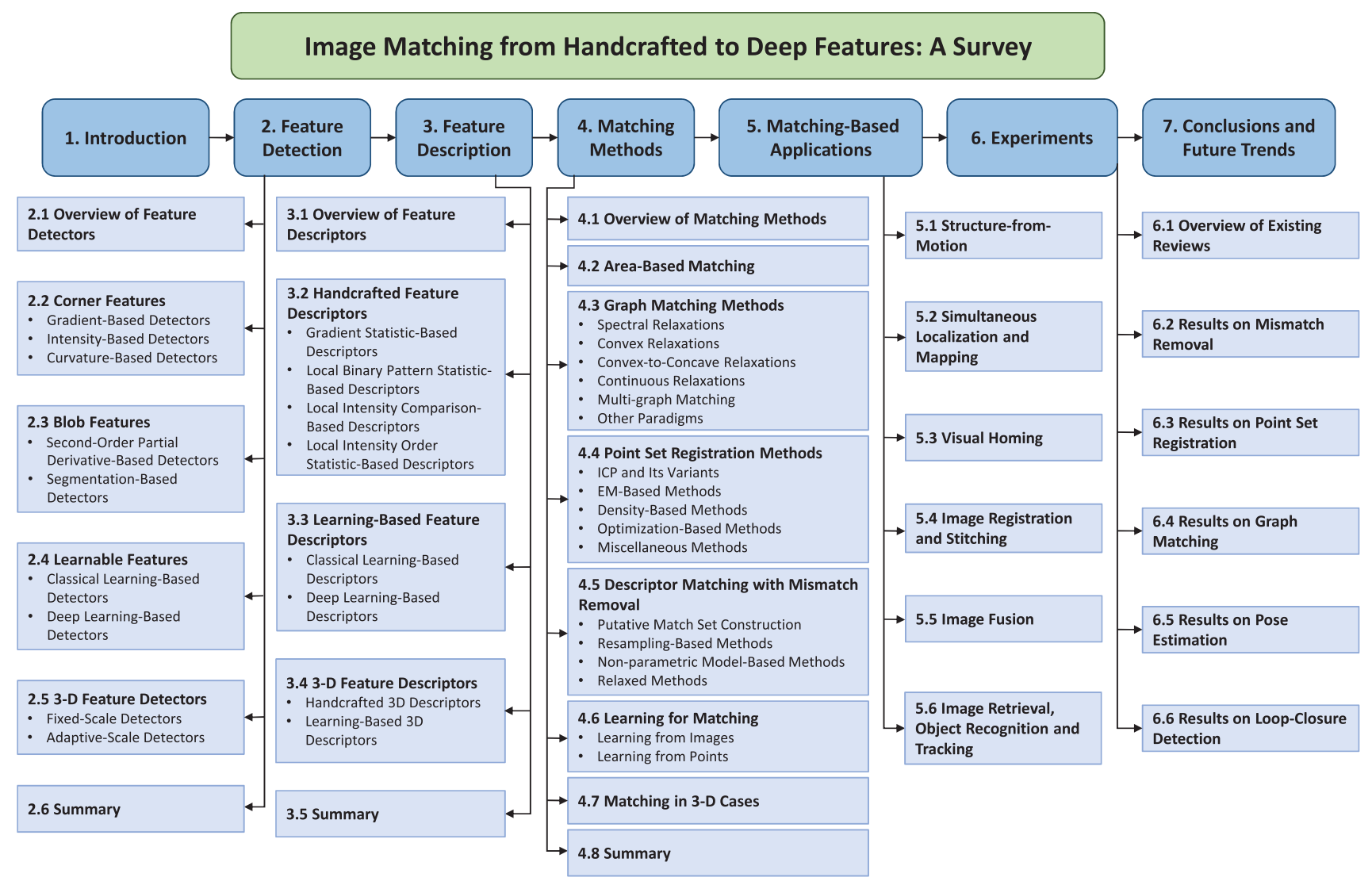

Fig. 1 Structure of this survey

Existing surveys are focused on different parts of image matching tasks and fail to cover the literature from the last decade. For instance, the early reviews (Zitova and Flusser 2003; Tuytelaars and Mikolajczyk 2008; Strecha et al. 2008; Aanæs et al. 2012; Heinly et al. 2012; Awrangjeb et al. 2012; Li et al. 2015) typically focus on handcrafted methods, which are not sufficient to provide a valuable reference for investigating CNN-based methods. Most recent reviews involve trainable techniques, but they merely cover a single part of image matching community, either focus on detectors (Huang et al. 2018; Lenc and Vedaldi 2014) or descriptors (Balntas et al. 2017; Schonberger et al. 2017) or specific matching tasks (Ferrante and Paragios 2017; Haskins et al. 2020; Yan et al. 2016b; Maiseli et al. 2017), and many others pay more attention on related applications (Fan et al. 2019; Guo et al. 2016; Zheng et al. 2018; Piasco et al. 2018). In this survey, we aim to provide an up-to-date and comprehensive summary and assessment of existing image matching methods, especially for the recently introduced learning-based methods. More importantly, we have provided a detailed evaluation and analysis for mainstream methods which are missing in existing literature.

This survey mainly focuses on feature-based matching, although patch matching, point set registration, and other related matching tasks are also reviewed. The overall organization is presented in Fig. 1; Sects. 2 and 3 describe the feature detection and description techniques respectively, from handcrafted methods to trainable ones. Patch matching is classified as a feature description domain, and 3-D point set features are also reviewed. In Sect. 4, we present different matching methods, including area-based image matching, pure point set registration, image descriptor similarity matching and mismatch removal, graph matching, and learning-based methods. Sections 5 and 6 respectively introduce the image matching-based visual applications and evaluation metrics, including the performance comparison. In Sect. 7, we conclude and discuss possible future developments.

\section{Feature Detection}

Early image features are annotated manually, which are still used in some low-quality image matching. With the development of computer vision and the requirement for auto-matching approaches, many feature detection methods have been introduced to extract stable and distinct features from images. 


\subsection{Overview of Feature Detectors}

Detected features represent specific semantic structures in an image or the real world and can be divided into corner feature (Moravec 1977; Harris et al. 1988; Smith and Brady 1997; Rosten and Drummond 2006; Rublee et al. 2011), blob feature (Lowe 2004; Bay et al. 2006; Agrawal et al. 2008; Yi et al. 2016), line/edge (Harris et al. 1988; Smith and Brady 1997; Canny 1987; Perona and Malik 1990), and morphological region feature (Matas et al. 2004; Mikolajczyk et al. 2005). However, the most popular features that are used for matching are the points (a.k.a. keypoints or interest points). The points are easy to extract and define with a simplified form compared with the line and region features, which can be roughly classified into corner and blob.

A good interest point must be easy to find and ideally fast to compute, as an interest point at a good location is crucial for further feature description and matching. To promote (i) matchability, (ii) the capability for subsequent applications, and (iii) matching efficiency and reduction of storage requirements, many required properties have been proposed for reliable feature extraction (Zitova and Flusser 2003; Tuytelaars and Mikolajczyk 2008), including repeatability, invariance, robustness and efficiency. The common idea for feature detection is to construct a feature response to distinguish salient point, line, and region from one another, along with flat and nondistinctive image areas. This idea can be subsequently classified into gradient-, intensity-, secondorder derivative-, contour curvature-, region segmentation-, and learning-based detectors. In the following, we provide a comprehensive introduction of feature detectors with these methods, focusing more on learning-based methods to guide researchers on how the traditional and trainable detectors work and give insights on their strengths and weaknesses.

\subsection{Corner Features}

A corner feature can for example be defined as the crossing point of two straight lines with the forms of "L", "T", "X", or a high curvature point of a contour. The common idea of corner detection is to compute a corner response and distinguish it from edge, flat, or other less distinctive image areas. Different strategies can be utilized for traditional corner searching, namely, gradient-, intensity-, and contour curvature-based. Refer to Zitova and Flusser (2003), Li et al. (2015), Tuytelaars and Mikolajczyk (2008) and Rosten et al. (2010) for details.

\subsubsection{Gradient-Based Detectors}

A gradient-based corner response prefers the use of the firstorder information in image to distinguish the corner feature. The earliest automatic corner detection method could be traced to Moravec detector (Moravec 1977), which first introduced the concept of "interest points" to define the distinct feature points, which are extracted based on the autocorrelation of the local intensity. This method calculates and searches the minimum intensity variation of each pixel from a shifted window in eight directions, and the interest point is detected if the minimum is superior to the given threshold.

However, the Moravec detector is not invariant to the direction or image rotation due to the discontinuous comparing directions and sizes. The famous Harris corner detector (Harris et al. 1988) was introduced to address the anisotropy and computation complexity problem. The goal of the Harris method is to find the directions of the fastest and lowest grey-value changes using a two-order moment matrix or an auto-correlation matrix; thus, it is invariant to orientation and illumination and has reliable repeatability and distinctiveness. Harris was further improved in Shi and Tomasi (1993) for better tracking performance by making the features more "spread out" and locating more accurately.

\subsubsection{Intensity-Based Detectors}

Several template- or intensity comparison-based corner detectors have been proposed by comparing the intensity of the surrounding pixels with that of the center pixel to simplify the image gradient computing. Due to their binary nature, they are widely used in many modern applications, particularly some with storage and real-time requirements.

The intensity-based corner detector, namely, smallest univalue segment assimilating nucleus (SUSAN) (Smith and Brady 1997), is based on the brightness similarity between the local radius region pixels and the nucleus. SUSAN can be implemented rapidly because it does not require gradient computation. Many analogous methods have been proposed based on the concept of brightness comparison, the most famous of which is the FAST detector (Trajković and Hedley 1998). FAST uses binary comparison with each pixel along a circle pattern against the central pixel and then determines more reliable corner features using a machine learning (i.e., ID3 tree Quinlan 1986) strategy, which is trained on a large number of similar scene images and can generate the best criteria for corner selection.

As an improvement of SUSAN, FAST is extremely efficient with high repeatability and is used more widely. To improve FAST without loss of efficiency, FAST-ER (Rosten et al. 2010) was introduced to enhance the repeatability by generalizing the detector based on further pixel intensity comparison centered on the nucleus. Another improvement is the AGAST (Mair et al. 2010), in which two more pixel brightness comparison criteria are defined, after which an optimal and specialized decision tree is trained in an extended configuration space, thus rendering the FAST detector more generic and adaptive. To combine the efficiency of FAST 
and the reliability of the Harris detector, Rublee et al. (2011) proposed an integrated feature detector and descriptor for matching called ORB. The ORB uses the Harris response to select a certain number of FAST corners as the final detected features. The gray-scale centroid of the local patch and the center pixel itself are formed as a vector to represent the main direction of the ORB feature, which helps calculate the similarity of the binary descriptor in ORB. Recently, a Sadder-like detector (Aldana-Iuit et al. 2016) has been proposed to extract interest points. In this detector, the saddle condition is verified efficiently by intensity comparisons on two concentric rings with certain geometric constraints. The Sadder detector can achieve higher repeatability and greater spread out than traditional methods even modern trainable ones (Komorowski et al. 2018).

\subsubsection{Curvature-Based Detectors}

Another strategy for corner feature extraction is based on detected high-level image structures, such as edges, contours, and salient regions. Corner features can be defined immediately as the midpoint/endpoint or sparse sampling from an edge or contour (Belongie et al. 2002). These are subsequently used for shape matching or point registration, especially for an image pair of less texture or binary type. The curvature-based strategy aims to extract the corner point with the maximum curvature searching based on the detected image curve-like edges. This strategy starts with an edge extraction and selection method, and the two subsequent steps are the curve smoothing and curvature estimation. The corners are finally determined by selecting the curvature extremum points. In general, an edge detector is often first in need for contour curvature-based corner detection.

In curve smoothing, the slope and curvature are difficult to evaluate due to the quantized position of a curve point. Noise and local deformation in a curve may also lead to a serious impact on the feature stability and distinctiveness. Therefore, smoothing methods should be implemented before or during the curvature calculation to make the curvature extremum points more distinct from other curve points. Two smoothing strategies, namely, direct and indirect methods, are generally utilized. A direct smoothing, such as Gaussian smoothing (Mokhtarian and Suomela 1998; Pinheiro and Ghanbari 2010), removes noise and may change curve locations to a certain extent. In comparison, in the indirect smoothing strategy, e.g., the region of support method or the chord-length-based method (Ramer 1972; Awrangjeb and Lu 2008), may preserve the curve point locations.

As for curvature estimation, for each point of the smoothed curve, a significance response measure is needed for corner searching, i.e., curvature. Curvature estimation methods are also generally classified as direct and indirect. The former is based on an algebraic or geometric estimation, such as cosine, local curvature, and tangential deflection (Mokhtarian and Suomela 1998; Rosenfeld and Weszka 1975; Pinheiro and Ghanbari 2010). The latter estimates the curvature in an indirect way and is often used as a significance measure, such as counting the number of curve points through several moving rectangles along the curve (Masood and Sarfraz 2007), using the perpendicular distances from the chord connecting the two endpoints of the curve to curve points (Ramer 1972), and other alternatives (Zhang et al. 2010, 2015). Compared with indirect estimation methods, the direct ones are more sensitive to noise and local variation due to the less neighboring point consideration.

Finally, corners can be determined with threshold strategy to remove false and indistinctive points (Mokhtarian and Suomela 1998; Awrangjeb and Lu 2008). Additional details can be obtained from a contour curvature-based corner survey (Awrangjeb et al. 2012). In addition and more recently, a multiscale segmentation-based corner detector, named MSFD (Mustafa et al. 2018), has been proposed for wide-baseline scene matching and reconstruction. Feature points in MSFD are detected at the intersection of the boundaries of three or more regions by using off-the-shelf segmentation methods. MSFD can generate rich and accurate corner features for wide-baseline image matching and high reconstruction performance.

The above-mentioned corner feature detectors are easily located in the contour or edge structures of an image (i.e., not such spread-out or uneven distribution), and are limited by the scale and affine transformation between two images. Among the three types of corner detection strategies, the gradientbased methods are able to locate more accurately, whereas the intensity-based methods show advantage for efficiency. The contour curvature-based methods require more computation but they are a better choice for processing textureless or binary images, such as infrared and medical images, because the image cue-based feature descriptors are unworkable for these types of images and the point-based descriptors are often coupled for the matching task (i.e., point set registration or shape matching). Please refer to Sects. 3 and 4 for details.

\subsection{Blob Features}

A blob feature is commonly indicated as a local closed region (e.g., with a regular shape of circle or ellipse), inside which the pixels are considered similar to one another and are distinct from the surrounding neighborhoods. The blob feature can be written in the form of $(x, y, \theta)$, with $(x, y)$ being the pixel coordinate of the feature location and $\theta$ indicating the blob shape information of the feature, including scale and/or affine. Numerous blob feature detectors have been introduced over the past decades, and they can be roughly classified into second-order partial derivative- and 
region segmentation-based detectors. Second-order partial derivative-based methods are based on the Laplacian scale selection and/or Hessian matrix calculation for affine invariant. While segmentation-based methods prefer to detect blob features by segmenting the morphological regions first, then estimate the affine information with ellipse fitting. Compared with corner features, blob features are more useful for visual applications with high precision requirement, because more image cues are utilized for feature identification and representation, thus enabling the blob features to be more accurate and robust to image transformation.

\subsubsection{Second-Order Partial Derivative-Based Detectors}

In methods based on second-order partial derivatives, the Laplacian of Gaussian (LoG) (Lindeberg 1998) is applied based on scale space theory. Here, the Laplace operator is first used for edge detection in accordance with the zero crossings in the second-order differential of an image, and the Gaussian convolution filtering is then applied as a preprocessing to reduce noise.

LoG can detect the local extremum point and the area with normalized response arising from the circular symmetry of the Gaussian kernel. Different standard deviations of the Gaussian function can detect the scale-invariant blobs in different scales by searching the extremum in the multiscale space as the final stable blob feature. The difference of Gaussians (DoG) (Lowe et al. 1999; Lowe 2004) filter can be used to approximate the LoG filter, and greatly speeds up the computations. Another classical blob feature detection strategy is based on the determinant of Hessian (DoH) (Mikolajczyk and Schmid 2001, 2004). This is more affine invariant because the eigenvalue and eigenvector of the second matrix can be applied to estimate and correct the affine region.

Interest point detection by using $\mathrm{DoG}, \mathrm{DoH}$, and both has been widely utilized in recent visual applications. The famous SIFT (Lowe et al. 1999; Lowe 2004) extracts keypoint as the local extrema in a DoG pyramid, filtered using the Hessian matrix of the local intensity values (the according description part will be reviewed in the next section). Mikolajczyk et al. combined the Harris and Hessian detectors with the Laplacian and Hessian matrices for scale and affine feature detection (Mikolajczyk and Schmid 2001, 2004), i.e., the Harris/Hessian-Laplacian/affine. SURF (Bay et al. 2006) accelerates the SIFT by approximating the Hessian matrixbased detector using Haar wavelet calculation, together with an integral image strategy, thus simplifying the construction of a second-order differential template.

Several SIFT- and SURF-based improvements, have been successively proposed for better property in subsequent applications. Such improvements include a fully affine invariant SIFT detector (ASIFT) (Morel and Yu 2009), a center- surround extremum (Agrawal et al. 2008) strategy feature detector with the Laplace calculation approximated by the proposed bilateral filtering to enhance the efficiency, and the efficient approximation of DoH with piecewise triangle filters in DARTs (Marimon et al. 2010). In addition, a cosinemodulated Gaussian filter is utilized in the SIFT-ER detector (Mainali et al. 2013) to obtain high feature detectability with minimum scale-space localization errors, in which the filterbank system has a highly accurate filter approximation without any image sub/upsampling. An edge foci-based blob detector (Zitnick and Ramnath 2011) has also been introduced for the matching task. In this detector, the edge foci is defined as the point in an image that is roughly equidistant from the closest edge with orientations perpendicular to this point.

Unlike the circle-like Gaussian response function, a nonlinear partial differential equation is applied in KAZA detector for blob feature searching with nonlinear diffusion filtering (Alcantarilla et al. 2012). An accelerated version called AKAZA (Alcantarilla and Solutions 2011) is implemented by embedding the fast explicit diffusion in a pyramidal framework to dramatically speedup feature detection in nonlinear scale spaces. However, it still suffers from high computation complexity. Another method is WADE (Salti et al. 2013), which implements nonlinear feature detection by a wave propagation function.

\subsubsection{Segmentation-Based Detectors}

The segmentation-based blob detectors begin with an irregular region segmentation based on constant pixel intensity or zero gradient. One of the most famous region segmentationbased blob feature is maximally stable extremal region (MSER) (Matas et al. 2004). It extracts regions that remain stable under a large range of intensity thresholding values. This approach does not need extra processing for scale estimation, and is robust to large viewpoint changes. The term "maximally stable" describes the threshold selection process, given that every extremal region is a connected component of a watershed image by thresholding. An extension to MSER was introduced in Kimmel et al. (2011) to exploit shape structure cues. Other improvements are based on the watershed regions of principal curvature images (Deng et al. 2007; Ferraz and Binefa 2012) or considered color information for a higher discrimination (Forssén 2007).

Similar to MSER, other segmentation-based features, such as intensity- and edge-based regions (Tuytelaars and Van Gool 2004), are also used for affine covariant region detection. However, feature detection of this type is of less use for feature matching, and it is gradually developed toward saliency detection and segmentation in computer vision. Specific method investigation and comprehensive reviews can be found in Mikolajczyk et al. (2005) and Li et al. (2015). 


\subsection{Learnable Features}

Over the recent years, data-driven learning-based methods have achieved significant progress in general visual pattern recognition tasks, and have also been applied to image feature detection. This pipeline can be roughly classified into the using of classical learning and deep learning.

\subsubsection{Classical Learning-Based Detectors}

Early from the past decade, classical learning-based methods, such as decision tree, support vector machine (SVM), and other classifiers by opposition to Deep Learning, have already been used in handcrafted keypoint detection (Trajković and Hedley 1998; Strecha et al. 2009; Hartmann et al. 2014; Richardson and Olson 2013). FAST (Trajković and Hedley 1998) detector was the first attempt to use traditional learning for reliable and matchable point identification, and similar strategies have been applied in many subsequent improvements (Mair et al. 2010; Rublee et al. 2011). Strecha et al. (2009) trained the Wald-Boost classifier to learn keypoints with high repeatability on pre-aligned training sets.

More recently, Hartmann et al. (2014) showed that it can be learnt from a structure-from-motion (SfM) pipeline to predict which candidate points are matchable, thus significantly reducing the number of interest points without losing excessive true matches. Meanwhile, Richardson and Olson (2013) reported that hand-designed detectors can be learned by random sampling in the space of convolutional filters and tried to find the optimal filter using a learning strategy over frequency-domain constraints. However, classical learning has only been used for reliable feature selection through classifier learning, rather than the extraction of interest features directly from raw images until the emergence of deep learning.

\subsubsection{Deep Learning-Based Detectors}

Inspired by the handcrafted feature detectors, a general solution for CNN-based detection is to construct response maps to search the interest points in a supervised (Yi et al. 2016; Verdie et al. 2015; Zhang et al. 2017b), self-supervised (Zhang and Rusinkiewicz 2018; DeTone et al. 2018), or unsupervised manner (Lenc and Vedaldi 2016; Savinov et al. 2017; Ono et al. 2018; Georgakis et al. 2018; Barroso-Laguna et al. 2019). The task is often converted into a regression problem that can be trained in a differentiable way under the transformation and imaging condition invariance constraints. Supervised methods have shown the benefits of using anchors (e.g., obtained from SIFT method) to guide their training, but the performance could be largely restricted by the method of anchor construction, because the anchor itself is intrinsically difficult to reasonably define and may pre- vent the network from proposing new keypoints in case no anchor exists in the proximity (Barroso-Laguna et al. 2019). Self-supervised and unsupervised methods train detectors without any human annotations, and only the geometric constraints between two images are required for optimization guidance; a simple human aid is sometimes asked for pretraining (DeTone et al. 2018). In addition, many methods integrate feature detection into the entire matching pipeline by jointly training with feature description and matching (Yi et al. 2016; DeTone et al. 2018; Ono et al. 2018; Shen et al. 2019; Dusmanu et al. 2019; Choy et al. 2016; Rocco et al. 2018; Dusmanu et al. 2019; Revaud et al. 2019), which can enhance the final matching performance and optimize the entire procedure in an end-to-end manner.

For instance, TILDE (Verdie et al. 2015) trains multiple piecewise linear regression models to detect repeatable keypoints under drastic imaging changes of weather and lighting conditions. First, it identifies good keypoint candidates in multiple training images taken from the same viewpoints using DoG for training set collection, and then trains a general regressor to predict a score map, whose maxima after non-maximum suppression (NMS) can then be regarded as the desired interest points.

DetNet (Lenc and Vedaldi 2016) is the first fully general formulation for learning local covariant features; it casts the detection task as a regression problem and then derives a covariance constraint to automatically learn stable anchors for local feature detection under geometric transformations. Meanwhile, Quad-net (Savinov et al. 2017) realizes keypoint detection under transformation-invariant quantile ranking with a single real-valued response function, enabling it to learn the detector completely from scratch by optimizing for a repeatable ranking. A similar detector in Zhang and Rusinkiewicz (2018) combines this "ranking" loss with a "peakedness" loss and produces a more repeatable detector.

Zhang et al. (2017b) proposed TCDET detector by defining a novel formulation based on the new concepts of "standard patch" and "canonical feature" to place equal focus on discriminativeness and covariant constraint. The proposed detector can detect discriminative and repeatable features under diverse image transformations. Key.Net (BarrosoLaguna et al. 2019) combines handcrafted and learned CNN filters within a shallow multiscale architecture and proposes a light/efficient trainable detector. The handcrafted filters provide anchor structures for localizing, scoring, and ranking repeatable features that are fed to learned filters. $\mathrm{CNN}$ is used to represent the scale space by detecting keypoints at different levels; the loss function is defined to detect robust feature points from different scales and maximize the repeatability score. The affine region-based interest point is also learned using CNNs in Mishkin et al. (2017, 2018).

The methods of integrating a detector into a matching pipeline are similar to those solely designed for detection 
reviewed above. The main difference may lie in the way of training, and the core challenge is to make the entire process differentiable. For example, Yi et al. (2016) attempted to train a detector, an orientation estimator, and a descriptor jointly based on inputting four patches. Their proposed LIFT can be regarded as a trainable version of SIFT and requires supervision from the SfM system for determining the feature anchor. The training procedure is conducted individually from descriptor to detector and can use the learned results to guide the detector training, thus promoting detectability. Unlike LIFT, SuperPoint (DeTone et al. 2018) introduces a fully convolutional model by inputting full-sized images and jointly computing pixel-level interest point locations and associated descriptors in one forward pass; a synthetic dataset is constructed for pseudo-ground truth generation and pretraining, and the homography adaption module enables it to achieve self-supervised training while promoting detection repeatability.

LF-Net (Ono et al. 2018) confines the end-to-end pipeline to one branch to optimize the entire procedure in a differentiable way; it also uses a fully convolutional network operating on full-sized images to generate a rich feature score map, which can then be used to extract keypoint locations and the feature attributes, such as scale and orientation; simultaneously, it performs a differentiable form of NMS, namely, softargmax, for subpixel location and increasing the accuracy and saliency of keypoint. Similar to LF-Net, RF-Net (Shen et al. 2019) selects high-response pixels as keypoints on multiscales, but the response maps are constructed by receptive feature maps. Bhowmik et al. (2020) indicated that increased accuracy for these low-level matching scores does not necessarily translate to better performance in high-level vision tasks, thus they embedded the feature detector in a complete vision pipeline, where the learnable parameters are trained in an end-to-end manner. The authors overcome the discrete nature of keypoint selection and descriptor matching using principles from reinforcement learning. Luo et al. (2020) proposed ASFeat to explore local shape information of feature points and enhance the accuracy of points detection, by jointly learning local feature detectors and descriptors. Another detection-related learning-based method is to estimate the orientation (Moo Yi et al. 2016), while the spatial transformation network (STN) (Jaderberg et al. 2015) could also be a great reference in deep learning-based detectors for rotation invariance (Yi et al. 2016; Ono et al. 2018).

Unlike local feature descriptors, there is little review on salient feature detectors, particularly for the recent CNNbased techniques. To our best knowledge, the most recent survey (Lenc and Vedaldi 2014) focuses on local feature detection. It introduces the basic idea of several wellknown methods from handcrafted detectors to accelerated and learned ones.

\subsection{3-D Feature Detectors}

Dedicated on 3-D keypoint detectors, Tombari et al. (2013) provided an excellent survey on the state-of-the-art methods and a detailed evaluation of their performances. In brief, the existing methods were divided into two categories, fixed-scale detectors and adaptive-scale detectors. In both categories, keypoints are selected as local extrema of a predefined saliency measurement. The difference lies in the involvement of the scale characteristic, which defines the support for the subsequent description stage. The fixedscale detectors tend to search keypoints at a specific scale level, which is given as prior information. The adaptive-scale detectors either extend the scale concept for 2-D images by adopting a scale space defined on the surface or implement the traditional scale-space analysis by embedding 3-D data onto a 2-D plane.

\subsubsection{Fixed-Scale Detectors}

Chen and Bhanu (2007) introduced the local surface patch (LSP) method. The saliency of a point in LSP is measured by its shape index (Dorai and Jain 1997), as defined by the principal curvatures at the point. Zhong (2009) introduced the intrinsic shape signature (ISS) method, in which saliency is derived from the eigenvalue decomposition of the scatter matrix of the support region. In this approach, the ratio of eigenvalues is used to prune some points, and the final saliency is determined by the eigenvector. In this way, points with large variations along each principal direction are identified. Analogous to ISS, Mian et al. (2010) also utilized the scatter matrix to prune nondistinctive points but with a different curvature-based saliency measurement. Sun et al. (2009) presented the heat kernel signature (HKS) method, based on the properties of the heat diffusion process on a shape. In this method, the saliency measurement is defined by the restriction of the heat kernel to the temporal domain. The heat kernel is uniquely determined by the underlying manifold, which makes HKS a compact characterization of the shape.

\subsubsection{Adaptive-Scale Detectors}

It is desirable to adaptively fit with the scale in detection. For this purpose, Unnikrishnan and Hebert (2008) proposed a Laplace-Beltrami scale space by computing the designed function on the increasing support around each point. This function is defined by a novel operator that reflects the local mean curvature of the underlying shape and provides the saliency information. Zaharescu et al. (2009) presented the MeshDoG method, which is analogous to the DoG operator in the 2-D case (Lowe 2004); nonetheless, the operator is computed on a scalar function defined on the manifold. The 
output of the DoG operator represents the saliency for keypoints detection. Castellani et al. (2008) also built scale space using the DoG operator but directly on the 3-D mesh. Mian et al. (2010) proposed an automatic scale selection technique for extracting scale invariant features. The scale space is built by increasing the support size, and automatic scale selection at each keypoint is performed by using NMS along scale. The disadvantage of sensitivity to scale of HKS was addressed by Bronstein and Kokkinos (2010), who used Fourier transform magnitude to extract a scale-invariant quantity from the HKS without the need to perform scale selection. Sipiran and Bustos (2011) extended the well-known Harris operator (1988) into 3-D data with an adaptive-scale determination technique. Readers are referred to Tombari et al. (2013) for further discussion on other adaptive-scale detectors. Salti et al. (2015) devised a learning-based 3-D keypoint detector, whereby the keypoint detection problem was cast as a binary classification problem, to determine whose support can be correctly matched by a predefined 3-D descriptor.

\subsection{Summary}

The basic idea of feature detectors is to distinguish the interest feature from others through the response value, thus leading to the solutions of two problems: (i) how to define discriminant patterns in an image, and (ii) how to repeatedly detect the salient feature under different image conditions and image qualities (Zhang et al. 2017b). Along with the development of these detectors, the main improvements and common strategies are related to four aspects, i.e., feature response type and improvements on efficiency, robustness, and accuracy, which lead to an increase in the matchability of detected features and the improved performance of their subsequent applications.

For traditional methods, using more image cues can result in better robustness and repeatability, but usually requires more computational cost. In addition to using low-order feature detectors, several strategies, such as approximate and pre-compute, are designed to largely speed up the computation and maintain the matchability. To ensure the robustness, scale and affine information estimation is usually required when searching stable features. While for accuracy enhancement, a local extremal searching for subpixel accuracy and NMS strategy in pixel and scale space to avoid features locally gathered, are two popular choices in traditional pipelines.

As for learning-based detectors, repeatable and salient keypoints can be extracted based on high-level cues captured by CNNs, except for intensity, gradient, or second-order derivative. While the efficiency would largely depend on the network structure, and early deep learning methods are often time-consuming. Methods proposed recently, such as SuperPoint and Key.Net, have already achieved good imple- mentation in real time while maintaining state-of-the-art performance. Multiscale sampling or changed receptive field would make these deep learning-based detectors invariant to scale, where the scale or rotation information is directly estimated in networks. They can achieve promising results, because the deep learning techniques can easily distinguish the same structures, despite the fact that images suffer from apparent variance and geometrical transformation. The accuracy can be optimized directly in the loss function of the learning-based methods, and the differentiable form of NMS is often used for subpixel accuracy location and repeatability enhancement.

\section{Feature Description}

Once discriminative interest points are detected from raw images, a local patch descriptor is required to be coupled for each feature in order to establish feature correspondence correctly and efficiently across two or more images. In other words, the feature descriptors are commonly used to transform the original local information around the interest point into a stable and discriminative form, usually as a highdimensional vector, so that two corresponding features are as close as possible in the descriptor space, and two noncorresponding features are as far as possible.

\subsection{Overview of Feature Descriptors}

The processing procedure of feature description can be divided into three steps: local low-level feature extraction, spatial pooling, and feature normalization (Lowe 2004; Rublee et al. 2011; Brown et al. 2010). First, the low-level information of a local image region has to be extracted. This information consists of pixel intensity and gradient or is obtained from a series of steerable filters. Subsequently, the local patch is divided into several parts and the local information is pooled in each part, then concatenate them by using pooling methods, such as rectangular gridding (Lowe 2004), polar gridding (Mikolajczyk and Schmid 2005), Gaussian sampling (Tola et al. 2010), and others (Rublee et al. 2011); the joint feature representation is transformed into a more discriminative one that may preserve significant information in a simplified form for better matching performance. Finally, a descriptor is obtained from the normalized results of the pooled local information, which aims to map the aggregated results into a long vector of either floating-point or binary values for easily evaluating the similarity between image features.

Similar to feature detectors, existing descriptors are proposed and improved to become highly robust, efficient, and discriminant for addressing image matching problems. Estimating a good size and orientation for a cropped image 
patch is core problems in the task of feature description and matching. By correctly identifying the size and orientation, the matching methods can be robust and invariant to global and/or local deformations, such as rotation and scaling. The original intention of feature description is focused on discrimination enhancement compared with direct similarity measurement using raw image information. Numerous well-designed descriptors can improve the discrimination and matching performance, by using pooling parameter optimization, sampling rule design, or the use of machine learning and deep learning techniques.

Feature description has drawn increasing attention. Descriptors can be regarded as distinguishable and robust representations for given images and are widely used not only in image matching but also in image coding for image retrieval, face recognition, and other tasks that are based on image similarity measurements. However, direct similarity measurements for two image patches using raw image information will be regarded as an area-based image matching method, which will be reviewed in the next section. As for image patch-based feature descriptors, we will review the traditional ones, i.e., floating and binary descriptors, in terms of their data types. A new subsection will be added for the recent data-driven methods, including classical machine learning- and emerging deep learning-based methods. We will comprehensively review handcrafted and learning-based feature description methods and show the connections among these methods to provide useful instructions for the readers toward their further research, especially for developing better description approaches using deep learning/CNN techniques. In addition, we will also review the 3-D feature descriptors, where features are typically obtained from point data without any image pixel information but with spatial position relationships (e.g., 3-D point cloud registration).

\subsection{Handcrafted Feature Descriptors}

Handcrafted feature descriptors often depend on expert priori knowledge, which are still widely used in many visual applications. Following the construction procedure of a traditional local descriptor, the first step is to extract low-level information, which can be briefly classified into image gradient and intensity. Subsequently, the commonly used pooling and normalizing strategies, such as statistic and comparison, are applied to generate long and simple vectors for discriminative description with respect to the data type (float or binary). Therefore, handcrafted descriptors mostly rely on the knowledge of their authors, and description strategies can be classified into gradient statistic-, local binary pattern statistic-, local intensity comparison- and local intensity order statistic-based methods.

\subsubsection{Gradient Statistic-Based Descriptors}

Gradient statistic methods are often used to form float type descriptors such as the histogram of oriented gradients (HOG) (Dalal and Triggs 2005) as introduced in SIFT (Lowe et al. 1999; Lowe 2004) and its improvement versions (Bay et al. 2006; Morel and Yu 2009; Dong and Soatto 2015; Tola et al. 2010), and they are still widely used in several modern visual tasks. In SIFT, feature scale and orientation are respectively determined by DoG computation and the largest bin in a histogram of gradient orientation from a local circular region around the detected keypoint, thus achieving scale and rotation invariance. In the description stage, the local region of detected feature is first rectangularly divided into $4 \times 4$ non-overlapping grids based on the normalized scale and rotation, then a histogram of gradient orientation with 8 bins is conducted in each cell and embedded into a 128dimensional float vector as the SIFT descriptor.

Another representative descriptor, namely, SURF (Bay et al. 2006), can accelerate the SIFT operator by using the responses of Haar wavelets to approximate gradient computation; integral images are also applied to avoid repeated computation in Haar wavelet responses, enabling more efficient computation than SIFT. Other improvements based on these two typically focus on discrimination, efficiency, robustness, and coping with specific image data or tasks. For instance, CSIFT (Abdel-Hakim and Farag 2006) uses additional color information to enhance the discrimination, and ASIFT (Morel and Yu 2009) simulates all image views obtainable by varying the two camera axis orientation parameters for fully affine invariance. Mikolajczyk and Schmid (2005) use a polar division and histogram statistics of gradient orientations. SIFT-rank (Toews and Wells 2009) has been proposed to investigate ordinal image description based on off-the-shelf SIFT for invariant feature correspondence. A Weber's law-based method (WLD) (Chen et al. 2009) has been studied to compute a histogram by encoding differential excitations and orientations at certain locations.

Arandjelović and Zisserman (2012) used a square root (Hellinger) kernel instead of the standard Euclidean distance measurement to transform the original SIFT space to the RootSIFT space and yielded superior performance without increasing processing or storage requirements. Dong and Soatto (2015) modified SIFT by pooling the gradient orientation across different domain sizes and proposed DSP-SIFT descriptor. Another efficient dense descriptor for wide-baseline stereo based on SIFT, namely, DAISY (Tola et al. 2010), uses a log-polar grid arrangement and Gaussian pooling strategy to approximate the histograms of gradient orientations. Inspired by DAISY, DARTs (Marimon et al. 2010) can efficiently compute scale space and reuse it for descriptors, thus resulting in high efficiency. Several handcrafted float-type descriptors have also been proposed 
recently and shown promising performance; for example, the pattern of local gravitational force local descriptor (Bhattacharjee and Roy 2019) is inspired from the law of universal gravitation and can be regarded as a combination of force magnitude and angle.

\subsubsection{Local Binary Pattern Statistic-Based Descriptors}

Different from SIFT-like approaches, several intensity statisticbased methods, which are inspired by the local binary pattern (LBP) (Ojala et al. 2002), have been proposed in the past decades. LBP has properties that favor its usage in interest region description, such as tolerance against illumination change and computational simplicity. The drawbacks are that the operator produces a rather long histogram and is insignificantly robust in flat image areas. Center-symmetric LBP (CS-LBP) (Heikkilä et al. 2009) (using SVM for classifier training) is a modified version of LBP combining the strengths of SIFT and LBP to address the flat area problem. Specifically, CS-LBP uses a SIFT-like grid and replaces the gradient information with an LBP-based feature. To address the noise, center-symmetric local ternary pattern (CS-LTP) (Gupta et al. 2010) suggests the use of a histogram of relative orders in patch and a histogram of LBP codes, such as histogram of relative intensities. The two CS-based methods are designed to be more robust to Gaussian noise than previously considered descriptors. RLBP (Chen et al. 2013) improves the robustness of LBP by changing the coding bit; a completed modeling of the LBP operator and an associated completed LBP scheme (Guo et al. 2010) have been developed for texture classification. LBP-like methods are widely used in texture representation and face recognition community, and additional details can be found in the review literature (Huang et al. 2011).

\subsubsection{Local Intensity Comparison-Based Descriptors}

Another form of descriptors is based on the comparison of local intensities, which is also called binary descriptors and the core challenge is the selection rule for comparison. Because of their limited distinctiveness, these methods are mostly limited to short-baseline matching. Calonder et al. (2010) proposed the BRIEF descriptor built by concatenation of the results of a binary test of intensities for several random point pairs in image patch. Rublee et al. (2011) proposed rotated BRIEF combined with oriented FAST corners and selected robust binary tests using an machine learning strategy in their ORB algorithm to alleviate the limitations in rotation and scale change. Leutenegger et al. (2011) developed the BRISK method using a concentric circle sampling strategy with increasing radius. Inspired by the retina structure, Alahi et al. (2012) proposed the FREAK descriptor by comparing image intensities over a retinal sampling pattern for fast computing and matching with low memory cost while remaining robust to scale, rotation, and noise. Handcrafted binary descriptors and classical machine learning techniques are also widely studied and these shall be introduced in the learning-based subsection.

\subsubsection{Local Intensity Order Statistic-Based Descriptors}

Thus far, many methods have been devised using orders of pixel values rather than raw intensities, achieving more promising performance (Tang et al. 2009; Toews and Wells 2009). Pooling by intensity orders is invariant to rotation and monotonic intensity changes and also encodes ordinal information into descriptor; the intensity order-pooling scheme may enable the descriptors to be rotation-invariant without estimation of a reference orientation as SIFT, which appears as a major error source for most existing methods. To solve this problem, Tang et al. proposed the ordinal spatial intensity distribution (Tang et al. 2009) method, which normalizes captured texture information and structure information using an ordinal and spatial intensity histogram; the proposed method is invariant to any monotonically increasing brightness changes.

Fan et al. (2011) pooled local features based on their gradient and intensity orders in multiple support regions and proposed the multi-support region order-based gradient histogram and the multi-support region rotation and intensity monotonic invariant descriptor methods. A similar strategy was used in LIOP (Wang et al. 2011, 2015), to encode the local ordinal information of each pixel. In that work, the overall ordinal information was used to divide the local patch into subregions, which were used to accumulate LIOP. LIOP was further improved into OIOP/MIOP (Wang et al. 2015), which can then encode overall ordinal information for noise and distortion robustness. They also proposed a learning-based quantization to improve its distinctiveness.

\subsection{Learning-Based Feature Descriptors}

Handcrafted descriptors, as reviewed above, require expertise to design and may disregard useful patterns hidden in the data. This requirement has prompted the investigations on learning-based descriptors, which have recently become dominantly popular due to their data-driven property and promising performance. In the following, we will discuss a group of classical learning-based descriptors introduced before the deep learning era.

\subsubsection{Classical Learning-Based Descriptors}

The learning-based descriptors can be traced back to PCASIFT (Ke et al. 2004), in which principal component analysis (PCA) is used to form a robust and compact descriptor by 
reducing the dimensionality of a vector made of the local image gradients. Cai et al. (2010) investigated the use of linear discriminant projections to reduce dimensionality and improve the discriminability of local descriptors. Brown et al. (2010) introduced a learning framework with a set of building blocks for constructing descriptors by using Powell minimization and linear discriminant analysis (LDA) technique to find the optimal parameters. Simonyan et al. (2014) presented a novel formulation to represent the spatial pooling and dimensionality reduction in descriptor learning as convex optimization problems based on Brown's work (Brown et al. 2010). Meanwhile, Trzcinski et al. (2012, 2014) applied the boosting trick to learn boosted, complex non-linear local visual feature representations from multiple gradient-based weak learners.

Apart from the above-mentioned float-valued descriptors, binary descriptors are also of great interest in classical descriptor learning due to their beneficial properties, such as low storage requirements and high matching speed. A natural way to obtain binary descriptors is to learn it from the provided float-valued descriptors. This task is conventionally achieved by the hashing methods, thus suggesting that compact representations of high-dimensional data should be learned while maintaining their similarity in the new space. Locality sensitive hashing (LSH) (Gionis et al. 1999) is arguably a popular unsupervised hashing method. This method generates embeddings via random projections and has been used for many large-scale search tasks. Some variants of LSH include kernelized LSH (Kulis and Grauman 2009), spectral hashing (Weiss et al. 2009), semantic hashing (Salakhutdinov and Hinton 2009) and p-stable distributionbased LSH (Datar et al. 2004). These variants are unsupervised by design.

Supervised hashing methods have also been extensively investigated, where different machine learning strategies have been proposed to learn feature spaces tailored to specific tasks. In this case, a plethora of methods have been proposed (Kulis and Darrell 2009; Wang et al. 2010; Strecha et al. 2012; Liu et al. 2012a; Norouzi and Blei 2011; Gong et al. 2013; Shakhnarovich 2005), among which image matching is considered an important experimental validation task. For example, the LDA technique is utilized in Strecha et al. (2012) to aid hashing. Semi-supervised sequential learning algorithms are proposed in Liu et al. (2012a) and Wang et al. (2010) to find discriminative projections. Minimal loss hashing (Norouzi and Blei 2011) provided a new formulation to learn binary hash functions on the basis of structural SVMs with latent variables. Gong et al. (2012) proposed searching a rotation of zero-centered data to minimize the quantization error of mapping the descriptor to the vertices of a zerocentered binary hypercube.

Trzcinski and Lepetit (2012) and Trzcinski et al. (2017) reported that a straightforward way of developing binary descriptors is to directly learn representations from image patches. In Trzcinski and Lepetit (2012), they proposed to project image patches to a discriminant subspace by using a linear combination of a few simple filters and then threshold their coordinates for creating the compact binary descriptor. The success of descriptors (e.g., SIFT) during image matching indicates that non-linear filters, such as gradient response, are more suitable than linear ones. Trzcinski et al. (2017) proposed to learn a hash function of the same form as an AdaBoost strong classifier, i.e. the sign of a linear combination of nonlinear weak learners, for each descriptor bit. This work is more general and powerful than Trzcinski and Lepetit (2012), which is based on simple thresholded linear projections. Trzcinski et al. (2017) proposed to generate binary descriptors that are independently adapted per patch. This objective is achieved by inter- and intra-class online optimization for descriptors.

\subsubsection{Deep Learning-Based Descriptors}

Descriptors using deep techniques are usually formulated as a supervised learning problem. The objective is to learn a representation that can enable the two matched features to be as close as possible while the unmatched ones are far apart in the measuring space (Schonberger et al. 2017). Descriptor learning is often conducted with cropped local patches centered on the detected keypoints; thus, it is also known as patch matching. In general, existing methods consist of two forms, namely, metric learning (Weinberger and Saul 2009; Zagoruyko and Komodakis 2015; Han et al. 2015; Kedem et al. 2012; Wang et al. 2017; Weinberger and Saul 2009) and descriptor learning (Simo-Serra et al. 2015; Balntas et al. 2016a, 2017; Zhang et al. 2017c; Mishchuk et al. 2017; Wei et al. 2018; He et al. 2018; Tian et al. 2019; Luo et al. 2019), according to the output of deep learning-based descriptors. These two forms are often jointly trained. Specifically, metric learning methods often learn a discriminative metric for similarity measurement with raw patches or generated descriptors as inputs. By contrast, descriptor learning tends to generate the descriptor representation from raw images or patches. Such a process requires a measurement method, such as L2 distance or trained metric network, for similarity evaluation. In contrast with single metric learning, the use of CNNs to generate description vectors is more flexible and may save time by avoiding repeated computation when a large number of candidate patches are available for correspondence search. Deep learning has achieved satisfying performance in feature description due to its strong ability in information extraction and representation.

Descriptors with deep learning techniques can be regarded as an extension of those based on classical learning (Schonberger et al. 2017). For instance, the Siamese structure in Chopra et al. (2005) and the commonly used loss func- 
tions, such as hinge, Siamese, triplet, ranking, and contrastive losses, have been borrowed and modified in recent deep methods. Specifically, Zagoruyko and Komodakis (2015) proposed their DeepCompare and demonstrated the mechanism by which to directly learn from raw image pixels with a general patch similarity function. In such scenario, various Siamese-type CNN models are applied to encode the similarity function. These models are then trained to identify the positive and negative image patch pairs. The attempted different network structures include Siamese with shared or unshared weights and central-surround form. MatchNet (Han et al. 2015) is proposed to simultaneously learn the descriptor and metric. Such a technique is implemented by cascading a Siamese-like description network and fully convolutional decision network. The task is converted into a classification problem under a cross-entropy loss. DeepDesc (Simo-Serra et al. 2015) uses CNNs to learn discriminant patch representations together with L2 distance measuring. In particular, it trains a Siamese network with pairs of positive and negative patches by minimizing the pairwise hinge loss, and the proposed hard negative mining strategy has alleviated the unbalanced positive and negative samples. Consequently, the description performance is siginificantly enhanced. Wang et al. (2014) proposed a novel deep ranking model to learn fine-grained image similarity. The model employs a triplet-based hinge loss and ranking function to characterize fine-grained image similarity relationships. A multiscale neural network architecture is utilized to capture the global visual properties and image semantics.

Kumar et al. (2016) first used the global loss to enlarge the distance margin between positive and negative patch pairs. It is implemented through triplet and Siamese networks trained with a combination of triplet and global losses. TFeat (Balntas et al. 2016b) proposes to utilize triplets of training samples for CNN-based patch description and matching. It is implemented with shallow convolutional networks and fast hard negative mining strategy. In L2Net (Tian et al. 2017), Tian et al. applied a progressive sampling strategy to optimize the relative distance-based loss function in the Euclidean space. The authors of that work considered the intermediate feature map and compactness of descriptor to achieve better performance. HardNet (Mishchuk et al. 2017) achieves better improvement than L2Net by using a simple hinge triplet loss with the "hardest-within-batch" mining. PN-Net (Balntas et al. 2016a) uses ideas introduced in the field of distance metric learning and online boosting by simultaneously training with positive and negative constraints. The proposed SoftPN loss function exhibits faster convergence and lower error than hinge loss or SoftMax ratio (Wang et al. 2014; Zagoruyko and Komodakis 2015). Zhang et al. (2017c) trained their networks by using their proposed global orthogonal regularization together with triplet loss for encouraging the descriptor to be sufficiently "spread out". It was carried out to fully utilize the descriptor space.

Descriptor learning based on average precision attention (He et al. 2018), introduces a general-purpose learning to rank formulation. This approach is defined to a constraint wherein the true matches should be ranked above all false path matches and is optimized on the basis of the binary and real-value local feature descriptors. BinGAN (Zieba et al. 2018) proposes a regularization method for generative adversarial networks (Goodfellow et al. 2014) to learn discriminative yet compact binary representations of image patches. In comparison, other methods focused on binary descriptor learning are proposed in Erin Liong et al. (2015), Lin et al. (2016a) and Duan et al. (2017). Except for loss function, network structure, regularization and hard negative mining, Wei et al. (2018) learned a discriminative deep descriptor by using kernelized subspace pooling. Tian et al. (2019) used second-order similarity in their SOSNet. In ContextDesc, a more recent method, Luo et al. (2019) combined the local patch similarity constraint with the spatial geometrical constraint of interest point to train their networks, which largely improves the matching performance. ${ }^{1}$

As mentioned in the CNN-based detectors, an increasing number of end-to-end learning methods integrate the feature description together with the detectors into the complete matching pipeline. These methods are similar to those that have been singly designed for the description reviewed above. The main difference may lie on the way of training and the design of the entire network structure. The core challenge is to make the whole process differentiable and trainable. For example, LIFT (Yi et al. 2016) attempts to simultaneously implement keypoint detection, orientation estimation, and feature description, by end-to-end CNN networks.

SuperPoint (DeTone et al. 2018) proposes a self-supervised framework for training interest point detectors and descriptors for multiple view geometrical problems. The fully convolutional model operates on full-sized images and jointly computes pixel-level interest point locations and associated descriptors, which is in contrast with path-based networks. LF-Net (Ono et al. 2018) devises a two-branch setup and creates virtual target responses iteratively to allow training from scratch without handcrafted priors. This technique realizes feature map generation, scale-invariant keypoint detection using top K selection and NMS, orientation estimation, and descriptor extraction. In LF-Net, the target function includes image level loss (satisfying additional constraints among image pairs, depth map, and essential matrix), patch-wise loss (learning keypoints that are good for matching and involves the orientation and scale component geometric consistency), and triplet loss for descriptor learning.

\footnotetext{
${ }^{1}$ https://image-matching-workshop.github.io/leaderboard/.
} 
Subsequently, RF-Net (Shen et al. 2019) creates an endto-end trainable matching framework that is modified from the LF-Net structure. First, the constructed receptive feature maps lead to effective keypoint detection. Second, a general loss function term, that is, neighbor mask, facilitates training patch selection to enhance the stability in descriptor training. D2-Net (Dusmanu et al. 2019) uses a single CNN to play a dual role: simultaneously achieving a dense feature descriptor and a feature detector. In Bhowmik et al. (2020), a keypoint selection and descriptor matching are optimized under high-level vision tasks by using principles from reinforcement learning. In addition, Li et al. (2020) introduced dual-resolution correspondence networks to obtain pixelwise correspondences in coarse-to-fine manner by extracting different resolution feature maps.

Except for feature matching for the same target or scene, semantic matching for images that are captured from similar targets/scenes has also been studied using CNNs and distinct promotion has been achieved. The semantic matching problem may pose a challenge for handcrafted methods due to the required understanding of semantic similarity. To this end, UCN (Choy et al. 2016) uses deep metric learning to directly learn a feature space that preserves either geometric or semantic similarity. The use of such an approach also helps generate dense and accurate correspondences for either geometric or semantic correspondence tasks. Specifically, UCN implements a fully convolutional architecture with a correspondence contrastive loss for fast training and testing, and proposes a convolutional spatial transformer for local patch normalization. NCN (Rocco et al. 2018) develops an end-to-end trainable CNN architecture based on the classic idea of disambiguating feature matching by using semi-local constraints to find reliable dense correspondences between a pair of images. This framework identifies sets of spatially consistent matches by analyzing the neighboring consensus patterns for a global geometric model. The model can be efficiently trained via weak supervision without any manual annotations of point correspondences. This type of framework can be applied for both category-level and instance-level matching tasks, and other similar methods are presented in Han et al. (2017), Plötz and Roth (2018), Chen et al. (2018), Laskar and Kannala (2018), Kim et al. (2018, 2020), Ufer and Ommer (2017) and Wang et al. (2018).

\subsection{3-D Feature Descriptors}

Extensive studies on 3-D feature descriptors have been conducted. As previously mentioned, many researchers have turned their attention to deep learning paradigm due to its revolutionary success in numerous different areas. This fact motivates us to categorize modern descriptors into two groups, i.e. handcrafted and learning-based ones. Guo et al. (2016) presented a comprehensive performance evaluation of conventional handcrafted 3-D feature descriptors, while the learning-based methods are left out. In the following section, we provide a brief introduction of the state-of-the-art handcrafted descriptors and the learning-based ones.

\subsubsection{Handcrafted 3-D Descriptors}

Guo et al. (2016) divided the handcrafted descriptors into spatial distribution histogram- and geometric attribute histogram-based descriptors, with the former representing the local feature by histograms that encode spatial distributions of the points in the support region. In general, the local reference frame/axis is constructed for each keypoint. Accordingly, the 3-D support region is partitioned into bins to form a histogram. The values of each bin are calculated by accumulating the spatial distribution measurements. Some representative work include spin image (Johnson and Hebert 1999), 3-D shape context (Frome et al. 2004), unique shape context (Tombari et al. 2010a), rotational projection statistics (Guo et al. 2013) and tri-spin-image (Guo et al. 2015). The spatial distribution histogram descriptors represent the local features by generating histograms from the statistics of geometric attributes (e.g., normals, curvatures) in the support region. These histograms include local surface patch (Chen and Bhanu 2007), THRIFT (Flint et al. 2007), point feature histogram (Rusu et al. 2008), fast point feature histogram (Rusu et al. 2009) and signature of histogram of orientations (Tombari et al. 2010b). Apart from the geometric attribute and spatial distribution histogram-based descriptors, Zaharescu et al. (2009) introduced the MeshHoG descriptor, which is analogous to SIFT (Lowe 2004), and uses gradient information to generate a histogram.

The spectral descriptors, such as global point signature (Rustamov 2007), HKS (Sun et al. 2009) and wave kernel signature (WKS) (Aubry et al. 2011), also make up an important category in this area. The descriptors are obtained from the spectral decomposition of the Laplace-Beltrami operator associated with the shape. The Global Point Signature (Rustamov 2007) utilizes the eigenvalues and eigenfunctions of the Laplace-Beltrami operator on the shape to represent the local feature of points. The HKS (Sun et al. 2009) and WKS (Aubry et al. 2011) are based on the heat diffusion process and the temporal evolution of quantum mechanical particles on the shape, respectively.

\subsubsection{Learning-Based 3D Descriptors}

Efforts have also been devoted to generalizing spectral descriptors by using different learning schemes. Litman and Bronstein (2014) generalized the spectral descriptors to a generic family and proposed to learn from examples for obtaining optimized descriptors for a specific task. The learning scheme resembles the spirit of Wiener filter in signal 
processing. Rodolà et al. (2014) proposed a learning method that enables the wave kernel descriptor to recognize a broader class of deformations from the example set by using the random forest classifier. Windheuser et al. (2014) proposed a metric learning method to improve the representation of the spectral descriptors. Modern deep learning techniques have also been successfully applied. Masci et al. (2015) proposed the first attempt and introduced a generalization of the CNN paradigm to non-Euclidean manifolds for shape correspondences. Subsequently, Boscaini et al. proposed to learn descriptors by spectral convolutional networks (Boscaini et al. 2015), and anisotropic CNNs (Boscaini et al. 2016). Monti et al. (2017) proposed a unified framework for generalizing $\mathrm{CNN}$ architectures to non-Euclidean domains (graphs and manifolds). Xie et al. (2016) constructed a deep metric network to form a binary spectral shape descriptor for shape characterization. The input is based on the eigenvalue decomposition of the Laplace-Beltrami operator.

In the spatial domain, the differences of various deep learning methods often lie in the representation of the consumed data. Wei et al. (2016) trained a deep CNN on the depth map representation of shapes to find correspondences. Zeng et al. (2017) proposed to use a 3D deep CNN for learning a local volumetric patch descriptor. This descriptor consumes a voxel grid of truncated distance function values of the local region. Elbaz et al. (2017) proposed a deep neural network auto-encoder to address the 3D matching problem. The authors used a random sphere cover set algorithm to detect feature points and project each local region into a depth map as input to the neural network for producing descriptors. Khoury et al. (2017) parameterized the input by using spherical histograms centered at each point and utilized fully connected networks to generate low-dimensional descriptors. Georgakis et al. (2018) recently employed a Siamese architecture network that processes depth maps. Zhou et al. (2018) proposed to learn from the images of multiple views for the description of 3D keypoints. Wang et al. (2018b) parameterized the multiscale localized neighborhoods of a keypoint into regular 2D grids as the input of a triplet-architecture CNN. Deng et al. (2018) first presented an order-free network on the basis of PointNet (Qi et al. 2017a). This network can consume raw point clouds to exploit the full sparsity in the 3D matching task.

\subsection{Summary}

As previously mentioned, the image patch descriptor is designated to enable accurate and effective correspondence establishment between detected feature points. The objective is to transform the original image information into a discriminative and stable representation that makes the two matched features as close as possible, while the unmatched ones are far apart. To this end, the descriptors should be easy to compute with low computation and storage request. These descriptors should also maintain their discriminative and invariant features against serious deformations and imaging conditions. In the following section, we provide a comprehensive analysis of the handcrafted descriptors and introduce the mechanism by which the learning-based methods can partly address these challenges and achieve promising performance.

Following the construction procedure of traditional local descriptors, the first step is to extract the low-level information, which can be briefly classified into image gradient and intensity. Specifically, the gradient information can be regarded as a higher order image cue than raw intensity. The pooling strategy together with a histogram or statistic manner is often required to form a float descriptor. Thus, this strategy is more invariant to geometrical transformations (perhaps the pool and statistic strategy make it more independent to pixel position and geometrical variety). Nevertheless, it requires additional computation in gradient calculation and statistics as well as the distance measure of float-type data. LBP-based methods typically have high discriminative ability and good robustness to illumination change and image contrast, which are frequently used in texture representation and face recognition.

In contrast with the gradient and/or statistic-based methods, the simple comparison strategy on image intensity would sacrifice great discrimination and robustness. A classical machine learning technique is often designed to identify substantial useful bits. These types of methods are typically in need of the reference orientation estimation to achieve rotation invariant, which appears to be a major error source for most existing methods. However, the use of intensity order is intrinsically invariant to rotation and intensity changes without any geometrical estimation. It can achieve promising performance due to the combination of the use of intensity order and statistical strategy.

Learning-based methods have largely avoided the requirement of manual experience and knowledge priori. They automatically optimize and obtain the optimal parameters and directly construct the wanted descriptor. Traditional learning methods aim to enable the generated descriptors superior in terms of efficiency, low storage, and discrimination. However, the used image cues, such as intensity and gradient, are still with low order, and they highly rely on the framework in handcrafted methods. Nevertheless, the target function, training skills, and datasets that appeared at that time are significant and useful for designing better learningbased methods. Thus, the emergence of deep learning has further advanced this procedure in traditional learning.

Several skills can help improve the discriminability and robustness of deep descriptors. On the one hand, the centralsurround and triplet (even more) structure may provide substantial significant information to learn. The hard negative sample mining strategy would make the structure focused 
on hard samples (may result in overfitting as well) and thus can achieve better matching performance. More reliable loss functions should also be designed according to the basic and intrinsic properties of description task. For instance, recently designed triplet, ranking, contrastive, and global losses, are superior than early simple hinge and cross-entropy losses. On the other hand, valid and comprehensive ground truth datasets are also required for better performance in matching and generalization ability. Training a descriptor together with detectors into the complete matching pipeline through an end-to-end manner has also drawn great attention at present. This can jointly optimize the detector and descriptor, thus can achieve encouraging performance, and the unsupervised training in it can perform without the need of any labeled ground truth patch data. The current descriptors can achieve significant matching performance across image pairs of appearance variances, such as illumination and day-night, by using deep techniques. However, these descriptors still suffer from serious geometrical deformation, such as large rotation or low-overlapped image pair. The low generalization ability for new types of data is also another limitation.

The overall performance of descriptor also depends on the appropriate detector. Different combinations of detectors and descriptors may result in varied matching performance. For this reason, the descriptors should be chosen according to a specific task and the type of image data. The advanced descriptors using deep learning have shown great potential.

\section{Matching Methods}

The matching task aims to establish the correct image pixel or point correspondences between two images with or without using the feature detection and/or description. This task has played a significant role for the entire image matching pipeline. Different definitions of matching task are introduced for specific applications and scenarios and may show their own strengths.

\subsection{Overview of Matching Methods}

Over the past decades in the image matching community, existing methods can be roughly classified into two categories, saying area-based and feature-based (Zitova and Flusser 2003; Litjens et al. 2017). Area-based methods typically refer to dense matching, also known as image registration, which usually do not detect features. In feature-based methods, when the feature points and their local descriptors are extracted from the image pairs, the image matching task could be converted into matching them in indirect and direct ways, which correspond to the use and non-use of the local image descriptors.
Direct feature matching aims to establish the correspondences from two given feature sets by directly using the spatial geometrical relations and optimization methods, which can be roughly classified into graph matching and point set registration. In comparison, indirect feature matching methods typically casts the matching task into a two-stage problem. Such task commonly starts with establishing preliminary correspondences through the similarity of descriptors with the distance judging from the measuring space. Thereafter, the false matches are removed from the putative match sets by using extra local and/or global geometrical constraints. Dense matching from sparse feature correspondences often requires a post-process of transform model estimation, followed by image resampling and interpolation (warping).

We will separate the learning-based methods from areaand feature-based methods and introduce them in a new subsection. From the aspect of input data, learning from images and point data are the two main forms in learning-based matching. These methods can achieve better performance for some scenarios compared to the traditional ones. The matching task in 3-D cases is also briefly introduced in this section.

\subsection{Area-Based Matching}

Area-based methods aim for image registration and establish dense pixel correspondences by directly using the pixel intensity of the entire image. A similarity metric together with an optimization method is in need for geometrical transformation estimation and common area alignment by minimizing the overall dissimilarity between the target and warped moving images. Consequently, several manual similarity metrics are frequently used, including correlation-like, domain transformation, and mutual information (MI) methods. The optimization methods and transform models are also required to perform the final registration task (Zitova and Flusser 2003).

In the image registration community, correlation-like methods, which are regarded as a classical representative in area-based methods, correspond two images by maximizing the similarities of two sliding windows (Zitova and Flusser 2003; Li et al. 2015). For example, the maximum correlation of wavelet features has been developed for automatic registration (Le Moigne et al. 2002). However, this type of method may greatly suffer from the serious image deformations (can only be successfully applied when slight rotation and scaling are presented), windows containing a smooth area without any prominent details, and huge computational burden.

Domain transformed methods tend to align two images on the basis of converting the original images into another domain, such as phase correlation based on Fourier shift theorem (Reddy and Chatterji 1996; Liu et al. 2005; Chen et al. 
1994; Takita et al. 2003; Foroosh et al. 2002), and Walsh transform-based methods (Lazaridis and Petrou 2006; Pan et al. 2008). Such methods are robust against the correlated and frequency-dependent noise and non-uniform, time varying illumination disturbances. Nevertheless, these methods have some limitations in case of image pairs with significantly different spectral contents and small overlap area.

Based on information theory, the MI, such as non-rigid image registration using MI together with B-splines (Klein et al. 2007) and conditional MI (Loeckx et al. 2009), is a measurement of statistical dependency between two images and works with the entire image (Maes et al. 1997). Thus, MI is particularly suitable for the registration of multi-modalities (Chen et al. 2003a, b; Johnson et al. 2001). Recently, Cao et al. (2020) proposed a structure consistency boosting transform to enhance the structural similarity in multi-spectral and multi-modal image registration problem, thus avoiding spectral information distortion. However, the MI exhibits difficulty in determining the global maximum of the entire searching space, inevitably reducing its robustness. Moreover, optimization methods (e.g., continuous optimization, discrete optimization, and their hybrid form) and transformation models (e.g., rigid, affine, thin plate spline (TPS), elastic body, and diffusion models) are considered sufficiently mature. Please refer to Zitova and Flusser (2003), Dawn et al. (2010), Sotiras et al. (2013) and Ferrante and Paragios (2017) for representative literature and further details.

The area-based methods are acceptable for medical or remote sensing image registration, which many feature-based methods are not workable anymore because the images often contain less textural details and large variance of image appearance due to the different imaging sensors. However, the area-based methods may greatly suffer from the serious geometrical transformations and local deformations. While deep learning has proven its efficacy, in which the early ones are usually employed as a direct extension of the classical registration framework, and later ones use a reinforcement learning paradigm to iteratively estimate the transformation, even directly estimate the deformative field in an end-to-end manner. The area-based matching with learning strategies will be reviewed in the part of learning-based matching.

\subsection{Graph Matching Methods}

Given the feature points extracted from an image, we can construct a graph by associating each feature point to a node and specifying edges. This procedure naturally provides convenience to investigate the intrinsic structure of image data, especially for the matching problem. By this definition, graph matching (GM) refers to the establishment of node-to-node correspondences between two or multiple graphs. For its importance and fundamental challenge, GM has been a long-standing research area over decades and is still of great interest to researchers. From the problem setting perspective, GM can be divided into two categories, namely, exact and inexact matching. Exact matching methods consider GM to be a special case of the graph or subgraph isomorphism problem. It aims to find the bijection of two binary (sub)graphs; consequently, all edges are strictly preserved babai2018groups, cook2006mining,levi1973note). In fact, this requirement is too strict for real-world tasks like computer vision. Hence researchers often resort to inexact matching with weighted attributes on nodes and edges. Such an approach enjoys good flexibility and utility in practice. Therefore, we primarily concentrate on the review of inexact matching methods in this survey.

To some extent, GM possesses a simple yet general formulation of the feature matching problem, which encodes the geometrical cues into the node affinities (first-order relations) and edge affinities (second-order relations) to deduce the true correspondences between two graphs. Aside from the geometrical cues, the high-level information of feature points can also be incorporated in GM (e.g. descriptor similarities as node affinities). This information only serves as a supplementary one and is not necessarily required. In the general and recent form, GM can be formulated as a Quadratic Assignment Problem (QAP) (Loiola et al. 2007). Although different forms exist in the literature, the main body of research has focused on the Lawler's QAP (Lawler 1963). Given two graphs $G_{1}=\left(V_{1}, E_{1}\right)$ and $G_{2}=\left(V_{2}, E_{2}\right)$, where $\left|V_{1}\right|=n_{1}$, $\left|V_{2}\right|=n_{2}$, each node $v_{i} \in V_{1}$ or $v_{j} \in V_{2}$ represents a feature point, and each edge $e_{i} \in E_{1}$ or $e_{j} \in E_{2}$ is defined over a pair of nodes. Without loss of generality we assume $n_{1} \geq n_{2}$, Lawler's QAP formulation of GM then can be written as:

$$
\begin{aligned}
& \max J(\mathbf{X})=\operatorname{vec}(\mathbf{X})^{\top} \mathbf{K} \operatorname{vec}(\mathbf{X}), \\
& \text { s.t. } \mathbf{X} \in\{0,1\}^{n_{1} \times n_{2}}, \mathbf{X} \mathbf{1}_{n_{2}} \leq \mathbf{1}_{n_{1}}, \mathbf{X}^{\top} \mathbf{1}_{n_{1}}=\mathbf{1}_{n_{2}},
\end{aligned}
$$

where $\mathbf{X}$ denotes the permutation matrix, i.e. $\mathbf{X}_{i j}=1$ indicates that node $v_{i} \in V_{1}$ corresponds to node $v_{j} \in V_{2}$ and $\mathbf{X}_{i j}=0$ otherwise, $\operatorname{vec}(\mathbf{X})$ denotes the column-wise vectorization of $\mathbf{X}$, and $\mathbf{1}_{n_{1}}$ and $\mathbf{1}_{n_{2}}$ respectively denote the column vectors of all ones, $\mathbf{K}$ denotes the affinity matrix, whose diagonal and non-diagonal entries encode the first-order and second-order edge affinities between the two graphs. No universal approach can be utilized to construct the affinity matrix; however, a simple strategy is to use the similarities of feature descriptors [e.g. Shape Context (Belongie et al. 2001)] and differences of edge length to determine node and edge affinities.

The Koopmans-Beckmann's QAP is another popular formulation. The form is different from Lawler's QAP as expressed as:

$$
J(\mathbf{X})=\operatorname{tr}\left(\mathbf{K}_{p}^{\top} \mathbf{X}\right)+\operatorname{tr}\left(\mathbf{A}_{1} \mathbf{X} \mathbf{A}_{2} \mathbf{X}^{\top}\right)
$$


where $\mathbf{A}_{1}$ and $\mathbf{A}_{2}$ are the weighted adjacency matrices of the two graphs, respectively, and $\mathbf{K}_{p}$ is the node affinity matrix. In Zhou and De la Torre (2015), the relation between Koopmans-Beckmann's and Lawler's QAP has been investigated, which reveals that Koopmans-Beckmann's QAP can be regarded as a special case of Lawler's.

The GM problem is translated into finding the optimal one-to-one correspondences $\mathbf{X}$ that maximizes the overall affinity score $J(\mathbf{X})$. As a combinatorial QAP problem in general, GM is known to be NP-hard. Most methods relax the stringent constraints and provide approximate solutions in an affordable over head. In this regard, many relaxation strategies are introduced in the literature, thereby leading to a variety of GM solvers. In the following, we briefly review the influential ones through the development course of GM.

\subsubsection{Spectral Relaxations}

The first group of methods follow a strategy of spectral relaxation. Leordeanu and Hebert (2005) proposed to replace the one-to-one mapping constraint and the binary constraint by constraining $\|\operatorname{vec}(\mathbf{X})\|_{2}^{2}=1$. In this case, the solution $\mathbf{X}$ can be obtained by solving an eigenvector problem. Each element in $\mathbf{X}$ is interpreted as the association of one correspondence with the optimal cluster (true correspondences). A discretization strategy is used to enforce the mapping constraints. The idea was later improved by Cour et al. (2007), who explicitly considered enforcing the one-to-one mapping constraint to achieve tighter relaxation. This method can also be solved in closed forms as an eigenvector problem. Liu and Yan (2010) proposed to detect multiple visual patterns by using a $l_{1}$-norm-based spectral relaxation technique, i.e. constraining $\|\operatorname{vec}(\mathbf{X})\|_{1}=1$. The solution can be efficiently obtained by replicator equation from evolutionary game theory. Jiang et al. (2014) presented a non-negative matrix factorization technique, which extends the constraint as $\|\operatorname{vec}(\mathbf{X})\|_{p}=1, p \in[1,2]$. Meanwhile, Egozi et al. (2012) presented a fairly different approach. In their work, they provided a probabilistic interpretation of spectral matching schemes and derived a novel probabilistic matching scheme wherein the affinity matrix is also updated in the iteration process. With Koopmans-Beckmann's QAP formulation, the spectral methods (Umeyama 1988; Scott and Longuet-Higgins 1991; Shapiro and Brady 1992; Caelli and Kosinov 2004) relax $\mathbf{X}$ to be orthogonal, i.e. $\mathbf{X}^{\top} \mathbf{X}=\mathbf{I}$. This expression can be solved in a closed form as an eigenvalue problem. These methods possess the merit of efficiency due to the loose relaxation. However, the accuracy is not advantaged in general.

\subsubsection{Convex Relaxations}

Many studies have turned to investigating convex relaxations of the original problem to obtain theoretical advantages for solving the non-convex QAP issue. Strong convex relaxations can be obtained by lifting methods that add auxiliary variables representing quadratic monomials in the original variables. This enables the addition of additional convex constraints on the lifted variables. Semi-definite programming (SDP) is a general tool for combinatorial problems and has been applied to solving GM (Schellewald and Schnörr 2005; Torr 2003; Zhao et al. 1998; Kezurer et al. 2015). The SDP relaxation is quite tight and allows finding a strong approximation in polynomial time. However, the high computational cost prohibits its scalability. Some other lifting methods with linear programming relaxations have also been developed (Almohamad and Duffuaa 1993; Adams and Johnson 1994). The dual problem of the LP relaxations are recently extensively considered to solve GM (Swoboda et al. 2017; Chen and Koltun 2015; Swoboda et al. 2017; Torresani et al. 2012; Zhang et al. 2016), which has a strong link with the MAP inference algorithms.

\subsubsection{Convex-to-Concave Relaxations}

One useful strategy is to utilize the path-following technique. This approach gradually achieves a convex-to-concave procedure of the original problem to finally find a good solution with the constraints satisfied. The computational complexity is also much lower than those of the lifting methods. Zaslavskiy et al. (2009) adopted this strategy for GM problem with Koopmans-Beckmann's QAP formulation, which is extended by to directed graphs (Liu et al. 2012b) and partial matching (Liu and Qiao 2014). Zhou and De la Torre (2015) presented a unified framework of GM based on the factorization of affinity matrix based on Lawler's QAP. Such a framework effectively reduces the computational complexity and reveals the relation between Koopmans-Beckmann's and Lawler's QAPs. The (advanced) doubly stochastic (DS) relaxation methods improve upon these approaches by identifying tighter formulations (Fogel et al. 2013; Dym et al. 2017; Bernard et al. 2018), where the tightness of spectral, SDP, and DS relaxation is discussed and theoretically verified. 


\subsubsection{Continuous Relaxations}

A large volume of GM methods has focused on devising accurate or efficient algorithms to solve the QAP approximately, albeit with no global optimality guarantee. In most cases, $\mathbf{X}$ is simply relaxed to be continuous, as a DS matrix. Gold and Rangarajan (1996) proposed a graduated assignment algorithm, which performs gradient ascent on the relaxed problem under an annealing schedule. The convergence of this method has been revisited and improved by Tian et al. (2012) with a soft constrained mechanism. van Wyk and van Wyk (2004) proposed to enforce the one-to-one mapping constraint by successively projecting onto the convex set of the desired integer constraints. Leordeanu et al. (2009) proposed an efficient algorithm that optimizes in the (quasi) discrete domain via solving a sequence of linear assignment problems. Many famous optimization techniques, such as ADMM (Lê-Huu and Paragios 2017), tabu search (Adamczewski et al. 2015) and multiplicative update algorithm (Jiang et al. 2017a), have also been tested. Recent studies also include Jiang et al. (2017b) and Yu et al. (2018), which introduce new schemes to asymptotically approximate the original QAP, and Maron and Lipman (2018), which presents a new (probably) concave relaxation technique. Yu et al. (2020b) introduced a determinant regularization technique together with gradient-based optimization to relax this problem into continuous domain.

\subsubsection{Multi-graph Matching}

In contrast to the classic two-graph matching setting, jointly matching a batch of graphs with consistent correspondences, i.e. multi-graph matching, has recently drawn increasing attention due to its methodological advantage and potential to incorporate cross-graph information. Arguably, one central issue of multi-graph matching lies in the enforcement of cycle-consistency for a feasible solution. In general, this concept refers to the fact that the bijection correspondence between two graphs shall be consistent with a derived one through an intermediate graph. Put it more concretely, for any pair of graphs $G_{a}$ and $G_{b}$ with their node correspondence matrix $\mathbf{X}^{a b}$, let $G_{c}$ be an intermediate graph, the cycle consistency constraint is enforced: $\mathbf{X}^{a c} \mathbf{X}^{c b}=\mathbf{X}^{a b}$, where $\mathbf{X}^{a c}$ and $\mathbf{X}^{c b}$ are the matching solutions of $G_{a}$ and $G_{c}$ and $G_{c}$ and $G_{b}$, respectively.

Existing multi-graph matching methods can be roughly grouped into three lines of works. For the methods falling into the first group, the multi-graph matching problem is solved by an iterative procedure for computing a number of two-graph matching tasks (Yan et al. 2013, 2014, 2015a, b; Jiang et al. 2020b). In each iteration, a two-graph matching solution is computed to locally maximize the affinity score, which can leverage off-the-shelf pairwise matching solvers, such as in Jiang et al. (2020b), both offline batch mode and online setting are considered to explore the concept of cycleconsistency over pairwise matching. Another body of work takes the initial (noisy) pairwise matching result as input, and aims to recover a globally consistent pairwise matching set (Kim et al. 2012; Pachauri et al. 2013; Huang and Guibas 2013; Chen et al. 2014; Zhou et al. 2015; Wang et al. 2018; $\mathrm{Hu}$ et al. 2018). In these methods, matching over all graphs is jointly and equally considered to form a bulk matrix that includes all pairwise matchings. The intrinsic structure of this matrix induced by the matching problem, such as cycleconsistency, is investigated. The last group utilizes clustering or low rank recovery techniques to solve multi-graph matching, which provides a new perspective in the feature space for the problem (Zeng et al. 2012; Yan et al. 2015c, 2016a; Tron et al. 2017). More recently, the multi-graph matching problem has been considered in the optimization framework with a theoretically well-grounded convex relaxation (Swoboda et al. 2019), or with projected power iterations to search for a feasible solution (Bernard et al. 2019).

\subsubsection{Other Paradigms}

Although the QAP formulation is prevalent in GM, the way of formulation is not unique. Numerous methods deal with GM from different perspectives or paradigms and also form an important category in this field.

Cho et al. (2010) provided a random walk view of GM and devised a technique to obtain solution by simulating random walks on the association graph. Lee et al. (2010) and Suh et al. (2012) introduced Monte Carlo methods to improve the matching robustness. Cho and Lee (2012) further devised a progressive GM method, which combines progression of graphs with matching of graphs to reduce the computational complexity. Wang et al. (2018a) proposed to use a functional representation of graphs and conduct matching by minimizing the discrepancy between the original and the transformed graphs. Subsequently, in order to suppress the matching of outliers, Wang et al. (2020) assigned zerovalued vectors to the potential outliers in the obtained optimal correspondence matrix. The affinity matrix plays a key role in the GM problem. However, the handcrafted $\mathbf{K}$ is vulnerable to scale and rotation differences. To this end, unsupervised (Leordeanu et al. 2012) and supervised (Caetano et al. 2009) methods are devised to learn $\mathbf{K}$. Zanfir and Sminchisescu (2018) recently addressed this issue with an end-to-end deep learning scheme. Wang et al. (2020) introduced a fully trainable framework for graph matching. In this framework, they utilized a graph network block module and simultaneously considered the learning of node/edge affinities and the solving of combinatorial optimization. 
The extension of GM to a high-order formulation is a natural way to improve the robustness by mostly exploring the geometrical cues. This leads to a tensor-based objective (Lee et al. 2011) also called hypergraph matching:

$J_{H}(\mathbf{X})=\mathbf{H} \otimes_{1} \mathbf{x} \otimes_{2} \mathbf{x} \ldots \otimes_{m} \mathbf{x}$

where $m$ is the order of affinities, $\mathrm{H}$ denotes the $m$-order tensor encoding the affinities between hyperedges in the graphs, $\otimes_{k}$ is the tensor product, and $\mathbf{x}=\operatorname{vec}(\mathbf{X})$. Representative studies on hypergraph matching include Zass and Shashua (2008), Chertok and Keller (2010), Lee et al. (2011), Chang and Kimia (2011), Duchenne et al. (2011) and Yan et al. (2015d).

\subsection{Point Set Registration Methods}

Point set registration (PSR) aims to estimate the spatial transformation that optimally aligns two point sets. In feature matching, different formulations are adopted in PSR and GM. For two point sets, GM methods determine the alignment via maximizing the overall affinity score of unary correspondence and pairwise correspondences. By contrast, PSR methods determine the underlying global transformation. Given the two point sets $\left\{\mathbf{x}_{i}\right\}_{i=1}^{n_{1}}$ and $\left\{\mathbf{y}_{i}\right\}_{i=1}^{n_{2}}$, the general conventional objective can be expressed as

$$
\begin{aligned}
& \min J(\mathbf{P}, \boldsymbol{\theta})=\sum_{i, j} p_{i j}\left\|\mathbf{y}_{j}-T\left(\mathbf{x}_{i}, \boldsymbol{\theta}\right)\right\|_{2}^{2}+g(\mathbf{P}) \\
& \text { s.t. } \boldsymbol{\theta} \in \Theta, \mathbf{P} \in\{0,1\}^{n_{1} \times n_{2}}, \mathbf{P} \mathbf{1}_{n_{2}} \leq \mathbf{1}_{n_{1}}, \mathbf{P}^{\top} \mathbf{1}_{n_{1}} \leq \mathbf{1}_{n_{2}},
\end{aligned}
$$

where $\boldsymbol{\theta}$ denotes the parameters of the predefined transformation. The regularization term $g(\mathbf{P})$ avoids trivial solutions, such as $\mathbf{P}=\mathbf{0}$. Compared to GM, this model only represents the general principles, but does not necessarily cover all the algorithms for PSR. For example, a probabilistic interpretation or a density-based objective can be used, and the constraints for $\mathbf{P}$ may be only partially imposed during optimization, which all differ from the above formulation.

PSR poses a stronger assumption on the data, that is, the existence of a global transformation between point sets, which is the key feature that differentiates it from GM. Although the generality is restricted, this assumption leads to low computational complexity because of the few parameters needed for global transformation models. A sophisticated transformation model is developed from rigid to non-rigid ones in order to enhance the generalization ability. Various schemes are also proposed to improve robustness against degradations, such as noise, outliers, and missing points.

\subsubsection{ICP and Its Variants}

PSR has been an important research topic for the last few decades in computer vision, and the iterative closest point
(ICP) algorithm is a popular method (Besl and McKay 1992). ICP iteratively alternates between hard assignments of correspondences for the closest points in two point sets and the closed-form rigid transformation estimation until convergence. The ICP algorithm is widely used as baselines due to its simplicity and low computational complexity. However, a good initialization is required because ICP is prone to be trapped into local optima. Numerous studies, such as EM-ICP (Granger and Pennec 2002), LM-ICP (Fitzgibbon 2003), and TriICP (Chetverikov et al. 2005), in the research field of PSR have been proposed to improve ICP. The reader is referred to a recent survey (Pomerleau et al. 2013) for a detailed discussion of ICP's variants. The robust point matching (RPM) algorithm (Gold et al. 1998) are proposed to overcome the ICP limitations; the soft assignment and deterministic annealing strategy are adopted, and the rigid transformation model is generalized to a non-rigid one by using the thin-plate spline [TPS-RPM (Chui and Rangarajan 2003)].

\subsubsection{EM-Based Methods}

RPM is also a representative of the EM-like PSR methods, which form an important category in this field. The EM-like methods formulate PSR as an optimization problem of either a weighted squared loss function or the log-likelihood maximization of Gaussian mixture models (GMMs), and local optimum is searched through EM or EM-like algorithms. The posterior probability of each correspondence is computed in the E-step, and the transformation is refined in the M-step. Sofka et al. (2007) investigated the modeling of uncertainty in the registration process and presented a covariance driven correspondence method in an EM-like framework. Myronenko and Song (2010) proposed the well-known coherent point drift (CPD) method in which a probabilistic framework is established on the basis of GMM; here, the EM algorithm is utilized for maximum likelihood estimation of the parameters. Horaud et al. (2011) developed an expectation conditional maximization-based probabilistic method, which allows the use of anisotropic covariance for the mixture model components and improves over isotropic covariance case. Ma et al. (2016b) and Zhang et al. (2017a) exploited the unification of local feature and global feature in the GMMbased probabilistic framework. Lawin et al. (2018) presented a density adaptive PSR method via modeling the underlying structure of the scene as a latent probability distribution.

\subsubsection{Density-Based Methods}

Density-based methods introduce generative models to the PSR problem, in which no explicit point correspondence is established. Each point set is represented by a density function, such as GMM. Registration is achieved by the mini- 
mization of a statistical discrepancy measure between the two density functions. Tsin and Kanade (2004) were the first to propose such a method and used kernel density functions to model the point sets, and the discrepancy measure is defined as kernel correlation. Meanwhile, Glaunes et al. (2004) represented the point sets by using relaxed Dirac delta functions. They then determined the optimal diffeomorphic transformation that minimizes the distance of the two distributions. Jian and Vemuri (2011) extended this approach by using GMMbased representation and minimizing the $\mathrm{L} 2$ error between the densities. The authors also provided a unified framework of density-based PSR. Many popular methods, including Myronenko and Song (2010) and Tsin and Kanade (2004) can be regarded as special cases in theory. Campbell and Petersson (2015) proposed to use a support vector parameterized GMM for adaptive data representation. This approach can improve the robustness of density-based methods to noise, outliers, and occlusions. Recently, Liao et al. (2020) utilized fuzzy clusters to represent a scanned point set, then registed two point sets by minimizing a fuzzy weighted sum of distances between their fuzzy cluster centers.

\subsubsection{Optimization-Based Methods}

A group of optimization-based methods have been proposed as globally optimal solutions to alleviate the local optimum issue. These methods generally search in a limited transformation space for timing saving, such as rotation, translation, and scaling. Stochastic optimization techniques, including genetic algorithms (Silva et al. 2005; Robertson and Fisher 2002), particle swarm optimization ( $\mathrm{Li}$ et al. 2009), particle filtering (Sandhu et al. 2010) and simulated annealing schemes (Papazov and Burschka 2011; Blais and Levine 1995), are widely used, but no convergence is guaranteed. Meanwhile, Branch and bound $(\mathrm{BnB})$ is a well-established optimization technique that can efficiently search the globally optimal solution in the transformation space and form the theoretical basis of many optimization-based methods, including Li and Hartley (2007), Parra Bustos et al. (2014), Campbell and Petersson (2016), Yang et al. (2016) and Liu et al. (2018b). In addition to these methods, Maron et al. (2016) introduced a semidefinite programming (SDP) relaxation-based method, in which a global solution is guaranteed for isometric shape matching. Lian et al. (2017) formulated PSR as a concave QAP by eliminating the rigid transformation variables, and $\mathrm{BnB}$ is utilized to achieve a globally optimal solution. Yao et al. (2020) presented a formulation for robust non-rigid PSR based on a globally smooth robust estimator for data fitting and regularization, which is optimized by majorization-minimization algorithm to reduce each iteration in solving a simple least-squares problem. Another method in Iglesias et al. (2020) presents a study of global optimality conditions for PSR with missing data. This method applies Lagrangian duality to generate a candidate solution for the primal problem thus enables it to obtain the corresponding dual variable in a closed form.

\subsubsection{Miscellaneous Methods}

Apart from the commonly used rigid model or non-rigid transformation model based on TPS (Chui and Rangarajan 2003) or Gaussian radial basis functions (Myronenko and Song 2010), additional complex deformations are also considered in the literature. These models include simple articulated extensions, such as Horaud et al. (2011) and Gao and Tedrake (2019). A smooth locally affine model is introduced as the transformation model and developed under the ICP framework in non-rigid ICP (Amberg et al. 2007), which is also adopted in Li et al. (2008). However, this model should be used in conjunction with sparse hand selected feature correspondences as it allows many degrees of freedom. A different linear skinning model, which does not require user's involvement in the registration process, has been proposed and applied in another work (Chang and Zwicker 2009).

Another line of PSR methods introduce shape descriptors into the registration process. Local shape descriptors, such as spin images (Johnson and Hebert 1999), shape contexts (Belongie et al. 2001), integral volume (Gelfand et al. 2005) and point feature histograms (Rusu et al. 2009) are generated. Sparse feature correspondences are established by a similarity constraint of descriptors. Subsequently, the underlying rigid transformation can be estimated using random sampling consensus (RANSAC) (Fischler and Bolles 1981) or BnB search (Bazin et al. 2012). Ma et al. (2013b) proposed a robust algorithm based on the $L_{2} E$ estimator in a non-rigid case.

Some new schemes for PSR based on different observations have emerged. Golyanik et al. (2016) modeled point set as particles with gravity as attractive force, and registration is accomplished by solving the differential equations of Newtonian mechanics. Ma et al. (2015a) and Wang et al. (2016) proposed the use of context-aware Gaussian fields to address the PSR problem. Vongkulbhisal et al. $(2017,2018)$ proposed the discriminative optimization method. This approach learns the search direction from training data to guide optimization without the need of defining cost functions. Danelljan et al. (2016) and Park et al. (2017) considered the color information of point sets, whereas Evangelidis and Horaud (2018) and Giraldo et al. (2017) addressed the problem of joint registration of multiple point sets.

\subsection{Descriptor Matching with Mismatch Removal}

Descriptor matching followed by mismatch removal, also called indirect image matching, casts the matching task into a two-stage problem. This method commonly starts with estab- 
lishing preliminary correspondences through the similarity of local image descriptors with the distance judging from the measuring space. Several common strategies, including fixed threshold (FT), nearest neighbor (NN) also called brute force matching, mutual NN (MNN), and NN distance ratio (NNDR), are available for the construction of putative match sets. Thereafter, the false matches are removed from the putative match sets by using extra local and/or global geometrical constraints. We briefly divide the mismatch removal methods into resampling-based, non-parametric model-based, and relaxed methods. In the following sections, we will introduce these methods in detail and provide comprehensive analysis.

\subsubsection{Putative Match Set Construction}

Suppose that we have detected and extracted $M$ and $N$ local features to be matched from the considering two images $I_{1}$ and $I_{2}$. The descriptor matching stage operates by computing the pairwise distance matrix with $M \times N$ entries and then selecting the potential true matches through the aforementioned rule.

The FT strategy considers the matches with their distances below a fixed threshold. However, this strategy can be sensitive and may incur numerous one-to-many matchings in contrast to the one-to-one correspondence nature. This situation results in poor performance in feature matching task. The NN strategy can effectively deal with the data sensitivity problem and recall more potential true matches. Such a strategy has been applied in various descriptor matching methods, but it cannot avoid the one-to-many cases. In mutual NN descriptor matching, each feature in $I_{1}$, looks for its $\mathrm{NN}$ in $I_{2}$ (and vice versa), and the feature pairs that are mutual $\mathrm{NN}$ become candidate matches in the putative match set. This type of strategy can obtain high ratio of correct matches but may sacrifice many other true correspondences. The NNDR considered that the distance difference between first and second NN is significant. Hence, the use of the distance ratio with a predefined threshold would obtain robust and promising matching performance while not sacrifice many true matches. However, NNDR relies on the stable distance distribution of these descriptors even though the method is widely used and well performed in SIFT-like descriptor matching. In fact, NNDR is no longer applicable for descriptors of other types, such as binary or some learning based descriptors (Rublee et al. 2011; Ono et al. 2018).

The optimal choice of these methods for descriptor matching should rely on the property of descriptor and the specific application. For example, the MNN is stricter than others with high inlier ratio but may sacrifice many other potential true matches. By contrast, NN and NNDR tend to be more general in feature matching task with relatively better performance. Mikolajczyk and Schmid (2005) proposed a simple test about these candidate match selection strategies. Although various approaches are available for putative feature correspondence construction the use of only local appearance information and simple similarity-based putative match selection strategies, will unavoidably result in a large number of incorrect matches, particularly when images undergo serious non-rigid deformation, extreme viewpoint changes, low quality, and/or repeated contents. Therefore, a robust, accurate, and efficient mismatch elimination method is urgently required in the second stage to preserve as many true matches as possible while keeping the mismatch to a minimum by using additional geometrical constraints.

\subsubsection{Resampling-Based Methods}

Resampling technique is (arguably) a prevalent paradigm and is represented by the classic RANSAC algorithm (Fischler and Bolles 1981). Basically, the two images are assumed to be coupled by a certain parametric geometric relation, such as projective transformation or epipolar geometry. The RANSAC algorithm then follows a hypothesize-and-verify strategy: repeatedly sample a minimal subset from the data, e.g. four correspondences for projective transformation and seven correspondences for fundamental, estimate a model as hypothesis, and verify the quality by the number of consistent inliers. Finally, the correspondences consistent with the optimal model are recognized as inliers.

Various methods have been proposed to improve the performance of RANSAC. In MLESAC (Torr and Zisserman 1998, 2000), the model quality is verified by a maximum likelihood process, which albeit under certain assumptions, can improve the results and is less sensitive to the predefined threshold. The idea of modifying the verification stage is not only utilized but also further extended in many following studies due to the simple implementation. The modification of sampling strategy has also been considered in quite a few studies due to the appealing result of efficiency enhancement. In essence, diverse prior information is incorporated to increase the probability of selecting an all-inlier sample subset. Specifically, the inliers are assumed to be spatially coherent in NAPSAC (Nasuto and Craddock 2002), or exist with some groupings in GroupSAC (Ni et al. 2009). PROSAC (Chum and Matas 2005) exploits a priori predicted inlier probability, and EVSAC (Fragoso et al. 2013) uses an estimate of confidence with extreme value theory of the correspondences. Another seminal work is the locally optimized RANSAC (LO-RANSAC) (Chum et al. 2003), with the key observation that taking minimal subsets can amplify the underlying noise and yield hypotheses that are far from the ground truth. This problem is addressed by introducing a local optimization procedure when arriving at the so-farthe-best model. In the original paper, local optimization is implemented as an iterated least squares fitting process with a shrinking inlier-outlier threshold inside an inner RANSAC. 
This has a large-than-minimal sampling and is applied only to the inliers of the current model. The computational cost issue of LO-RANSAC is addressed in Lebeda et al. (2012), where several implementation improvements are suggested. The local optimization step is augmented with a graph-cut technique in Barath and Matas (2018). Many improving strategies for RANSAC are integrated in USAC (Raguram et al. 2012).

More recently, Barath et al. (2019b) applyed $\sigma$-consensus in their MAGSAC, to eliminate the need of a user-defined threshold by marginalizing over a range of noise scales. Whereafter, observing that nearby points are more likely to originate from the same geometric model, Barath et al. (2019a) extracted the local structure for global sampling and parameter model estimation by drawing samples from gradually growing neighborhoods. Based on above two methods, they introduced MAGSAC++ (Barath et al. 2020) with a new scoring function. This method avoids requiring the inlieroutlier decision, in which a novel marginalization procedure formulated as an M-estimation is solved by an iteratively re-weighted least squares procedure, and the progressive growing sampling strategy in Barath et al. (2019a) is also applied for RANSAC-like robust estimation.

Some fundamental shortcomings are exhibited by the resampling methods despite their efficacy in wide applications of computer vision. For example, the theoretically required runtime exponentially grows with the increase of outlier rate. The minimal subset sampling strategy only applies to parametric models and fails to handle image pairs undergoing complex transformations, such as non-rigid ones. This situation motivates researchers to develop new algorithms divorced from the resampling paradigm.

\subsubsection{Non-parametric Model-Based Methods}

A group of non-parametric model-based methods have been proposed. Instead of simple parametric models, nonparametric models address more general priors in matching, e.g. motion coherence, and can deal with degenerated scenarios. These methods are distinguished by different deformation functions to model the transformation and different means to cope with gross outliers. Pilet et al. (2008) proposed the use triangulated 2-D mesh to model the deformation using a tailored robust estimator for eliminating the detrimental effect of outliers. The idea of robust estimators is also leveraged in Gay-Bellile et al. (2008), with Huber estimator, and Ma et al. (2015), with $L_{2} E$ estimator, despite of their different modeling of deformation. A fairly different method is proposed in Li and Hu (2010), in which the Support Vector Regression technique is employed to robustly estimate a correspondence function and reject mismatches.

The seminal work vector field consensus (VFC) (Ma et al. 2013a, 2014) introduces a new framework for nonrigid matching. The deformation function is restricted within the reproducing kernel Hilbert space in association with Tikhonov regularization to enforce the smoothness constraint. The estimation is conducted in a Bayesian model, where the outliers are explicitly considered for robustness. The VFC algorithm, and its variants (Ma et al. 2015b, 2017a, 2019b) have been proven effective.

\subsubsection{Relaxed Methods}

The recent trend has been towards developing relaxed methods for matching, where the geometric constraint is made less strict to accommodate even complex scenarios, such as motion discontinuities arising from image pairs of wide baselines or with objects undergoing independent motions. Certain GM methods (Leordeanu and Hebert 2005; Liu and Yan 2010) are available for such requirements and use quadratic models that incorporate pairwise geometric relations of correspondences to find the potentially correct ones. However, the results are often coarse.

Lipman et al. (2014) considered deformations that are piecewise affine; they then formulated feature matching into a constrained optimization problem that seeks for such a deformation consistent with the most correspondences and exerts a bounded distortion. Lin et al. $(2014,2017)$ proposed to identify true matches with likelihood functions estimated using nonlinear regression technique in a specially designed domain of correspondence, where motion coherence is imposed, while discontinuities are also allowed. This concept corresponds to enforcing a local motion coherence constraint. Ma et al. (2018a, 2019d) presented a locality preserving approach for matching, whereby a global distortion model for matching is relaxed to focus on the locality of each correspondence in exchange for generality and efficiency. The derived criterion has been proven able to rapidly and accurately filter erroneous matches. A similar method appeared in Bian et al. (2017) wherein a simple criterion based on local supporting matches to reject outliers is introduced. Jiang et al. (2020a) casted feature matching as a spatial clustering problem with outliers to adaptively cluster the putative matches into several motion consistent clusters together with an outlier/mismatch cluster. Another method in Lee et al. (2020) formulates the feature matching problem as a Markov random field that uses both local descriptor distance and relative geometric similarities to enhance the robustness and accuracy.

\subsection{Learning for Matching}

Apart from detectors or descriptors, learning-based matching methods are commonly used to substitute traditional methods in information extraction and representation or model regression. The matching step by learning can be roughly classified into image-based and point-based learning. Based 
on the traditional methods, the former aims to cope with three typical tasks, namely image registration (Wu et al. 2015a), stereo matching (Poursaeed et al. 2018) and camera localization or transformation estimation (Poursaeed et al. 2018; Erlik Nowruzi et al. 2017; Yin and Shi 2018). Such a method can directly realize task-based learning without attempting to detect any salient image structure (e.g. interest points) in advance. By contrast, point-based learning prefers conducting on the extracted point sets; such methods are commonly used for point data processing, such as classification, segmentation (Qi et al. 2017a,b) and registration (Simonovsky et al. 2016; Liao et al. 2017). Researchers have also used these for correct match selection and geometrical transformation model estimation from putative match sets (Moo Yi et al. 2018; Ma et al. 2019a; Zhao et al. 2019; Ranftl and Koltun 2018; Poursaeed et al. 2018).

\subsubsection{Learning from Images}

Matching methods of image-based learning often use CNNs for image-level latent information extraction and similarity measurement, as well as geometrical relation estimation. Therefore, the patch-based learning (Sect. 3.3: learningbased feature descriptors) is frequently used as an extension of area-based image registration and stereo matching. This is because traditional similarity measurements in a sliding window can be easily replaced with a deep manner, i.e., deep descriptors. However, the success achieved by researchers in using deep learning in spatial transformation networks (STN) (Jaderberg et al. 2015) and optical flow estimation (FlowNet) (Dosovitskiy et al. 2015) has aroused a wave of studies on directly estimating the geometrical transformation or nonparametric deformation field with deep learning techniques, even achieving an end-to-end trainable framework.

Image registration. For area-based image registration, early deep learning is generally used as a direct extension of the classical registration framework, and later use the reinforcement learning paradigm to iteratively estimate the transformation, even directly estimate the deformative field or displacement field for the registration task. The most intuitional approach is to use deep learning networks to estimate the similarity measurement for the target image pair in order to drive an iterative optimization procedure. In this way, the classical measure metrics, such as the correlation-like and MI methods, etc., can be substituted with more superior deep metrics. For instance, Wu et al. (2015a) achieved deformable image registration by using the convolutional stacked auto-encoder (CAE) to discover compact and highly discriminative features from the observed image patch data for similarity metrics learning. Similarly, to obtain better similarity measure, Simonovsky et al. (2016) used a deep network trained from a few aligned image pairs. In addition, a fast, deformable image registration method called
Quicksilver (Yang et al. 2017b) has been devised by the patch-wise prediction of a deformation model directly using image appearance, whereby a deep encoder-decoder network is used for predicting the large deformation diffeomorphic model. Inspired by deep convolution, Revaud et al. (2016) introduced a dense matching algorithm based on a hierarchical correlation architecture. This method can handle complex non-rigid deformations and repetitive textured regions. Arar et al. (2020) introduced an unsupervised multi-modal image registration technique based on an image-to-image translation network with geometric preserving constraints.

Different from metric learning, a trained agent is used for image registration with a reinforcement learning paradigm, and typically for estimating a rigid transformation model or a deformable field. Liao et al. (2017) first used the reinforcement learning for rigid image registration, in which an artificial agent and a greedy supervised approach coupled with attention-driven hierarchical strategy are used to realize the "strategy learning" process and find the best sequence of motion actions to yield image alignment. An artificial agent, which explores the parametric space of a statistical deformation model by training from a large number of synthetically deformed image pairs, is also trained in Krebs et al. (2017) to cope with deformable registration problem and the difficulty in extracting reliable ground-truth deformable fields of real data. Instead of using a single agent, Miao et al. (2018) proposed a multi-agent reinforcement learning paradigm for medical image registration in which the auto-attention mechanism is used for receptive multiple image regions. However, the reinforcement learning is often used to predict iterative updates of the regression procedure and still consumes large computation in the iterative process.

To reduce the run time and avoid explicitly defining a dissimilarity metric, end-to-end registration in one shot has received increasing attention. Sokooti et al. (2017) first designed deep regression networks to directly learn a displacement vector field from a pair of input images. Another method in de Vos et al. (2017) similarly trained a deep network to regress and output the parameters of spatial transformation, which can then generate the displacement field to warp the moving image to the target image. However, a similarity metric between image pairs is still required to achieve unsupervised optimization. More recently, a deep learning framework has been introduced in de Vos et al. (2019) for unsupervised affine and deformable image registration. The trained networks can be used to register pairs of unseen images in one shot. Similar methods regarding deep networks as a regressor can directly learn the parameter transform model from image pairs, such as Fundamental (Poursaeed et al. 2018), Homography (DeTone et al. 2016), and non-rigid deformation (Rocco et al. 2017).

Many other end-to-end image level learning-based registration methods are presented. Chen et al. (2019) pro- 
posed end-to-end trainable deep networks to directly predict the dense displacement field for image alignment. Wang and Zhang (2020) introduced DeepFLASH for efficient deformable medical image registration, which is implemented in a low dimensional bandlimited space thus dramatically reduces the computational and memory request. To simultaneously enhance the topology preservation and smoothness of the transformation model, Mok and Chung (2020) proposed an efficient unsupervised symmetric image registration method which maximizes the similarity between images within the space of diffeomorphic maps and estimates both forward and inverse transformations simultaneously. In Truong et al. (2020), the authors introduced a universal network for geometric matching, optical flow estimation and semantic corresponding, which can achieve both high accuracy and robustness by investigating the combined use of global and local correlation layers. See more details in the registration-specific reviews (Ferrante and Paragios 2017; Haskins et al. 2020).

Stereo matching. Over the past years, analogous to registration, numerous studies in stereo matching have focused on accurately computing the matching cost by using deep convolutional techniques and refining the disparity map (Zbontar and LeCun 2015; Luo et al. 2016; Zbontar and LeCun 2016; Shaked and Wolf 2017). In addition to the deep descriptors, such as DeepCompare (Zagoruyko and Komodakis 2015) and MatchNet (Han et al. 2015), etc., Zbontar and LeCun (2015) introduced a deep Siamese network to compute the matching cost, which is trained to predict the similarity between image patches. They further proposed a series of CNNs (Zbontar and LeCun 2016) for the binary classification of pairwise matching and applied these in disparity estimation. Similar to converting the computation of matching costs into a multi-label classification problem, Luo et al. (2016) proposed an efficient Siamese network for fast stereo matching. In addition, Shaked and Wolf (2017) improved the performance by computing the matching cost with the proposed constant highway networks and the disparity estimation with reflective confidence learning.

The end-to-end deep manner for this matching task has drawn increasing attention in recent years. For instance, Mayer et al. (2016) trained an end-to-end CNN in their DispNet to obtain a fine disparity map, which is extended by Pang et al. (2017) with a two-stage CNN called cascade residual learning (CRL). More recently, a spatial pyramid pooling module together with a 3-D convolutional strategy has been introduced in Chang and Chen (2018). This approach can exploit global context information to enhance stereo matching. Inspired from CycleGAN (Zhu et al. 2017) and to deal with domain gap, Liu et al. (2020) proposed an end-to-end training framework to translate all synthetic stereo images into realistic ones simultaneously maintain epipolar constraints. This method is implemented through a jointly optimizing between domain translation and stereo matching. Another method in Yang et al. (2020) learns the wavelet coefficients of the disparity rather than the disparity itself, which can learn global context information from low frequency submodule and details from others. Moreover, the guided strategy (Zhang et al. 2019a; Poggi et al. 2019) is also utilized for stereo matching.

Stereo matching with deep convolutional techniques has been dominated for their top performance in public benchmarks $^{2}$. However, the use of CNNs in stereo matching community is limited by the input image pairs, which are generally captured from the binocular camera with a narrow baseline and epipolar rectification. Nevertheless, the network structure, basic ideas, and some tricks or strategies in these learning-based stereo matching may have a strong reference for general image matching tasks.

\subsubsection{Learning from Points}

Learning from points is not as popular as those in images for feature extraction, representation and similarity measurements. Point-based learning, particularly for feature matching, has only been introduced in recent years. This is because using CNNs on point data is more difficult than on raw images due to the unordered structure and dispersed nature of sparse points. Moreover, operating and extracting the spatial relationships, such as neighboring elements, relative positions, length, and angle information, among multi-points using deep convolutional techniques are challenging. However, using deep learning techniques to solve points-based tasks has received increasing considerations. These techniques can be roughly divided into parameter fitting (Brachmann et al. 2017; Ranftl and Koltun 2018) and point classification and/or segmentation (Qi et al. 2017a, b; Moo Yi et al. 2018; Ma et al. 2019a; Zhao et al. 2019). The former is inspired by the classical RANSAC algorithm and aims to estimate the transformation model, such as fundamental matrix (Ranftl and Koltun 2018) and epipolar geometry (Brachmann and Rother 2019), by means of a data-driven optimization strategy with CNNs. However, the latter tends to train a classifier to identify the true matches from putative match set. Generally, parameter fitting and point classification are trained jointly for performance enhancement.

For trainable fundamental matrix estimation, Brachmann et al. (2017) proposed a differentiable RANSAC, termed as DSAC, which is based on reinforcement learning in an endto-end manner. They replaced the deterministic hypothesis selection by probabilistic selection to decrease the expected loss and optimize the learnable parameters. Subsequently, Ranftl and Koltun (2018) presented a trainable method for

\footnotetext{
$\overline{2}$ http://www.cvlibs.net/datasets/kitti/eval_scene_flow.php? benchmark=stereo
} 
fundamental matrix estimation from noise, which is casted as a series of weighted homogeneous least-squares problem, where the robust weights are estimated with deep networks. Similar to DSAC, using learning techniques to improve re-sampling strategy is also introduced in Brachmann and Rother (2019) and Kluger et al. (2020). Brachmann and Rother (2019) proposed NG-RANSAC, a robust estimator using learned guidance of hypothesis sampling. It uses the inlier count itself as training objective to facilitate selfsupervised learning of NG-RANSAC, and can incorporate non-differentiable task loss functions and non-differentiable minimal solvers. While CONSAC (Kluger et al. 2020) is introduced as a robust estimator for multiple parametric model fitting. It uses neural network to sequentially update the conditional sampling probabilities for the hypothesis selection.

Learning-based mismatch removal methods have been developed in recent years. Moo Yi et al. (2018) first attempted to introduce a learning-based technique termed as learning to find good correspondences (LFGC), which aims to train a network from a set of sparse putative matches together with the image intrinsics under the rigid geometrical transformation constraints, and to label the test correspondences as inliers or outliers and output the camera motion simultaneously. However, the LFGC may sacrifice many true correspondences to estimate the motion parameters, failing to handle general matching problems, such as deformable and non-rigid image matching. To this end, Ma et al. (2019a) proposed a general framework to learn a two-class classifier for mismatch removal called LMR, which uses a few images, and handcrafted geometrical representation for training. Their method showed promising matching performance with linearithmic time complexity. More recently, Zhang et al. (2019b) focused on the geometrical recovery based on their order-aware networks (OAN) and have achieved promising performance on pose estimation. Sarlin et al. (2020) proposed SuperGlue, to match two sets of local features by jointly finding correspondences and rejecting non-matchable points. This method is implemented with graph neural networks (Scarselli et al. 2009) for differentiable transport problem optimization. Similar graph neural network pipeline has been adopted by an emerging research branch namely deep graph matching (Wang et al. 2019; Yu et al. 2020a; Fey et al. 2020), where cross-graph convolution (Wang et al. 2019), channelindependent embedding (Yu et al. 2020a) and Spline-based convolution (Fey et al. 2020) are proposed and adopted for supervised graph correspondence learning.

Even though applying CNNs onto point data is difficult, the latest techniques have shown great potential for matrix estimation and point data classification with deep regressor and classifier, particularly for the challenging data or scenarios. Moreover, the multi-layer perception methods in natural language processing and the graph convolutional techniques may serve as great references for addressing these dispersed and unstructured point data in the matching task.

\subsection{Matching in 3-D Cases}

Similar to its 2-D counterpart, 3-D matching methods often involve two steps, i.e., namely, keypoint detection and local feature description, and a sparse correspondence set can then be established by calculating the similarities between descriptors. Although most methods use local feature descriptors, which are designed to be robust to noise and deformations to establish correspondences between 3-D instances, a variety of classical and recent works fall into another category. We refer the readers to the recent surveys (Biasotti et al. 2016; Van Kaick et al. 2011) in the shape matching area given that a detailed review of the literature is beyond the scope for this paper.

The embedding methods aim to parametrize the complex matching problem with less degrees of freedom for tractability by exploiting some natural assumptions (e.g., approximate isometry). A traditional approach is proposed by Elad and Kimmel (2003) to match shapes by embedding them in an intermediate Euclidean space. In this approach, the geodesic distances are approximated by Euclidean ones, and the original non-rigid registration problem is reduced to rigid registration in the intermediate space. Notably, another work developed conformal mapping approaches that also use embedding space (Lipman and Funkhouser 2009; Kim et al. 2011; Zeng et al. 2010).

A more direct approach is to find a point-wise matching between (subsets of) points on shapes by minimizing the structure distortion. This formulation was developed by Bronstein et al. (2006), who introduced a highly nonconvex and non-differentiable objective and generalized multidimensional scaling technique for optimization. Some researchers have also attempted to mitigate the prohibitively high computational complexity issue (Sahillioglu and Yemez 2011; Tevs et al. 2011) while considering the quadratic assignment formulation (Rodola et al. 2012, 2013; Chen and Koltun 2015; Wang et al. 2011) in graph matching.

The family of methods based on the functional map framework was first developed by Ovsjanikov et al. (2012). Instead of point-to-point matching in Euclidean space, these methods represent the correspondences using the functional map between two manifolds, which can be characterized by linear operators. The functional map can be encoded in a compact form by using the eigenbases of the Laplace-Beltrami operator. Most natural constraints on the map, such as landmark correspondences and operator commutativity, become linear in this formulation, leading to an efficient solution. This approach was adopted and extended in many follow-up works (Aflalo et al. 2016; Kovnatsky et al. 2015; Pokrass et al. 2013; Rodolà et al. 2017; Litany et al. 2017). 
Point set learning in 3-D cases for registration is also a hot topic. Yew et al. (2020) proposed RPM-Net for rigid point cloud registration, in which it desensitizes initialization and improves convergence performance with learned fusion features. Gojcic et al. (2020) introduced an end-toend multiview point cloud registration framework by directly learning to register all views of a scene in a globally consistent manner. Pais et al. (2020) introduced a learning architecture for 3D point registration, namely 3DRegNet. This method can identify true point correspondences from a set of putative matches, and regress the motion parameters to align the scans into a common reference frame. Choy et al. (2020) used high-dimensional convolutional networks to detect linear subspaces in high-dimensional spaces, then applied it for $3 \mathrm{D}$ registration under rigid motions and image correspondence estimation.

\subsection{Summary}

Given a pair of images of similar object/scene and with/without the feature detection and/or description, the matching tasks have been extended into several different forms, such as image registration, stereo matching, feature matching, graph matching, and point set registration. These different matching definitions are generally introduced for specific applications, with their own strengths presented.

Traditional image registration and stereo achieve dense matching by means of patch-wise similarity measuring together with optimization strategy to search the overall optimal solution. However, they are conducted on image pairs of high overlapping area (slight geometrical deformation) and binocular camera, and these may require large computational burden and the limited handcrafted measuring metrics.

The introduction of deep learning has promoted registration accuracy and disparity estimation due to advancements in network design and loss definition, as well as abundant training samples. However, we also find that using deep learning for these matching tasks is usually performed on image pairs undergoing slight geometrical deformation such as medical image registration and binocular stereo matching. Applying them for more complex scenarios, such as wide baseline images stereo or image registration with serious geometric deformations, still remains open.

Feature-based matching can effectively address the limitations in large viewpoint, wide baseline, and serious non-rigid image matching problems. Among those proposed in the literature, the most popular strategy is to construct the putative matches based on descriptor distance, followed by a robust estimator such as RANSAC. However, a large number of mismatches in the putative match sets may negatively affect the performance in subsequent visual task and also require considerable time for model estimation. Therefore, the mismatch removal method is required and integrated to preserve as many true matches as possible while maintaining the mismatch to a minimum level using extra geometrical constraints. Specifically, the resampling-based method, such as RANSAC, can estimate the latent parameter model and simultaneously remove the outliers. However, their theoretically required runtime grows exponentially with the increase in outlier rate, and they cannot process the image pairs that undergo more complex non-rigid transformations. The nonparametric model-based methods can handle the non-rigid image matching problem by using high-dimensional nonparametric model, but it is still challenging in defining the objective function and finding the optimal solution in a more complex solution space. Different from the global constraints in the resampling and non-parameter model-based methods, the relaxed mismatch removal methods are commonly conducted on a local coherent assumption of potential inliers. Thus, much simpler but efficient rules are designed to filter out the outliers while maintaining the inliers within an extremely short time. However, methods of this type are limited due to their parameter sensitivity; moreover, they are prone to preserve evident outliers, thereby affecting the accuracy of subsequent pose estimation and image registration.

In addition, the image patch-based descriptor may not be workable due to the matching request in less-texture images, shape, semantic images, and the raw points directly captured from specific device. Therefore, for performing the matching task of these situations, the graph matching and point registration methods are more suitable. The graph structure among neighboring points and the overall corresponding matrix are applied to optimize and find the optimal solution. However, these pure point-based methods are limited by restrictions in their computation burden and outlier sensitivity. Therefore, designing appropriate problem formulation and constraint conditions, and proposing more efficient optimization methods, are still open problems in image matching community and require further research attention.

Analogously to image-based learning, increasing studies have used deep learning in feature-based matching community. The latest techniques have shown great potential for matrix estimation (e.g. fundamental matrix) and point data classification (such as mismatch removal) with deep regressor and classifier, particularly for handling challenging data or scenarios. However, conducting convolutional networks on point data is not as easy as on raw images due to the unordered structure and dispersed nature of these sparse points. Nevertheless, recent studies have shown the feasibility of using the graph convolutional strategy and multi-layer perception methods, together with specific normalization on such point data. In addition to rigid transformation parameter estimation, matching on point data with non-rigid and even serious deformation by using deep convolutional techniques may be a more challenging and significant problem. 


\section{Matching-Based Applications}

Image matching is a fundamental problem in computer vision and is considered a critical prerequisite in a wide range of applications. In this section, we briefly review several representative applications.

\subsection{Structure-from-Motion}

Structure-from-motion (SfM) involves recovering the 3-D structure of a stationary scene from a series of images, which are obtained from different viewpoints by estimating the camera motions corresponding to these images. SfM involves three main stages, namely, (i) feature matching across images, (ii) camera pose estimation, and (iii) recovery of the 3-D structure using the estimated motion and features. Its efficacy largely depends on the admissible set of feature matches.

In modern SfM systems (Schonberger and Frahm 2016; Wu 2018; Sweeney et al. 2015), the feature matching pipeline is widely adopted across images, i.e., feature detection, description, and nearest-neighbor matching, to provide initial correspondences. The initial correspondences contain a number of outliers. Thus, geometric verification is required, which is tackled via the estimation fundamental matrix using RANSAC (Fischler and Bolles 1981). This can potentially be addressed by mismatch removal methods.

Meanwhile, to enhance the SfM task, researchers have focused on performing robust feature matching, i.e., thus establishing rich and accurate correspondences. Evidently, advanced descriptors can greatly affect this task (Fan et al. 2019). Moreover, Shah et al. (2015) proposed a geometryaware approach, which initially uses a small sample of features to estimate the epipolar geometry between the images and leverages it for the guided matching of the remaining features. Lin et al. (2016b) utilized RANSAC to guide the training of match consistency curves for differentiating true and false matches. Their approach traces the common problems of wide-baselines and repeated structures for reconstructing modern cities. These correspondences are also the prerequisites for camera pose estimation, and the effective substitution of commonly used RANSAC for this task has also been investigated (Moo Yi et al. 2018), with a pre-stage of identifying good correspondences.

\subsection{Simultaneous Localization and Mapping}

Acquiring maps of the environment is a fundamental task for autonomous mobile robots, thereby forming the basis of many different higher-level tasks, such as navigation and localization. The problem of simultaneous localization and mapping (SLAM) (Davison et al. 2007; Mur-Artal et al. 2015;
Sturm et al. 2012) has received intensive attention over the decades.

In common SLAM systems, feature matching is needed to establish correspondences between frames, which then serve as the input for estimating the relative camera pose and localization. Similar to SfM, the full-fledged feature matching pipeline is used in most SLAM systems. Typically, in Endres et al. (2012), Endres et al. introduced a SLAM system that incorporates feature matching to establish spatial relations from the sensor data in the front-end. The well-known SIFT (Lowe 2004), SURF (Bay et al. 2008), and ORB (Rublee et al. 2011) algorithms are optionally used to detect and describe features, and RANSAC (Fischler and Bolles 1981) is subsequently used for robust matching.

An evaluation of different feature detectors and descriptors can be found in Gil et al. (2010). Recently, Lowry and Andreasson (2018) proposed a spatial verification method for visual localization, which is robust in the presence of a high proportion of outliers. For a SLAM system that percepts 3$\mathrm{D}$ range scans, the point set registration methods (e.g. ICP) (Nüchter et al. 2007) are also used for scan matching and localizing the robot.

Loop closure detection-another core module in SLAM application-refers to accurately asserting that an agent has returned to a previously visited location. It is crucial to reduce the drift of the estimated trajectory caused by accumulative error. A group of appearance-based approaches have been developed to use image similarities to identify previously visited places. Feature matching results are naturally applicable to measure the similarity of two scenes and have been the bases of many state-of-the-art methods. For example, Liu and Zhang (2012) performed feature matching with SIFT between the current image and each previously visited image, after which they determined the closed loop on the basis of the number of accurate matches in the results. Zhang et al. (2011) used directed matching of raw features extracted from images for detecting loop-closure events. To achieve loop closure detection, Wu et al. (2014) used LSH as the basic technique by matching the binary visual features in the current view of a robot with the visual features in the robot appearance map. Liu et al. (2015a) developed a consensus constraint to prune outliers and verified the superiority of their methods for loop closure detection.

\subsection{Visual Homing}

Visual homing aims to navigate a robot from an arbitrary starting position to a goal or home position based solely on visual information. This is often accomplished by estimating a homing vector/direction (pointing from the current position to the home position) from two panoramic images, which are captured respectively at the current position and the home position. Conventionally, feature matching serves 
as the building block of correspondence methods in visual homing research (Möller et al. 2010). In this category, the homing vector can be determined by transforming the correspondences into motion flows (Ma et al. 2018b; Churchill and Vardy 2013; Liu et al. 2013; Zhao and Ma 2017).

Ramisa et al. (2011) combined the average landmark vector with invariant feature points automatically detected in panoramic images to achieve autonomous visual homing. However, the feature matches are solely determined by the similarity of the descriptors in the method, thus leading to a number of mismatches. The presence of outliers has been verified to be the reason of performance degradation for visual homing (Schroeter and Newman 2008). In order to resolve the degradation caused by mismatches, Liu et al. (2013) used a RANSAC-like method to remove mismatches. Meanwhile, Zhao and Ma (2017) proposed a visual homing method by simultaneously mismatch removal and robust interpolation of sparse motion flows under a smoothness prior. Ma et al. (2018b) also proposed a guided locality preserving matching method to handle extremely large proportions of outliers and improve the visual homing robustness.

\subsection{Image Registration and Stitching}

Image registration is the process of aligning two or more images of the same scene obtained from different viewpoints, at different times, or from different sensors (Zitova and Flusser 2003). In the past decades, feature-based methods in which the key requirement is feature matching have gained increasing attention due to its robustness and efficiency. Once the correspondence is established, image registration is reduced to estimate the transformation model (e.g., rigid, affine, or projective). Finally, the source image is transformed by means of the mapping functions, which rely on some interpolation technique (e.g., bilinear and nearest neighbor). A large number of works have been proposed for feature matching and image registration. Ma et al. (2015b) proposed a Bayesian formulation for rigid and non-rigid feature matching and image registration. To further exploit the geometrical cues, the locally linear transforming constraint is incorporated. They also recently proposed a guided locality preserving matching method (Ma et al. 2018a). Their proposed method can significantly reduce the computational complexity and is able to deal with a more complex transformation model. For non-rigid image registration, Pilet et al. (2008) and Gay-Bellile et al. (2008) proposed solutions, where robust matching techniques are insensitive to outliers. Some efforts (Paul and Pati 2016; Ma et al. 2017b; Yang et al. 2017a) also attempted to modify feature detectors and descriptors to improve the registration process.

The problem of multi-modal image registration is more complicated due to the high variability of appearance caused by different modalities, which frequently arise in medical image and multi-sensor image analysis. For example, Chen et al. (2010) developed the partial intensity invariant feature descriptor (PIIFD) to register retinal images, whereas Wang et al. (2015) extended PIIFD in a more robust registration framework with SURF detector (Bay et al. 2008) and a single Gaussian point matching model. On the basis of the characteristics of multi-modal images, Liu et al. (2018a) proposed an affine and contrast invariant descriptor for IR and visible image registration. Du et al. (2018) also proposed an IR and visible image registration method based on scale-invariant PIIFD feature and locality preserving matching. Ye et al. (2017) proposed a novel feature descriptor based on the structural properties of images for multi-modal registration. A detailed discussion of feature matching-based, multi-modal registration techniques of the medical image analysis area, which are categorized as geometric methods, can be found in Sotiras et al. (2013).

Meanwhile, image stitching or image mosaic involves obtaining a wider field-of-view of a scene from a sequence of partial views (Ghosh and Kaabouch 2016). Compared to image registration, image stitching deals with low overlapping images and requires accurate alignment in the pixellevel to avoid visual discontinuities. Feature-based stitching methods are popular in this area because of their invariance properties and efficiency. For example, in order to identify geometrically consistent feature matches and achieve accurate homography estimation, Brown and Lowe (2007) proposed the use of the SIFT (Lowe 2004) feature matching and the RANSAC (Fischler and Bolles 1981) algorithm. Lin et al. (2011) used SIFT (Lowe 2004) to pre-compute matches and then jointly estimating the matching and the smoothly varying affine fields for better stitching performance. Interested readers can refer to the comprehensive survey (Ghosh and Kaabouch 2016; Bonny and Uddin 2016) for an overview of more feature-based image mosaic and stitching methods.

\subsection{Image Fusion}

To generate a more conducive image to subsequent applications, image fusion is adopted to combine the meaningful information from images acquired by different sensors or under different shooting settings (Pohl and Van Genderen 1998), wherein the source images have been accurately aligned in advance. The very premise of image fusion is to register source images using feature matching methods, and the accuracy of registration directly affects the fusion quality. Liu et al. (2017) used the CNN to jointly generate the activity level measurement and fusion rules for multi-focus image fusion. Meanwhile, Ma et al. (2019c) proposed an end-to-end model for infrared and visible image fusion, which generates images with a dominant infrared intensity and an additional visible gradient under the framework of generative adversarial networks. Subsequently, they introduced a detail loss and 
a target edge-enhancement loss to further enrich the texture details (Ma et al. 2020).

A group of methods aim to fuse images based on the local features, among which the dense SIFT is the most popular. Liu et al. (2015b) proposed the fusion of multi-focus images with dense scale invariant feature transform, wherein the local feature descriptors are used not only as the activity level measurement, but also to match the mis-registered pixels between multiple source images to improve the quality of the fusion results. Similarly, Hayat and Imran (2019) proposed a ghost-free multi-exposure image fusion technique using the dense SIFT descriptor with a guided filter, which can produce high-quality images using ordinary cameras. In addition, Chen et al. (2015) and Ma et al. (2016a) introduced a method that can perform image registration and image fusion simultaneously, thus fulfilling image fusion on unaligned image pairs.

\subsection{Image Retrieval, Object Recognition and Tracking}

Feature matching can be used to measure similarity between images, thereby enabling a series of high-level applications, including image retrieval (Zhou et al. 2017), object recognition, and tracking. The goal of image retrieval is to retrieve all images that exhibit similar scenes for a given query image. In local feature-based image retrieval, the image similarity is intrinsically determined by the feature matches between images. Thus, the image similarity score can be obtained by aggregating votes from the matched features. In Zhou et al. (2011), the relevance score is simply determined by the number of feature matches across two images. In Jégou et al. (2010), the scoring function is defined as a cumulation of the squared term frequency inverse document frequency weights on shared visual words, which is essentially a bag of features of inner products.

Moreover, geometric context verification, a common technique for refining initial image retrieval result, is directly related to feature matching. By incorporating the geometrical information, geometric context verification technique can be used to address the false match problem caused by the ambiguity of local descriptor and the quantization loss. For image retrieval, a large group of methods estimate the transformation model in an explicit approach to verify the tentative matches. For example, Philbin et al. (2007) used a RANSAClike method to find the inlier correspondences, whereas Avrithis and Tolias (2014) developed a simple spatial matching model inspired by Hough voting in the transformation space. Another line of works address geometric context verification without explicitly handling a transformation model. For example, Sivic and Zisserman (2003) utilized the consistency of spatial context in local feature groups to verify the tentative correspondences. Zhou et al. (2010) proposed the spatial coding method, whereby the valid visual word matches are identified by verifying the global relative position consistency.

With the function of measuring similarity, feature matching also plays an important role in object recognition and tracking. For example, Lowe et al. (1999) used SIFT features to match sample images and new images. In their proposed method, the potential model pose is identified through a Hough transform hash table and then through a least-squares fit to achieve a final estimate of model parameters. The presence of the object is strongly evident if at least three keys agree on the model parameters with low residuals. Modern attempts for object recognition also include some specifically handcrafted features (Dalal and Triggs 2005; Hinterstoisser et al. 2012) and, more recently, deep learning approaches (Wohlhart and Lepetit 2015).

Tracking basically refers to estimating the trajectory of an object over images. Feature matching across images is the basis of feature-based tracking, and a variety of algorithms for these tasks have been proposed in the literature. The feature matching pipeline is adopted in most visual tracking systems, except that the matching is constrained to those of the known features that are predicted to lie close to the encountered position. The readers are referred to a comprehensive evaluation of different feature detectors and descriptors for tracking by Gauglitz et al. (2011), and the recently presented benchmark (Wu et al. 2015b), which covers a review of modern object tracking methods as well as the role played by feature representation methods.

\section{Experiments}

Diverse methods for image matching have been proposed, particularly when the deep learning techniques are becoming increasingly popular. However, the question of which method would be suitable for specific applications under different scenarios and requirements still remains. We are encouraged to conduct more comprehensive and objective comparative analysis of these classical and state-of-the-art techniques.

\subsection{Overview of Existing Reviews}

To evaluate the existing matching methods at an early time, the classical image registration survey (Zitova and Flusser 2003) provided several definitions for evaluation of registration accuracy including localization error, matching error, and alignment or registration error. In 2005, Mikolajczyk et al. evaluated affine region detectors (Mikolajczyk et al. 2005) and local descriptors (Mikolajczyk and Schmid 2005) against changes of viewpoint, scale, illumination, blur, and image compression on their own proposed VGG (a.k.a. Oxford) datasets. They also presented a comprehensive compari- 

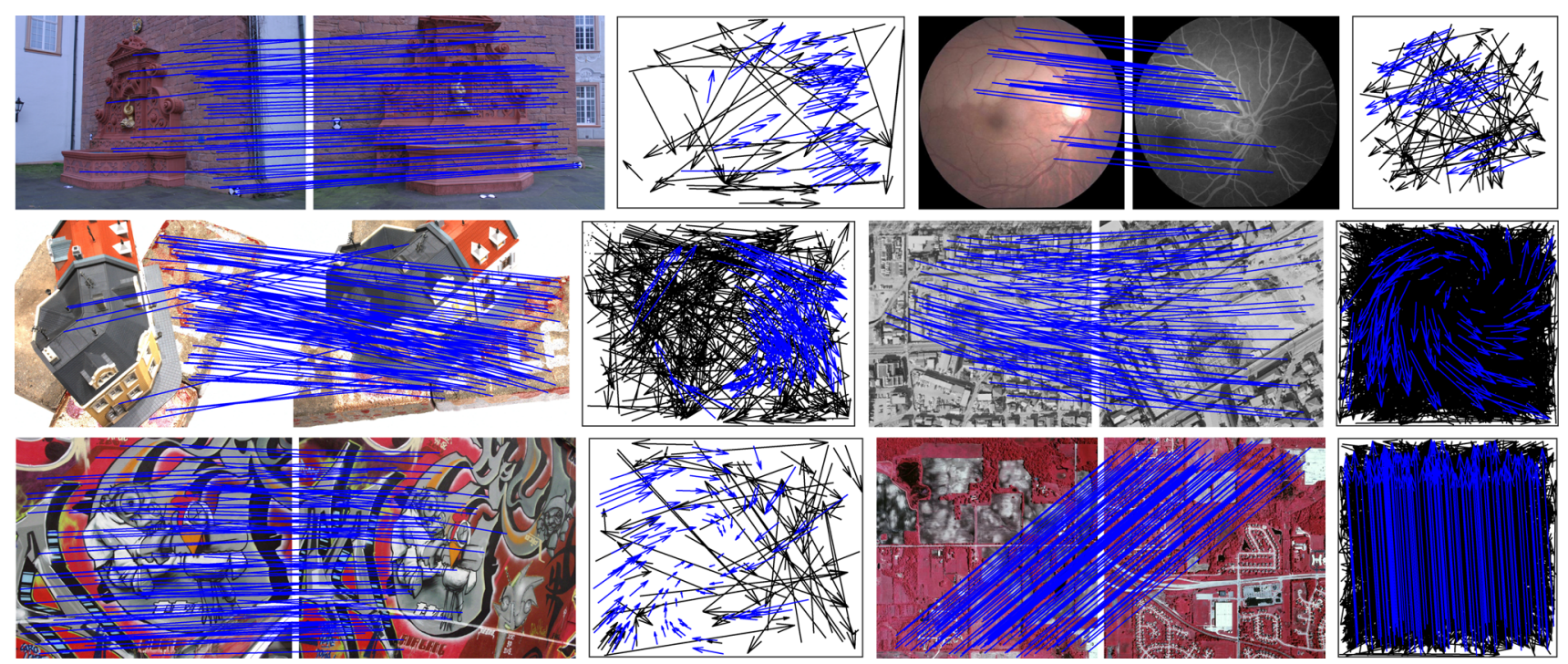

Fig. 2 Examples of the five datasets. The ground truth is given using colored correspondences. The head and tail of each arrow in the motion field correspond to the positions of feature points in two images (blue
$=$ true positive, black $=$ true negative). For visibility, in the image pairs, at most 100 randomly selected matches are presented, and the true negatives are not shown (Color figure online) son on repeatability and accuracy for detectors and recall, 1 - precision for descriptors. Subsequently, Strecha et al. (2008) published a dense 3-D dataset for wide-baseline stereo and 3-D geometrical and camera pose evaluation.

In addition, Aanæs et al. (2012) evaluated some representative detectors using a large dataset of known camera positions, controlled illumination, and 3-D models, namely, DTU. At the same time, Heinly et al. (2012) compared the traditional float and binary feature operators in 2012 and evaluated their matching performance with the inter-combination of existing detectors and descriptors on the public and their own datasets. The evaluation was conducted on more systematic performance metrics consisting of putative match ratio, precision, matching score, recall, and entropy. Similarly, using inter-combination strategy, Mukherjee et al. (2015) provided a comparative experimental analysis for selecting appropriate combination of various detectors and descriptors in order to solve the problems of image matching using different image data.

More recently, inspired by emerging deep learning techniques, Balntas et al. (2017) reported that existing defective datasets and evaluation metrics may lead to unreliable comparative results. Thus, they proposed and publicized a large benchmark for handcrafted and learned local image descriptors called Hpathes. They also comprehensively evaluated the performance of widely used handcrafted descriptors and recent deep ones with extensive experiments on patch recognition, patch verification, image matching, and patch retrieval. Schonberger et al. (2017) conducted an experimental evaluation of learned local features, including classical machine learning based variants of SIFT and recent CNN- based techniques, in which they considered that finding additional true matches between similar images does not necessarily improve performance when matching images under extreme viewpoint or illumination changes. Mitra et al. (2018) provided a PhotoSynth (PS) dataset for training local image descriptors. Komorowski et al. (2018) provided a stability evaluation for handcrafted and learning-based interest point detectors on ApolloScape street dataset (Huang et al. 2018). A comprehensive comparison of local image feature detectors based on both classical and CNN techniques is conducted on public datasets (Lenc and Vedaldi 2014). That work proposed a modified repeatability for detection evaluation, which is more robust to feature scale variety. Jin et al. (2020) introduced a benchmark for local features and robust estimation algorithms, focusing on the accuracy of the reconstructed camera pose as their practical evaluation. In addition, Bellavia and Colombo (2020) provided a comprehensive analysis and evaluation about the descriptor design based on SIFT.

From the above mentioned, we can know that several comprehensive and thorough evaluation of feature detectors and descriptors can be found in Komorowski et al. (2018), Lenc and Vedaldi (2014), Heinly et al. (2012) and Schonberger et al. (2017). However, in order to evaluate the local feature methods, many studies compared the matching performances on a 3-D reconstruction task, including the works of Fan et al. (2019) and Schonberger et al. (2017). In the 3-D case, Tombari et al. (2013) presented a thorough evaluation of several state-of-the-art 3-D keypoint detectors, and Guo et al. (2016) compared ten popular local feature descriptors in the contexts of 3-D object recognition, 3-D shape retrieval, and 

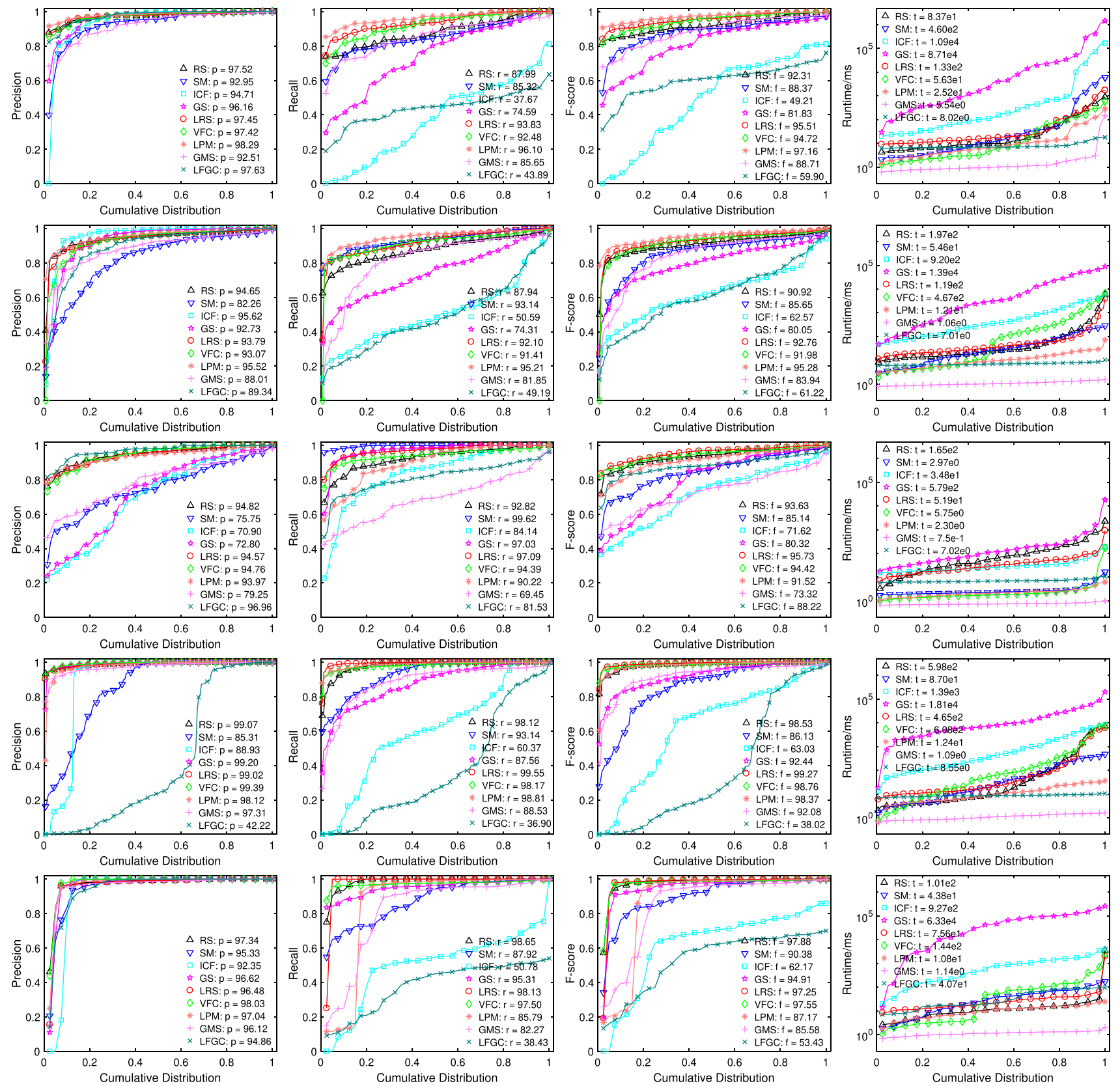

Fig. 3 Quantitative performance of the state-of-the-art mismatch removal algorithms on the introduced five datasets. The statistics of precision, recall, F-score and runtime are reported for each dataset, and the average values are given in the legend. From top to bottom, the statistics of DAISY, DTU, Retina, RemoteSensing and VGG. The results are

presented in cumulative distribution, a point on the curve with coordinate $(\mathrm{x}, \mathrm{y})$ denotes that there are $(100 * x)$ percents of image pairs which have the performance value (i.e., precision, recall, F-score or runtime) no more than $y$

3-D modeling. Several matching related applications, such as image retrieval (Zheng et al. 2018) and visual localization (Piasco et al. 2018), have also been evaluated recently. We refer the readers to these works for a detailed discussion of their performance. For mismatch removal, point set registration, graph matching, and the application performance of pose estimation and loop-closure detection, we will present both quantitative and qualitative comparisons.

\subsection{Results on Mismatch Removal}

We conduct experiments on five image matching datasets with ground truth. Our primary aim is to evaluate different 
mismatch removal methods. The features of each image are assumed to be detected and described, and the open source VLFeat toolbox is used to determine the putative correspondence using SIFT (Lowe 2004). The details of the adopted datasets are described as follows, and some representative image matching examples from the used datasets are illustrated in Fig. 2. The ground truth of each dataset is checked by the provided geometrical transform matrix, such as homograph, or provided in the manner that each match is manually labeled as true or false. The experiments of this part are performed on a desktop with $3.4 \mathrm{GHz}$ Intel Core i5-7500 CPU, 8GB memory.

DAISY (Tola et al. 2010): The dataset consists of wide baseline image pairs with ground truth depth maps, including two short image sequences and several individual image pairs. We match each two images in one sequence and all the individual pairs are used, which creates in total 47 image pairs for evaluation. This dataset is a challenging one due to the large number of matches, which is up to 8000 . The average numbers of matches and inlier rate are 1191.6 and $77.99 \%$, respectively.

DTU (Aanæs et al. 2016): The dataset is originally designated for multiple-view stereo evaluation, which involves a number of different scenes with a wide range of objects. The ground truth camera positions and internal camera parameters have high accuracy. Two scenes are selected for this dataset (i.e., Frustum and House), after which we create 130 image pairs for evaluation. These scenes generally have large viewpoint changes in the scenes. The average numbers of matches and inlier rate are 729.3 and $58.83 \%$, respectively.

Retina (Ma et al. 2019d) It consists of 70 retinal image pairs with non-rigid transformation. Due to different modalities between images, ambiguous putative matches are generated, resulting in a small number of correct matches and a low inlier ratio. The average numbers of matches and inlier rate are 158.4 and $41.56 \%$, respectively.

RemoteSensing (Ma et al. 2019d) There are 161 remote sensing image pairs including color-infrared, SAR, and panchromatic photographs. The feature matching task for such image pairs typically arises in image-based positioning as well as navigating and change detection. The average numbers of matches and inlier rate are 767.6 and $68.50 \%$, respectively.

$V G G$ (Mikolajczyk and Schmid 2005) It contains 40 image pairs either of planar scenes or captured by a camera in a fixed position during acquisition. Hence, the image transformation can be precisely described by homography. The ground truth homographies are included in the dataset.

These abovementioned datasets are collected and available at. ${ }^{3}$ In addition, a small UAV image registration dataset (SUIRD) is also provided for image registration or match-

\footnotetext{
${ }^{3}$ https://github.com/StaRainJ/Imgae_matching_Datasets.
}

ing research. This dataset includes 60 pairs of low-altitude remote sensing images captured by small UAV and their groundtruth. The image pairs contain viewpoint changes in horizontal, vertical, their mixture and extreme patterns which produce problems of low overlap, image distortion and severe outliers. ${ }^{4}$ Throughout the experiments, we use three evaluation metrics: precision, recall, and F-score. Given the number of true positives (TP), true negatives (TN), false positives (FP) and false negatives (FN), the precision is obtained by:

$$
P=\frac{T P}{T P+F P} .
$$

The recall is given as follows:

$$
R=\frac{T P}{T P+F N} .
$$

The F-score, as a summary statistic of precision and recall, is obtained as follows:

$F=\frac{2 \times P \times R}{P+R}$.

The mismatch removal methods include: RANSAC (Fischler and Bolles 1981) (abbreviated as RS), SM (Leordeanu and Hebert 2005), ICF ( $\mathrm{Li}$ and Hu 2010), GS (Liu and Yan 2010), LO-RANSAC (Lebeda et al. 2012) (abbreviated as LRS), VFC (Ma et al. 2014), LPM (Ma et al. 2019d), GMS (Bian et al. 2017), and LFGC (Moo Yi et al. 2018).

Figure 3 shows the performance on the five datasets evaluated by precision, recall, F-score, and runtime with cumulative distribution. In addition, the average values of each statistic is summarized in Table 1 for a more straightforward comparison. The graph matching methods, SM and GS, have shown relatively weak performances given the graphical model, albeit with strong generality, only excavates the shallow pairwise geometric constraints. Random sampling methods, RS and LRS, hold the key assumption that the image pairs are related by parametric models. This assumption seems to work well in the datasets; however, their time costs are not favorable. The non-parametric interpolation method VFC is relatively robust and outperforms ICF. However, its computational cost is higher than that of some other strong competitors, e.g., LPM. LPM is simple to implement. It utilizes a more relaxed geometric constraint, yet it achieves surprisingly excellent performance and becomes the best performer considering the time cost. Compared with GMS, it obtains much better performance with only a slight increase in runtime. The recent trend has suggested a deep learning paradigm for differentiating mismatches, e.g. LFGC. LFGC has proven to be much more effective than the traditional

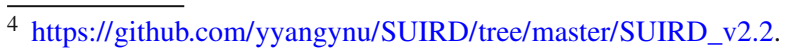




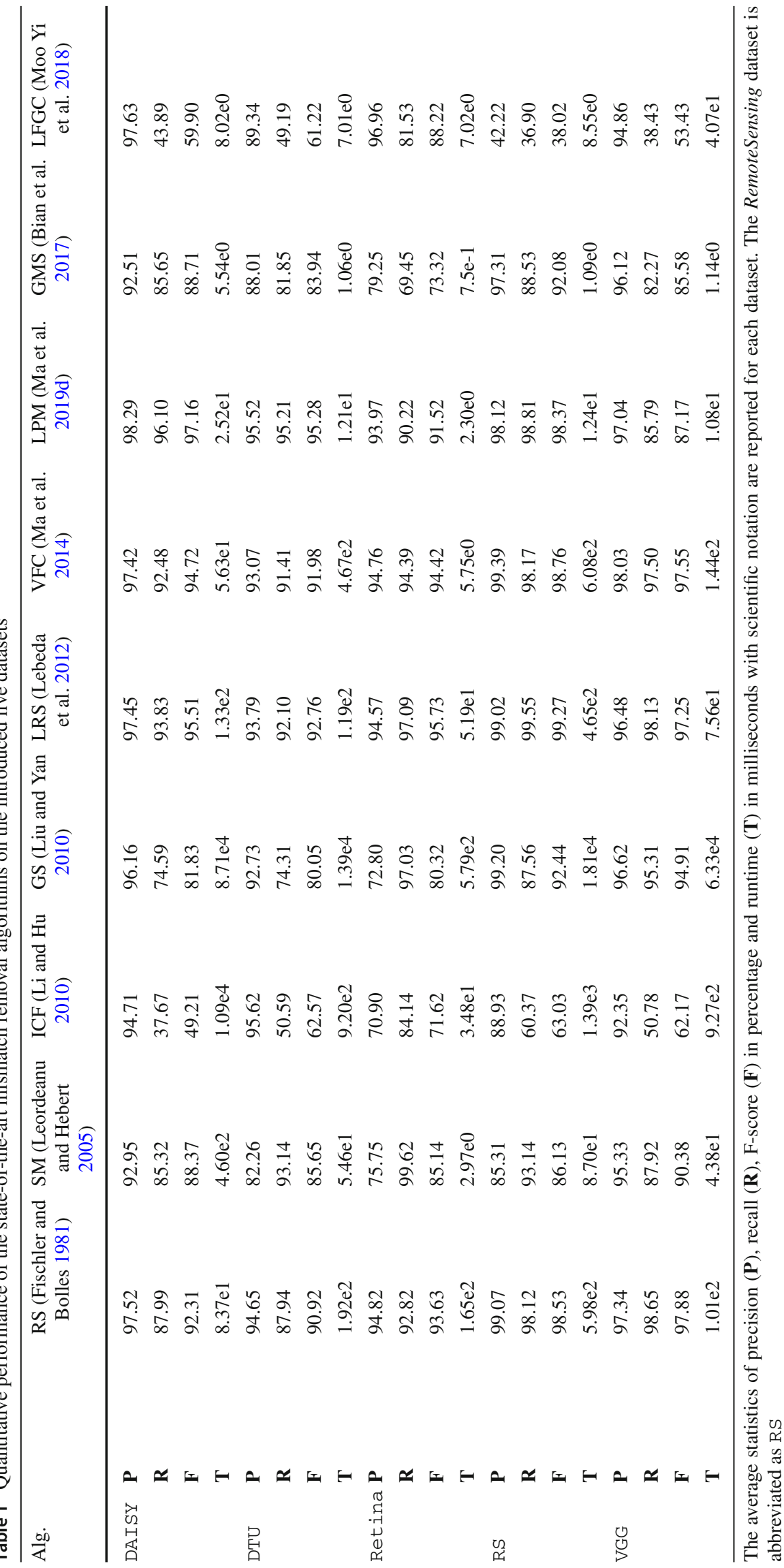



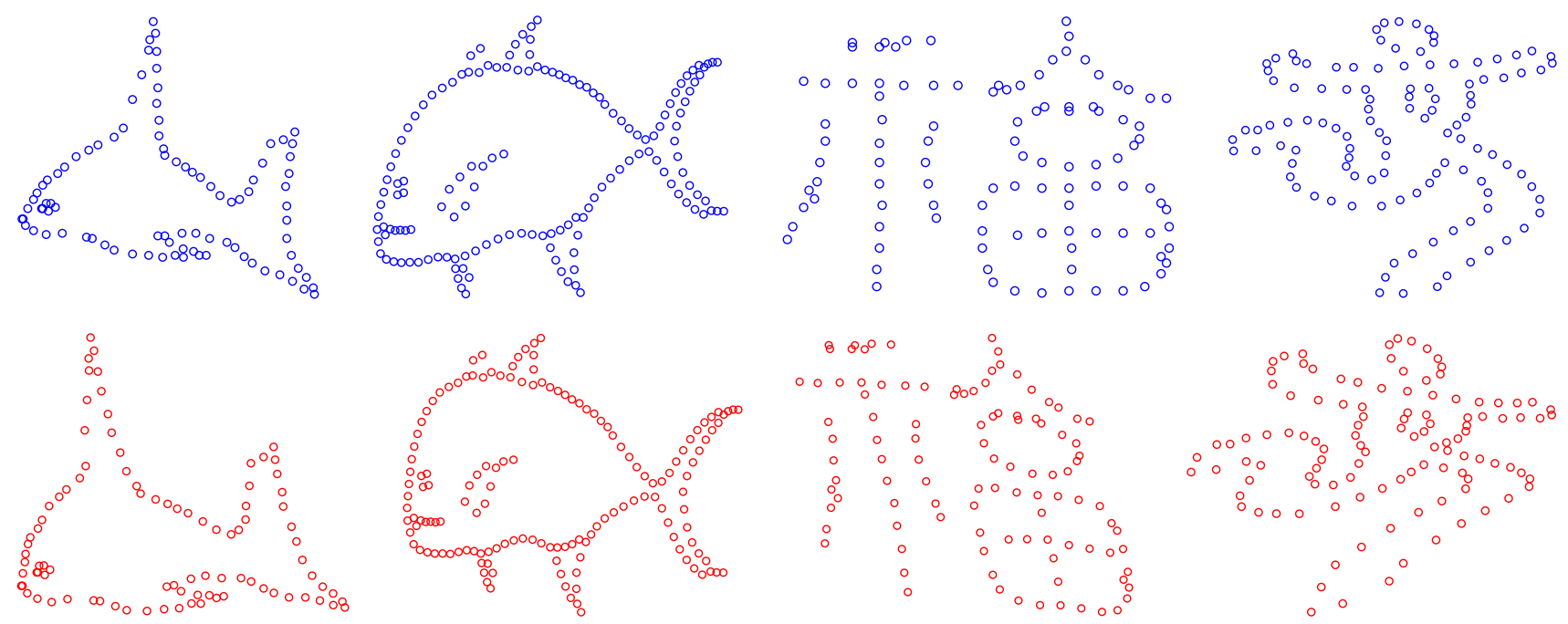

Fig. 4 2-D shape contours used in our experiments, from left to right, fish, whale, fu, beijing
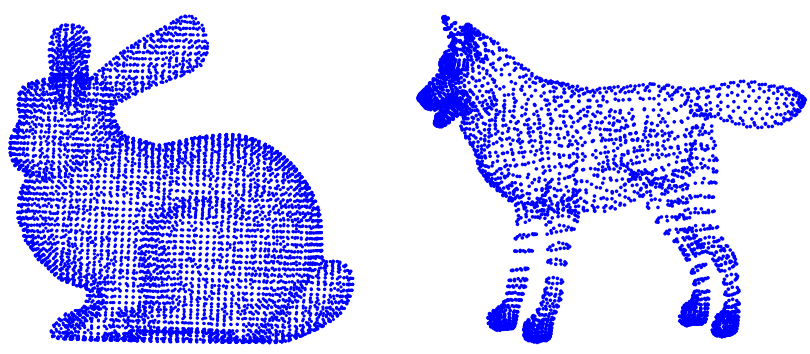

Fig. 5 The bunny and wolf pattern of 3-D point cloud used in our experiments

methods. However, in our case, it has a restricted performance with low recall and high accuracy, resulting in the failure in RemoteSensing. This finding indicates that the learning methods are data-dependent with limited generality.

\subsection{Results on Point Set Registration}

The experiments for point set registration consist of two parts: non-rigid registration with 2-D shape contour data and rigid registration with 3-D point cloud data. In the 2-D case, six representative methods, namely, TPS-RPM (Chui and Rangarajan 2003), GMM (Jian and Vemuri 2011), CPD (Myronenko and Song 2010), $L_{2} E$ (Ma et al. 2013b), PRGLS (Ma et al. 2015a), and APM (Lian et al. 2017) are evaluated. In the 3-D case, the rigid versions of GMM and CPD as well as ICP (Besl and McKay 1992) and GoICP (Yang et al. 2016) are evaluated. The experiments of this part are performed on a desktop with $3.4 \mathrm{GHz}$ Intel Core i5-7500 CPU, 8GB memory.

The point data are normalized as inputs, thus allowing the use of a fixed threshold to evaluate the registration performance. Specifically, a point is accurately aligned if its distance to the ground truth corresponding point is below a given threshold. Thus, we can define the accuracy of registration as the percentage of accurately aligned points. In our experiment, the threshold is empirically set to 0.1 . Four patterns are collected to evaluate the non-rigid 2-D registration results, as shown in Fig. 4. We also create five deformed shapes for each pattern as the data to be registered, generating a total of 20 instances. We also conduct noise, outlier, and rotation experiments on these instances. For the 3-D case, as shown in Fig. 5, two patterns are used, and we exert random rotation to create 20 instances for each pattern. Noise and outlier experiments are also conducted on these 40 instances.

The results of non-rigid 2-D registration are presented in Fig. 6. The outlier experiments of APM are excluded due to its prohibitive runtime with the increase in data points. The experimental setting is relatively challenging, and the weaknesses of each method have emerged. For instance, TPS-RPM is generally robust to outliers, but it can be degraded in the case of severe noises. CPD and GMM have similar performances and are sensitive to outliers. $L_{2} E$ and PR-GLS utilize the information of shape context descriptor to guide the registration, but their performances are unstable. APM can only deal with affine deformation, thus leading to its inferior performance. However, compared to other methods that are only locally convergent and fail to handle violent rotations, APM is invariant to rotation owing to its global optimality.

The results of rigid 3-D point cloud registration are presented in Fig. 7. In our random rotation settings, the locally convergent methods, i.e., GMM, CPD, and ICP, fail to accurately register the point clouds. In this regard, the globally optimal method, GoICP, outperforms them by a large margin. 

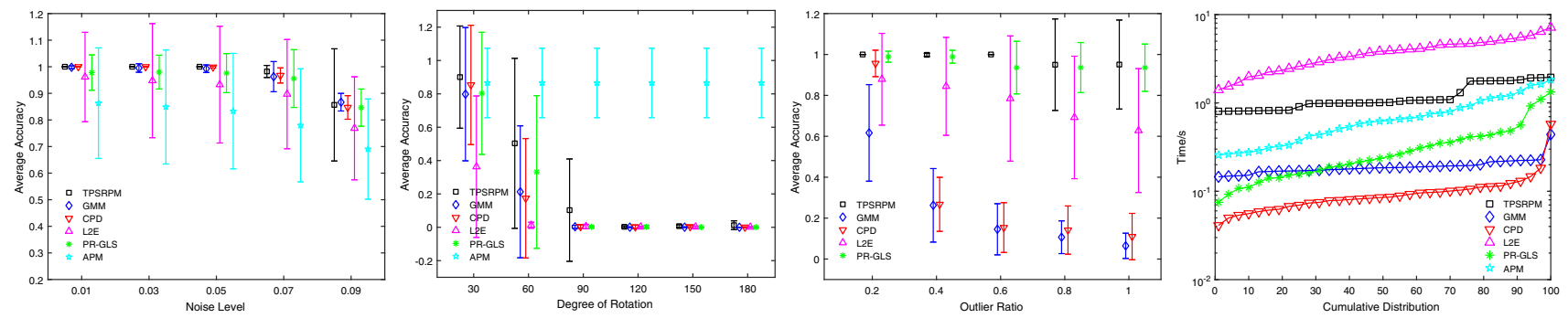

Fig.6 Quantitative evaluation of non-rigid 2-D shape contour registration
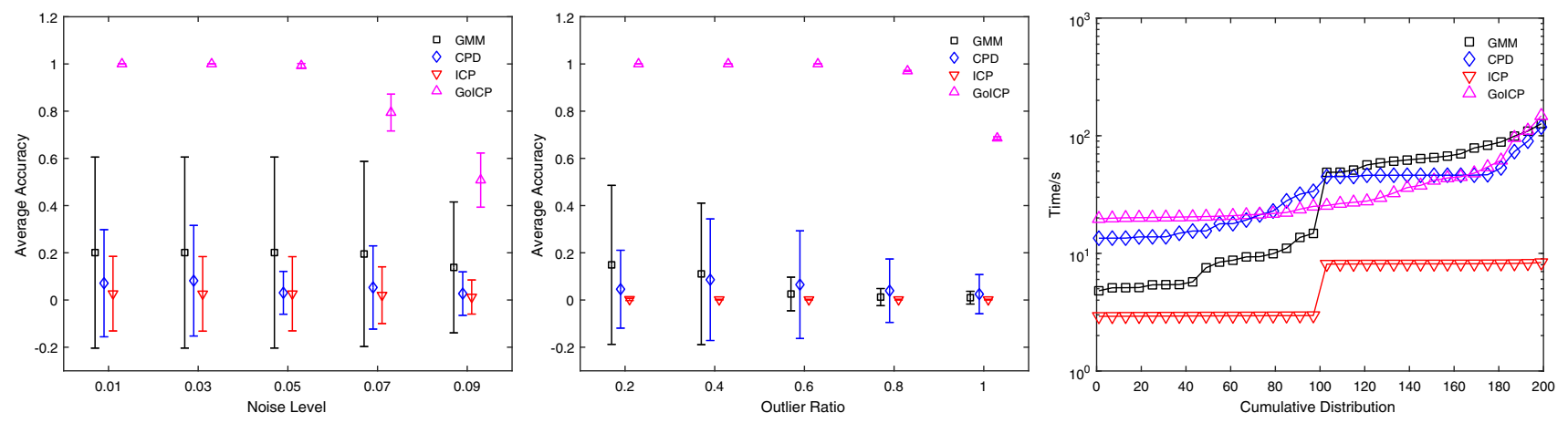

Fig.7 Quantitative evaluation of rigid 3-D point cloud registration

\subsection{Results on Graph Matching}

Graph matching represents an alternative means to establish correspondences between two feature sets. Here, we evaluate seven state-of-the-art methods in the literature, namely, SM (Leordeanu and Hebert 2005), SMAC (Cour et al. 2007), IPFP (Leordeanu et al. 2009), RRWM (Cho et al. 2010), TM (Duchenne et al. 2011), GNCCP (Liu and Qiao 2014), and FGM (Zhou and De la Torre 2015) on several extensively used and publicly available datasets. These datasets include the CMU house sequence (Cho et al. 2010; Zhou and De la Torre 2015), the car and motorbike dataset (Zhou and De la Torre 2015; Leordeanu et al. 2012), and the Chinese character dataset (Liu and Qiao 2014; Zhang et al. 2016). The experiments of this part are performed on a desktop with 3.4 GHz Intel Core i5-7500 CPU, 8GB memory.

The CMU house sequence consists of 111 images of a toy house captured from different viewpoints. Each image has 30 manually marked landmark points with known correspondences. We match all images spaced by $5,10, \ldots, 110$ frames and compute the average performance per separation gap. The large gaps indicate more challenging scenes due to the increasing perspective changes. We build the graph using Delaunay triangulation and construct the affinity matrix simply by the edge distance as in Zhou and De la Torre (2015), except for TM, which has high order. Different from the original equal-size 30-node to 30-node matching, we remove some nodes and conduct unequal-size matching experiments with the corresponding settings of 25 versus 30 and 20 versus
30 on this dataset to test the robustness of these algorithms, as presented in Fig. 8. The figure shows that in the equalsize matching, most GM methods can achieve near-optimal performance, except for the spectral relaxed baselines. For unequal-size matching, the performance gap has emerged. In summary, FGM achieves the best performance with the highest time cost, and RRWM is the most balanced algorithm, which is only inferior to FGM in accuracy but is much more efficient.

The car and motorbike dataset consists of 30 pairs of car images and 20 pairs of motorbike images obtained from the PASCAL challenges (Everingham et al. 2010). Each pair contains 30-60 ground-truth correspondences. We consider the most general graph wherein the edge is directed and the edge feature is asymmetrical. Similarly, the graph is built with Delaunay triangulation, and the affinity matrix is constructed as in Zhou and De la Torre (2015) except for TM. To test the robustness to outliers, $2 \sim 20$ outliers are randomly selected from the background. As shown in Fig. 9, the path following algorithms, i.e., GNCCP and FGM, outperform all other methods, except for TM with the highest time cost. The RRWM remains competitive with high accuracy and low runtime. The higher-order TM has achieved remarkable performance in this experiment with consistent optimal performance. Moreover, its runtime is reasonable due to the adopted random sampling strategy used for constructing the three-order affinity matrix. The direct comparison of pairwise and higher-order graph matching methods can be unfair, but 

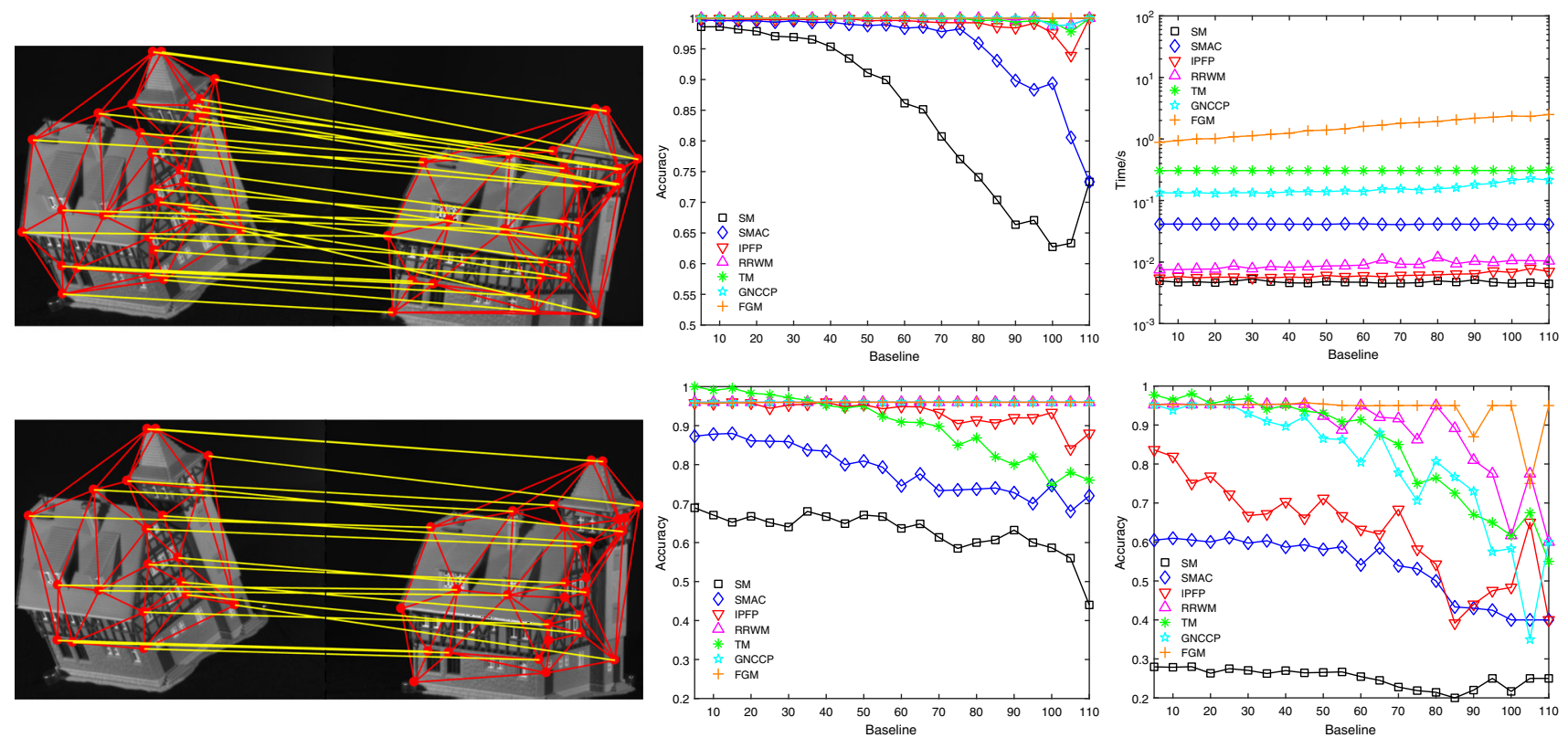

Fig. 8 Quantitative evaluation on the CMU house dataset. Top row (from left to right): illustration of equal-size matching with groundtruth correspondence, 30 versus 30 matching results and its runtime statistics. Bottom row (from left two right): example of unequal-size matching with ground-truth correspondence, 25 versus 30 matching results and 20 versus 30 matching results
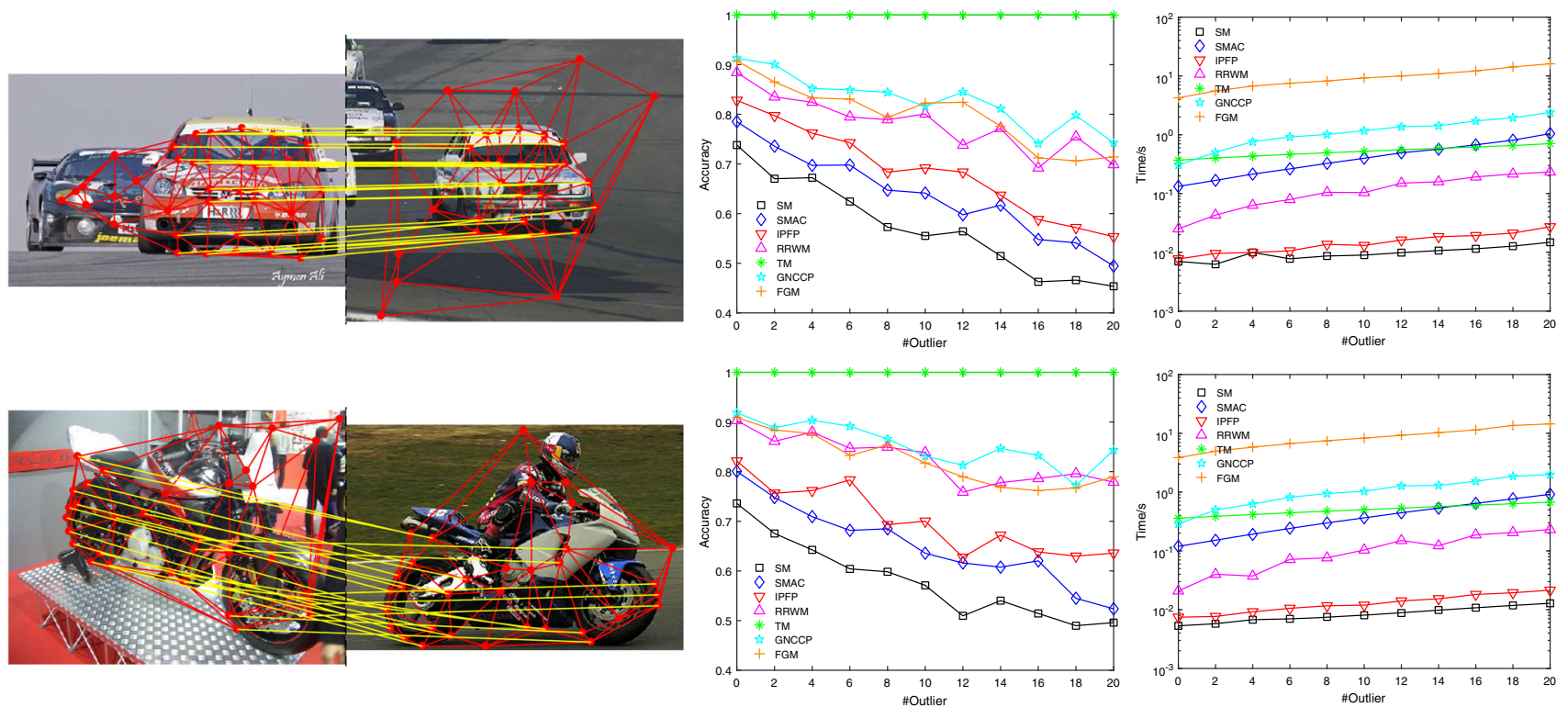

Fig. 9 Quantitative evaluation on car and motorbike dataset. Top row (from left to right): example of equal-size matching with ground-truth correspondence, car image matching results and the runtime statistics.
Bottom row (from left two right): example of unequal-size matching with ground-truth correspondence, motorbike matching results and the runtime statistics 


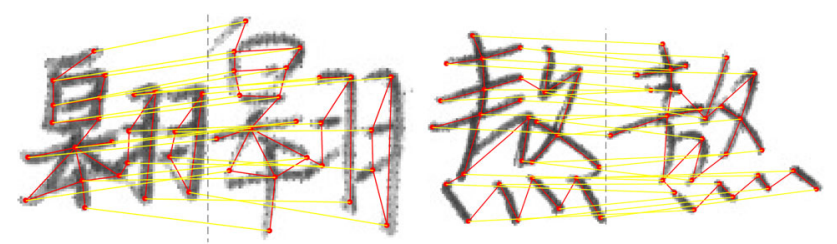

Fig. 10 Examples of the Chinese character dataset

the results still exhibit the efficacy of utilizing higher-order information in GM.

The Chinese character dataset has four hand-written Chinese characters with marked features wherein each character has 10 samples. We create matching instances between all pairs of samples for each character, i.e. 45 instances each. The average performance is summarized in Table 2 and the example is shown in Fig. 10. The scene is relatively challenging, and we use simple edge distances to construct affinity matrix, resulting in the relatively low accuracy for all methods. However, the superior performers are still evident. FGM and TM perform similarly, but TM is more efficient.

\subsection{Results on Pose Estimation}

The camera pose estimation aims to determine the position and orientation of the camera with respect to the object or scene, which is a significant step in 3-D computer vision tasks, such as SfM, SLAM, and visual localization for selfdriving cars and augmented reality. Here, the camera pose estimation of traditional approaches estimates the pose from a set of 2-D versus 3-D matches between pixels in a query image and 3-D points in a scene model. However, the 3-D model is typically obtained via SfM, thus leading to potentially inaccurate pose estimates. To address this problem, one alternative is to perform a set of 2-D versus 2-D correspondences between two or more images of the same scene.

To estimate the camera pose, the putative sparse feature correspondences must also be constructed with off-the-shelf feature matcher, such as SIFT. Moreover, the most classical pipeline is the combination of SIFT and RANSAC. The geometric model can be estimated and converted into the relative camera pose, i.e., rotation matrix and translation matrix. Many advanced handcrafted methods and trainable ones are considered as good options for their superior performance. Here, we integrate some typical mismatch removal methods between SIFT and RANSAC, while some learning-based methods can intrinsically output the transform matrix from their networks, which can be directly used for this task. In addition, two different datasets, including indoor and outdoor scenes, are used in this experiment. The performance is characterized by the mean average precision (mAP), as depicted in Table 3. The experiments of this part are performed on a server with $2.00 \mathrm{GHz}$ Intel Xeon CPU, 128 GB memory.
In the following, we briefly introduce the datasets and evaluation metrics to be used and provide quantitative comparisons and analyses.

Outdoor scenes. We adopt the Yahoo's YFCC100M dataset (Thomee et al. 2016), with 100 million publicly accessible tourist photos from the Internet and subsequently curate into 72 image sequences for SfM. From this dataset, 68 sequences are selected as valid raw data. Next, we use the Visual SfM (Heinly et al. 2015) to recover the camera poses and generate the ground-truth. This dataset is divided into disjoint subsets for training (60\%), validation (20\%), and test (20\%). For fairness, all learning-based methods are re-trained on the same training set.

Indoor scenes. We adopt the SUN3D dataset (Xiao et al. 2013), which is an RGBD video dataset with camera poses computed by generalized bundle adjustment. Specifically, all samples in this dataset are subsampled from videos of every 10 frames of feature office-like scenes. This dataset is extremely challenging for sparse correspondence methods due to the few distinctive features, heavy repetitive elements, and substantial self-occlusions. Zhang et al. (2019b) reported that some sequences in this dataset do not provide camera poses. Thus, these sequences are dropped and 239 sequences are finally obtained as valid data. Similar to the data of outdoor scenes, the SUN3D dataset is split into disjoint subsets for training (60\%), validation (20\%), and testing (20\%).

Evaluation Metrics. Once potential inliers are obtained, it is possible to efficiently estimate the rotation and translation vectors by RANSAC. The performance can be evaluated using the angular difference between the estimated and ground-truth vectors; i.e., the closest arc distance in degrees as the error metric. First, a curve should be generated by classifying whether each pose as accurate or not. The precision should be computed with respect to the given angle threshold from $0^{\circ}$ to $180^{\circ}$, and a normalized cumulative curve should be built. Second, the area under curve (AUC) is computed up to a maximum threshold of $5^{\circ}, 10^{\circ}$, or $20^{\circ}$. Since the curve itself can measure precision, its AUC can be regarded as the metric of mAP.

Several traditional mismatch removal methods, i.e., GMS (Bian et al. 2017), ICF (Li and Hu 2010), LPM (Ma et al. 2019d), SM (Leordeanu and Hebert 2005) and VFC (Ma et al. 2014) are used for evaluation of the pose estimation task, in addition to two deep-learning-based methods, i.e., LFGC (Moo Yi et al. 2018) and OAN (Zhang et al. 2019b). For these methods, pose estimation results are obtained by a subsequent RANSAC procedure. In addition, plain RANSAC (Fischler and Bolles 1981) is also included for comparison. As shown in Table 3, on the adopted dataset, the performances of traditional methods are very limited due to the dominant outliers. In contrast, the deep-learning-based methods seem to significantly outperform the traditional methods, resilient to the high outlier ratio. 


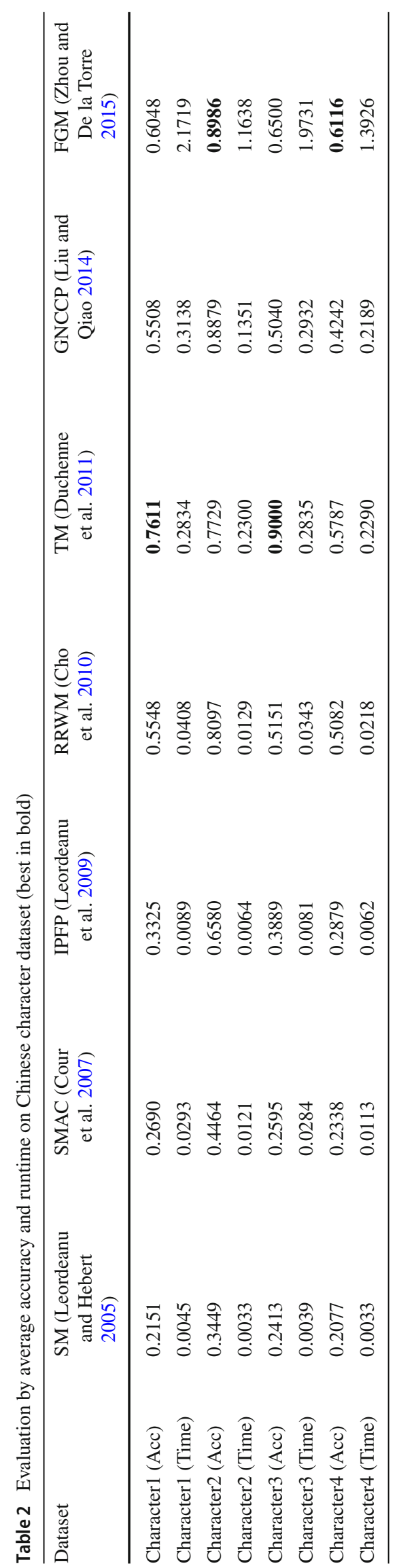




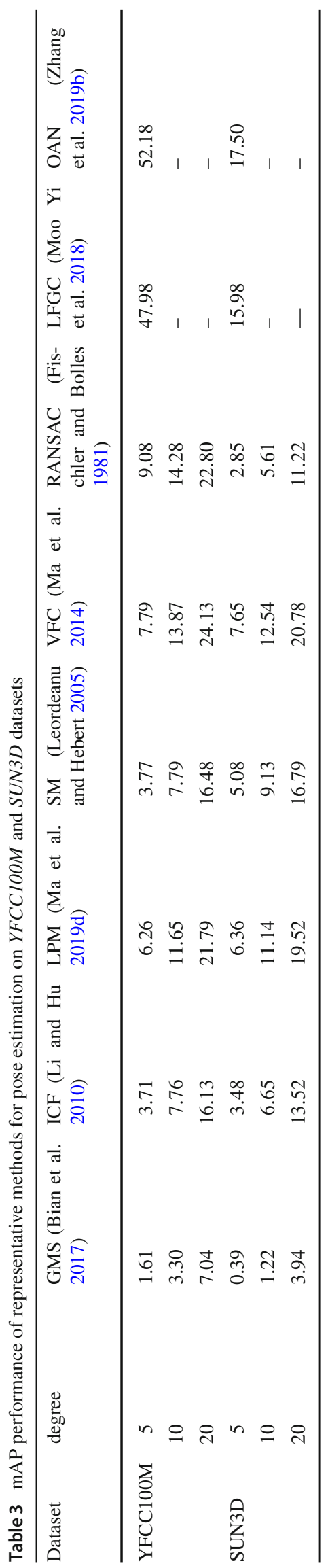




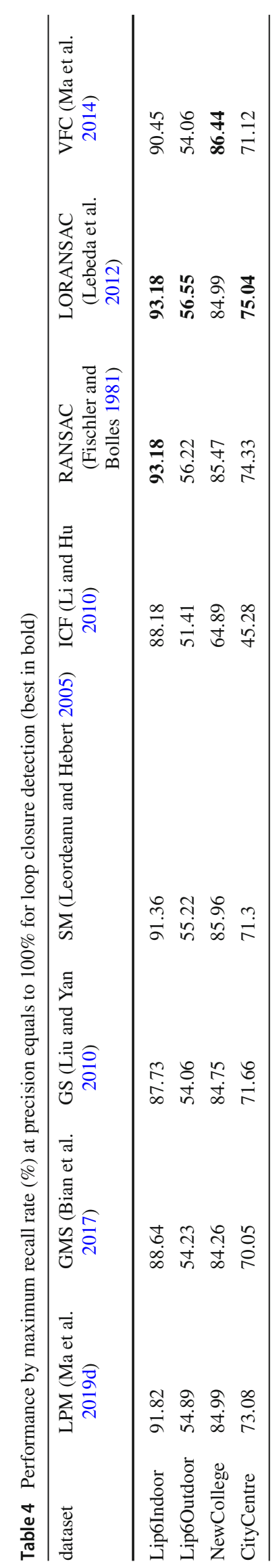




\subsection{Results on Loop-Closure Detection}

Appearance-based loop-closure detection is a fundamental component in visual SLAM. The essence involves recognizing previously visited areas of the environment. This task is crucial in reducing the drift of the estimated trajectory caused by the accumulative error and contributes to global consistent mapping.

Appearance-based loop-closure detection only uses image similarity to identify previously visited places. This category commonly starts with the construction of a set of putative correspondences by a feature operator, such as SIFT, between the current image and each previously visited image. Then, the closed loop is determined on the basis of the number of accurate matches using mismatch removal methods. This solution is simple but relatively effective.

Moreover, the computational requirement in directly realizing feature matching between the current image and each previously visited image would be largely increased. To ensure the real-time performance of loop-closure detection, we use a two-step approach. In the first step, loop-closure candidates are selected by the BoW method with presupposed score threshold, which is fast and easy to implement. However, the BoW method only considers whether or not a feature exists and neglects the spatial arrangement of the features, thereby leading to perceptual aliasing problem. Thus, in the second step, a robust feature matching algorithm is required to determine whether a loop-closure candidate is a true loop-closure event.

To evaluate the effectiveness and compare the performance of the loop-closure detection methods based on feature matching, we conduct extensive experiments on four different datasets, including NewCollege, CityCentre, Lip6Indoor, and Lip6Outdoor. The performance is characterized by the maximum recall that can be achieved at $100 \%$ precision, as shown in Table 4. The experiments are performed on a desktop with $2.6 \mathrm{GHz}$ Intel Core CPU, $16 G B$ memory.

The NewCollege and CityCentre datasets are obtained from the work of Cummins and Newman Cummins and Newman (2008). The NewCollege dataset contains 1, 073 images with size of $640 \times 480$, and the CityCentre dataset contains 1, 237 images with size of $640 \times 480$. The images were recorded by means of the vision system of a wheeled robotic platform while traversing $2.2 \mathrm{~km}$ through a college's campus grounds and adjoining parks with buildings, roads, gardens, cars, and people. The environment is outdoor and dynamic.

The Lip6Indoor and Lip6Outdoor datasets are obtained from Angeli et al. (2008). The Lip6Indoor dataset has 388 images with size of $240 \times 192$; it is an indoor image sequence with strong perceptual aliasing problem. While the Lip6Outdoor dataset has 1,063 images with size of $240 \times 192$; it is a long outdoor image sequence of a street with many buildings, cars, and people. Both image sequences are grabbed with a single-monocular handheld camera. In addition, a binary matrix is defined as the ground truth for each dataset, whose rows and columns correspond to images at different time indices. Each element in this binary matrix denotes the presence (set to 1) or absence (set to 0) of a loop-closure event between the corresponding frame pair.

To generate consistent maps, the loop-closure detection module should obtain true positive detections to provide information for the back-end optimization, thereby reducing the drift of the estimated trajectory caused by accumulative error. However, the loop-closure detection result must also include no false positive detections as this can affect the performance of a full SLAM system and result in a completely inaccurate map result. In summary, the loop closure mechanisms should work at $100 \%$ precision while maintaining high recall rate. In such cases, the evaluation of loop-closure detection algorithm is performed in terms of precision-recall metrics. Here, precision is the ratio of the number of true positive loop-closure detections to the number of total positive loop-closure detections identified by the system, and recall is the ratio between the true positive loop closure detections and the total actual loop-closure events defined by the ground truth of dataset. Combining the analysis and the curve, we focus on the maximum recall that can be achieved at $100 \%$ precision, indicating that the loop-closure detection result includes no false positive detection and avoids the influence in a full SLAM system.

Some of the representative mismatch removal methods are adopted for comparison in our experiment. The quantitative comparisons, with respect to maximum recall rate at precision of $100 \%$ on different datasets, are presented in Table 4. From the results, we can see that the methods that pursue relaxed geometric constraints, i.e., LPM (Ma et al. 2019d), GMS (Bian et al. 2017), GS (Liu and Yan 2010), SM (Leordeanu and Hebert 2005), ICF ( $\mathrm{Li}$ and $\mathrm{Hu}$ 2010) and VFC (Ma et al. 2014), are less favored in this task. In comparison, the resampling methods that exploit parametric models of the correspondences, i.e., RANSAC (Fischler and Bolles 1981) and LORANSAC (Lebeda et al. 2012), can give better results for loop-closure detection.

\section{Conclusions and Future Trends}

Image matching has played a significant role in various visual applications and has attracted considerable attention. Researchers have also achieved significant progress in this field in the past few decades. Therefore, we provide a comprehensive review of the existing image matching methods-from handcrafted to trainable ones-in order to provide better reference and understanding for the researchers in this community. 
Image matching can be briefly classified into area- and feature-based matching. Area-based methods are used to achieve dense matching without detecting any salient feature points from the images. They are more welcomed in high overlapping image matching (such as medical image registration) and narrow-baseline stereo (such as binocular stereo matching). The deep learning-based techniques have drawn increasing attention for such a pipeline. Therefore, we provide a brief review of these types of methods in Sect. 4 and focus more on the learning-based methods.

The feature-based image matching can effectively address the limitations in large viewpoint, wide baseline, and serious non-rigid image matching problems. It can be used in a pipeline of salient feature detection, discriminative description, and reliable matching, often including transformation model estimation. Following this procedure, feature detection can extract the distinctive structure from the image. Meanwhile, feature description may be regarded as an image representation method, which is widely used for image coding and similarity measurement. The matching step can be extended into different types of matching forms, such as graph matching, point set registration, descriptor matching and mismatch removal, as well as the matching task in 3-D cases. These are more flexible and applicable than area-based methods, thereby receiving considerable attention in image matching area. Therefore, we review them with the core idea that they are used from traditional techniques to classical learning and deep learning. Moreover, to provide a comprehensive understanding of the significance in image matching, we introduce several applications related to image matching. We also provide comprehensive and objective comparisons and analyses of these classical and deep learning-based techniques through extensive experiments on representative datasets.

Despite the considerable development in both theory and performance, image matching remains an open problem with challenges for further efforts.

- The two-stage strategy for feature matching, which has been widely adopted in the literature, performs mismatch removal on only a small set of potential correspondences with sufficiently similar descriptors. However, this may lead to restricted performance in recall, which can be problematic for some scenarios.

- In a different scenario, correspondences are sought not between projections of physically the same points in different images, but between semantic analogs across different instances within a category. This requires new paradigms for feature matching in feature description and mismatch removal.

- Joint matching of multiple images has been proven to drastically boost the matching performance of pairwise matching and has attracted considerable attention in recent years. However, the complexity is still the main concern of the problem. Thus, practical and efficient algorithms are required.

- In recent years, deep learning schemes have rapidly evolved and shown tremendous improvements in many research fields related to computer vision. However, in the literature of feature matching, most works have applied deep learning techniques to feature detection and description. Thus, the potential capacity for accurate feature matching can be further explored in the future.

- Image matching among multi-modal images is still an unsolved problem. In the future, deep learning techniques can be used for better feature detection and description performance.

- Feature matching is a fundamental task in computer vision. However, its application has not been sufficiently explored. Thus, one promising research direction is to customize modern feature matching techniques to satisfy different requirements of practical vision tasks, e.g., SfM and SLAM.

Acknowledgements This work was partly supported by the National Natural Science Foundation of China under Grant Nos. 61773295 and 61972250, Natural Science Foundation of Hubei Province under Grant No. 2019CFA037, and National Key Research and Development Program of China under Grant No. 2018AAA0100704.

\section{Compliance with ethical standards}

Conflict of Interest The authors declare no conflict of interest.

Open Access This article is licensed under a Creative Commons Attribution 4.0 International License, which permits use, sharing, adaptation, distribution and reproduction in any medium or format, as long as you give appropriate credit to the original author(s) and the source, provide a link to the Creative Commons licence, and indicate if changes were made. The images or other third party material in this article are included in the article's Creative Commons licence, unless indicated otherwise in a credit line to the material. If material is not included in the article's Creative Commons licence and your intended use is not permitted by statutory regulation or exceeds the permitted use, you will need to obtain permission directly from the copyright holder. To view a copy of this licence, visit http://creativecomm ons.org/licenses/by/4.0/.

\section{References}

Aanæs, H., Dahl, A. L., \& Pedersen, K. S. (2012). Interesting interest points. International Journal of Computer Vision, 97(1), 18-35.

Aanæs, H., Jensen, R. R., Vogiatzis, G., Tola, E., \& Dahl, A. B. (2016). Large-scale data for multiple-view stereopsis. International Journal of Computer Vision, 120(2), 153-168.

Abdel-Hakim, A. E., \& Farag, A. A. (2006). Csift: A sift descriptor with color invariant characteristics. In Proceedings of the IEEE conference on computer vision and pattern recognition, pp. 19781983. 
Adamczewski, K., Suh, Y., \& Mu Lee, K. (2015). Discrete tabu search for graph matching. In Proceedings of the IEEE international conference on computer vision, pp. 109-117.

Adams, W. P., \& Johnson, T. A. (1994). Improved linear programmingbased lower bounds for the quadratic assignment problem. DIMACS Series in Discrete Mathematics and Theoretical Computer Science, 16, 43-77.

Aflalo, Y., Dubrovina, A., \& Kimmel, R. (2016). Spectral generalized multi-dimensional scaling. International Journal of Computer Vision, 118(3), 380-392.

Agrawal, M., Konolige, K., \& Blas, M. R. (2008). Censure: Center surround extremas for realtime feature detection and matching. In Proceedings of the European conference on computer vision, pp. $102-115$.

Alahi, A., Ortiz, R., \& Vandergheynst, P. (2012). Freak: Fast retina keypoint. In Proceedings of the IEEE conference on computer vision and pattern recognition, pp. 510-517.

Alcantarilla, P. F., Bartoli, A., \& Davison, A. J. (2012) Kaze features. In Proceedings of the European conference on computer vision, pp. 214-227.

Alcantarilla, P. F., \& Solutions, T. (2011). Fast explicit diffusion for accelerated features in nonlinear scale spaces. IEEE Transactions on Pattern Analysis and Machine Intelligence, 34(7), 1281-1298.

Aldana-Iuit, J., Mishkin, D., Chum, O., \& Matas, J. (2016). In the saddle: Chasing fast and repeatable features. In Proceedings of the international conference on pattern recognition, pp. 675-680.

Almohamad, H., \& Duffuaa, S. O. (1993). A linear programming approach for the weighted graph matching problem. IEEE Transactions on Pattern Analysis and Machine Intelligence, 15(5), $522-525$.

Amberg, B., Romdhani, S., \& Vetter, T. (2007). Optimal step nonrigid ICP algorithms for surface registration. In Proceedings of the IEEE conference on computer vision and pattern recognition, pp. 1-8.

Angeli, A., Filliat, D., Doncieux, S., \& Meyer, J. A. (2008). A fast and incremental method for loop-closure detection using bags of visual words. In: IEEE transactions on robotics, pp. 1027-1037.

Arandjelović, R., \& Zisserman, A. (2012). Three things everyone should know to improve object retrieval. In Proceedings of the IEEE conference on computer vision and pattern recognition, pp. 29112918.

Arar, M., Ginger, Y., Danon, D., Bermano, A. H., \& Cohen-Or, D. (2020). Unsupervised multi-modal image registration via geometry preserving image-to-image translation. In Proceedings of the IEEE/CVF conference on computer vision and pattern recognition, pp. 13,410-13,419.

Aubry, M., Schlickewei, U., \& Cremers, D. (2011). The wave kernel signature: A quantum mechanical approach to shape analysis. In Proceedings of the IEEE international conference on computer vision workshops, pp. 1626-1633.

Avrithis, Y., \& Tolias, G. (2014). Hough pyramid matching: Speeded-up geometry re-ranking for large scale image retrieval. International Journal of Computer Vision, 107(1), 1-19.

Awrangjeb, M., \& Lu, G. (2008). Robust image corner detection based on the chord-to-point distance accumulation technique. IEEE Transactions on Multimedia, 10(6), 1059-1072.

Awrangjeb, M., Lu, G., \& Fraser, C. S. (2012). Performance comparisons of contour-based corner detectors. IEEE Transactions on Image Processing, 21(9), 4167-4179.

Babai, L. (2018). Groups, graphs, algorithms: The graph isomorphism problem. In Proceedings of the international congress of mathematicians, pp. 3319-3336.

Balntas, V., Johns, E., Tang, L., \& Mikolajczyk, K. (2016a). Pn-net: Conjoined triple deep network for learning local image descriptors. arXiv preprint arXiv: 1601.05030

Balntas, V., Lenc, K., Vedaldi, A., \& Mikolajczyk, K. (2017). Hpatches: A benchmark and evaluation of handcrafted and learned local descriptors. In Proceedings of the IEEE conference on computer vision and pattern recognition, pp. 5173-5182.

Balntas, V., Riba, E., Ponsa, D., \& Mikolajczyk, K. (2016b). Learning local feature descriptors with triplets and shallow convolutional neural networks. In Proceedings of the British machine vision conference, pp. 1-11.

Barath, D., \& Matas, J. (2018). Graph-cut ransac. In Proceedings of the IEEE conference on computer vision and pattern recognition, $\mathrm{pp}$. 6733-6741.

Barath, D., Ivashechkin, M., \& Matas, J. (2019a). Progressive napsac: Sampling from gradually growing neighborhoods. arXiv preprint arXiv:1906.02295.

Barath, D., Matas, J., \& Noskova, J. (2019b). Magsac: Marginalizing sample consensus. In Proceedings of the IEEE conference on computer vision and pattern recognition, pp. 10,197-10,205.

Barath, D., Noskova, J., Ivashechkin, M., \& Matas, J. (2020). Magsac++, a fast, reliable and accurate robust estimator. In Proceedings of the IEEE/CVF conference on computer vision and pattern recognition, pp. 1304-1312.

Barroso-Laguna, A., Riba, E., Ponsa, D., \& Mikolajczyk, K. (2019). Key.net: Keypoint detection by handcrafted and learned CNN filters. In Proceedings of the IEEE international conference on computer vision, pp. 5836-5844.

Bay, H., Tuytelaars, T., \& Van Gool, L. (2006). Surf: Speeded up robust features. In Proceedings of the European conference on computer vision, pp. 404-417.

Bay, H., Ess, A., Tuytelaars, T., \& Van Gool, L. (2008). Speeded-up robust features (surf). Computer Vision and Image Understanding, 110(3), 346-359.

Bazin, J.C., Seo, Y., \& Pollefeys, M. (2012). Globally optimal consensus set maximization through rotation search. In Proceedings of the Asian conference on computer vision, pp. 539-551.

Bellavia, F., \& Colombo, C. (2020). Is there anything new to say about sift matching? International Journal of Computer Vision, 128(3), 1847-1866.

Belongie, S., Malik, J., \& Puzicha, J. (2001). Shape context: A new descriptor for shape matching and object recognition. In Advances in neural information processing systems, pp. 831-837.

Belongie, S., Malik, J., \& Puzicha, J. (2002). Shape matching and object recognition using shape contexts. IEEE Transactions on Pattern Analysis and Machine Intelligence, 4, 509-522.

Bernard, F., Theobalt, C., \& Moeller, M. (2018). Ds*: Tighter liftingfree convex relaxations for quadratic matching problems. In Proceedings of the IEEE conference on computer vision and pattern recognition, pp. 4310-4319.

Bernard, F., Thunberg, J., Swoboda, P., \& Theobalt, C. (2019). Hippi: Higher-order projected power iterations for scalable multimatching. In Proceedings of the IEEE international conference on computer vision, pp. 10,284-10,293.

Besl, P. J., \& McKay, N. D. (1992). Method for registration of 3-d shapes. In Sensor fusion IV: Control paradigms and data structures, Vol. 1611, pp. 586-607.

Bhattacharjee, D., \& Roy, H. (2019). Pattern of local gravitational force (plgf): A novel local image descriptor. In IEEE transactions on pattern analysis and machine intelligence.

Bhowmik, A., Gumhold, S., Rother, C., \& Brachmann, E. (2020). Reinforced feature points: Optimizing feature detection and description for a high-level task. In Proceedings of the IEEE/CVF conference on computer vision and pattern recognition, pp. 4948-4957.

Bian, J., Lin, W. Y., Matsushita, Y., Yeung, S. K., Nguyen, T. D., \& Cheng, M. M. (2017). Gms: Grid-based motion statistics for fast, ultra-robust feature correspondence. In Proceedings of the IEEE conference on computer vision and pattern recognition, pp. 41814190.

Biasotti, S., Cerri, A., Bronstein, A., \& Bronstein, M. (2016). Recent trends, applications, and perspectives in $3 \mathrm{~d}$ shape similarity assess- 
ment. In Computer graphics forum, Vol. 35, Wiley Online Library, pp. 87-119.

Blais, G., \& Levine, M. D. (1995). Registering multiview range data to create $3 \mathrm{~d}$ computer objects. IEEE Transactions on Pattern Analysis and Machine Intelligence, 17(8), 820-824.

Bonny, M. Z., \& Uddin, M. S. (2016). Feature-based image stitching algorithms. In Proceedings of the international workshop on computational intelligence, pp. 198-203.

Boscaini, D., Masci, J., Melzi, S., Bronstein, M. M., Castellani, U., \& Vandergheynst, P. (2015). Learning class-specific descriptors for deformable shapes using localized spectral convolutional networks. In Computer graphics forum, Vol. 34, Wiley Online Library, pp. 13-23.

Boscaini, D., Masci, J., Rodolà, E., \& Bronstein, M. (2016). Learning shape correspondence with anisotropic convolutional neural networks. In Advances in neural information processing systems, pp. 3189-3197.

Brachmann, E., \& Rother, C. (2019). Neural-guided RANSAC: Learning where to sample model hypotheses. In Proceedings of the IEEE international conference on computer vision, pp. 4322-4331.

Brachmann, E., Krull, A., Nowozin, S., Shotton, J., Michel, F., Gumhold, S., \& Rother, C. (2017). Dsac-differentiable RANSAC for camera localization. In Proceedings of the IEEE conference on computer vision and pattern recognition, pp. 6684-6692.

Bronstein, M. M., \& Kokkinos, I. (2010). Scale-invariant heat kernel signatures for non-rigid shape recognition. In Proceedings of the IEEE conference on computer vision and pattern recognition, $\mathrm{pp}$. $1704-1711$.

Bronstein, A. M., Bronstein, M. M., \& Kimmel, R. (2006). Generalized multidimensional scaling: a framework for isometry-invariant partial surface matching. Proceedings of the National Academy of Sciences, 103(5), 1168-1172.

Brown, M., Hua, G., \& Winder, S. (2010). Discriminative learning of local image descriptors. IEEE Transactions on Pattern Analysis and Machine Intelligence, 33(1), 43-57.

Brown, M., \& Lowe, D. G. (2007). Automatic panoramic image stitching using invariant features. International Journal of Computer Vision, 74(1), 59-73.

Caelli, T., \& Kosinov, S. (2004). An eigenspace projection clustering method for inexact graph matching. IEEE Transactions on Pattern Analysis and Machine Intelligence, 26(4), 515-519.

Caetano, T. S., McAuley, J. J., Cheng, L., Le, Q. V., \& Smola, A. J. (2009). Learning graph matching. IEEE Transactions on Pattern Analysis and Machine Intelligence, 31(6), 1048-1058.

Cai, H., Mikolajczyk, K., \& Matas, J. (2010). Learning linear discriminant projections for dimensionality reduction of image descriptors. IEEE Transactions on Pattern Analysis and Machine Intelligence, 33(2), 338-352.

Calonder, M., Lepetit, V., Strecha, C., \& Fua, P. (2010). Brief: Binary robust independent elementary features. In Proceedings of the European conference on computer vision, pp. 778-792.

Campbell, D., \& Petersson, L. (2015). An adaptive data representation for robust point-set registration and merging. In Proceedings of the IEEE international conference on computer vision, pp. 4292 4300.

Campbell, D., \& Petersson, L. (2016). Gogma: Globally-optimal gaussian mixture alignment. In Proceedings of the IEEE conference on computer vision and pattern recognition, pp. 5685-5694.

Canny, J. (1987). A computational approach to edge detection. In Readings in computer vision, Elsevier, pp. 184-203.

Cao, S. Y., Shen, H. L., Chen, S. J., \& Li, C. (2020). Boosting structure consistency for multispectral and multimodal image registration. IEEE Transactions on Image Processing, 29, 5147-5162.

Castellani, U., Cristani, M., Fantoni, S., \& Murino, V. (2008). Sparse points matching by combining $3 \mathrm{~d}$ mesh saliency with statistical descriptors. In Computer graphics forum, Vol. 27, Wiley Online Library, pp. 643-652.

Chang, J. R., \& Chen, Y. S. (2018). Pyramid stereo matching network. In Proceedings of the IEEE conference on computer vision and pattern recognition, pp. 5410-5418.

Chang, W., \& Zwicker, M. (2009). Range scan registration using reduced deformable models. In Computer graphics forum, Vol. 28, Wiley Online Library, pp. 447-456.

Chang, M. C., \& Kimia, B. B. (2011). Measuring 3d shape similarity by graph-based matching of the medial scaffolds. Computer Vision and Image Understanding, 115(5), 707-720.

Chen, Q., \& Koltun, V. (2015). Robust nonrigid registration by convex optimization. In Proceedings of the IEEE international conference on computer vision, pp. 2039-2047.

Chen, Y., Guibas, L., \& Huang, Q. (2014). Near-optimal joint object matching via convex relaxation. In Proceedings of the international conference on machine learning, pp. 100-108.

Chen, Y. C., Huang, P. H., Yu, L. Y., Huang, J. B., Yang, M. H., \& Lin, Y. Y. (2018). Deep semantic matching with foreground detection and cycle-consistency. In Proceedings of the Asian conference on computer vision, pp. 347-362.

Chen, J., Kellokumpu, V., Zhao, G., \& Pietikäinen, M. (2013). Rlbp: Robust local binary pattern. In Proceedings of the British machine vision conference.

Chen, J., Wang, L., Li, X., \& Fang, Y. (2019). Arbicon-net: Arbitrary continuous geometric transformation networks for image registration. In Advances in neural information processing systems, pp. 3410-3420.

Chen, H. M., Arora, M. K., \& Varshney, P. K. (2003a). Mutual information-based image registration for remote sensing data. International Journal of Remote Sensing, 24(18), 3701-3706.

Chen, H., \& Bhanu, B. (2007). 3d free-form object recognition in range images using local surface patches. Pattern Recognition Letters, 28(10), 1252-1262.

Chen, Q. S., Defrise, M., \& Deconinck, F. (1994). Symmetric phaseonly matched filtering of Fourier-Mellin transforms for image registration and recognition. IEEE Transactions on Pattern Analysis and Machine Intelligence, 16(12), 1156-1168.

Chen, C., Li, Y., Liu, W., \& Huang, J. (2015). Sirf: Simultaneous satellite image registration and fusion in a unified framework. IEEE Transactions on Image Processing, 24(11), 4213-4224.

Chen, J., Shan, S., He, C., Zhao, G., Pietikainen, M., Chen, X., et al. (2009). Wld: A robust local image descriptor. IEEE Transactions on Pattern Analysis and Machine Intelligence, 32(9), 1705-1720.

Chen, J., Tian, J., Lee, N., Zheng, J., Smith, R. T., \& Laine, A. F. (2010). A partial intensity invariant feature descriptor for multimodal retinal image registration. IEEE Transactions on Biomedical Engineering, 57(7), 1707-1718.

Chen, H. M., Varshney, P. K., \& Arora, M. K. (2003b). Performance of mutual information similarity measure for registration of multitemporal remote sensing images. IEEE Transactions on Geoscience and Remote Sensing, 41(11), 2445-2454.

Chertok, M., \& Keller, Y. (2010). Efficient high order matching. IEEE Transactions on Pattern Analysis and Machine Intelligence, 32(12), 2205-2215.

Chetverikov, D., Stepanov, D., \& Krsek, P. (2005). Robust Euclidean alignment of $3 \mathrm{~d}$ point sets: The trimmed iterative closest point algorithm. Image and Vision Computing, 23(3), 299-309.

Cho, M., \& Lee, K. M. (2012). Progressive graph matching: Making a move of graphs via probabilistic voting. In Proceedings of the IEEE conference on computer vision and pattern recognition, pp. 398-405.

Cho, M., Lee, J., \& Lee, K. M. (2010). Reweighted random walks for graph matching. In Proceedings of the European conference on computer vision, pp. 492-505. 
Chopra, S., Hadsell, R., LeCun, Y., et al. (2005). Learning a similarity metric discriminatively, with application to face verification. In Proceedings of the IEEE conference on computer vision and pattern recognition, pp. 539-546.

Choy, C. B., Gwak, J., Savarese, S., \& Chandraker, M. (2016). Universal correspondence network. In Advances in neural information processing systems, pp. 2414-2422.

Choy, C., Lee, J., Ranftl, R., Park, J., \& Koltun, V. (2020). Highdimensional convolutional networks for geometric pattern recognition. In Proceedings of the IEEE/CVF conference on computer vision and pattern recognition, pp. 11,227-11,236.

Chui, H., \& Rangarajan, A. (2003). A new point matching algorithm for non-rigid registration. Computer Vision and Image Understanding, 89(2-3), 114-141.

Chum, O., \& Matas, J. (2005). Matching with prosac-progressive sample consensus. In Proceedings of the IEEE conference on computer vision and pattern recognition, pp. 220-226.

Chum, O., Matas, J., \& Kittler, J. (2003). Locally optimized ransac. In Proceedings of the joint pattern recognition symposium, Springer, pp. 236-243.

Churchill, D., \& Vardy, A. (2013). An orientation invariant visual homing algorithm. Journal of Intelligent \& Robotic Systems, 71(1), 3-29.

Cook, D. J., \& Holder, L. B. (2006). Mining graph data. New York: Wiley.

Cour, T., Srinivasan, P., \& Shi, J. (2007). Balanced graph matching. In Advances in neural information processing systems, pp. 313-320.

Cummins, M., \& Newman, P. (2008). Fab-map: Probabilistic localization and mapping in the space of appearance. The International Journal of Robotics Research, 27(6), 647-665.

Dalal, N., \& Triggs, B. (2005). Histograms of oriented gradients for human detection. In Proceedings of the IEEE conference on computer vision and pattern recognition, pp. 886-893.

Danelljan, M., Meneghetti, G., Shahbaz Khan, F., \& Felsberg, M. (2016). A probabilistic framework for color-based point set registration. In Proceedings of the IEEE conference on computer vision and pattern recognition, pp. 1818-1826.

Datar, M., Immorlica, N., Indyk, P., \& Mirrokni, V. S. (2004). Localitysensitive hashing scheme based on p-stable distributions. In Proceedings of the twentieth annual symposium on computational geometry, pp. 253-262.

Davison, A. J., Reid, I. D., Molton, N. D., \& Stasse, O. (2007). Monoslam: Real-time single camera slam. IEEE Transactions on Pattern Analysis and Machine Intelligence, 6, 1052-1067.

Dawn, S., Saxena, V., \& Sharma, B. (2010). Remote sensing image registration techniques: A survey. In Proceedings of the international conference on image and signal processing, pp. 103-112.

de Vos, B. D., Berendsen, F. F., Viergever, M. A., Sokooti, H., Staring, M., \& Isgum, I. (2019). A deep learning framework for unsupervised affine and deformable image registration. Medical Image Analysis, 52, 128-143.

de Vos, B. D., Berendsen, F. F., Viergever, M. A., Staring, M., \& Isgum, I. (2017). End-to-end unsupervised deformable image registration with a convolutional neural network. In Deep learning in medical image analysis and multimodal learning for clinical decision support, Springer, pp. 204-212.

Deng, H., Birdal, T., \& Ilic, S. (2018). Ppfnet: Global context aware local features for robust $3 \mathrm{~d}$ point matching. In Proceedings of the IEEE conference on computer vision and pattern recognition, $\mathrm{pp}$. 195-205.

Deng, H., Zhang, W., Mortensen, E., Dietterich, T., \& Shapiro, L. (2007). Principal curvature-based region detector for object recognition. In Proceedings of the IEEE conference on computer vision and pattern recognition, pp. 1-8.

DeTone, D., Malisiewicz, T., \& Rabinovich, A. (2016). Deep image homography estimation. arXiv preprint arXiv:1606.03798.
DeTone, D., Malisiewicz, T., \& Rabinovich, A. (2018). Superpoint: Self-supervised interest point detection and description. In Proceedings of the IEEE conference on computer vision and pattern recognition workshops, pp. 224-236.

Dong, J., \& Soatto, S. (2015). Domain-size pooling in local descriptors: Dsp-sift. In Proceedings of the IEEE conference on computer vision and pattern recognition, pp. 5097-5106.

Dorai, C., \& Jain, A. K. (1997). Cosmos-a representation scheme for $3 \mathrm{~d}$ free-form objects. IEEE Transactions on Pattern Analysis and Machine Intelligence, 19(10), 1115-1130.

Dosovitskiy, A., Fischer, P., Ilg, E., Hausser, P., Hazirbas, C., Golkov, V., Van Der Smagt, P., Cremers, D., \& Brox, T. (2015). Flownet: Learning optical flow with convolutional networks. In Proceedings of the IEEE international conference on computer vision, $\mathrm{pp}$. 2758-2766.

Duan, Y., Lu, J., Wang, Z., Feng, J., \& Zhou, J. (2017). Learning deep binary descriptor with multi-quantization. In Proceedings of the IEEE conference on computer vision and pattern recognition, pp. 1183-1192.

Duchenne, O., Bach, F., Kweon, I. S., \& Ponce, J. (2011). A tensor-based algorithm for high-order graph matching. IEEE Transactions on Pattern Analysis and Machine Intelligence, 33(12), 2383-2395.

Du, Q., Fan, A., Ma, Y., Fan, F., Huang, J., \& Mei, X. (2018). Infrared and visible image registration based on scale-invariant piifd feature and locality preserving matching. IEEE Access, 6, 64107-64121.

Dusmanu, M., Rocco, I., Pajdla, T., Pollefeys, M., Sivic, J., Torii, A., \& Sattler, T. (2019). D2-net: A trainable cnn for joint description and detection of local features. In Proceedings of the IEEE conference on computer vision and pattern recognition, pp. 8092-8101.

Dym, N., Maron, H., \& Lipman, Y. (2017). Ds++: A flexible, scalable and provably tight relaxation for matching problems. arXiv preprint arXiv:1705.06148.

Egozi, A., Keller, Y., \& Guterman, H. (2012). A probabilistic approach to spectral graph matching. IEEE Transactions on Pattern Analysis and Machine Intelligence, 35(1), 18-27.

Elad, A., \& Kimmel, R. (2003). On bending invariant signatures for surfaces. IEEE Transactions on Pattern Analysis and Machine Intelligence, 25(10), 1285-1295.

Elbaz, G., Avraham, T., \& Fischer, A. (2017). 3d point cloud registration for localization using a deep neural network auto-encoder. In Proceedings of the IEEE conference on computer vision and pattern recognition, pp. 4631-4640.

Endres, F., Hess, J., Engelhard, N., Sturm, J., Cremers, D., \& Burgard, W. (2012). An evaluation of the rgb-d slam system. In Proceedings of the IEEE international conference on robotics and automation, pp. 1691-1696.

Erin Liong, V., Lu, J., Wang, G., Moulin, P., \& Zhou, J. (2015). Deep hashing for compact binary codes learning. In Proceedings of the IEEE conference on computer vision and pattern recognition, $\mathrm{pp}$. $2475-2483$.

Erlik Nowruzi, F., Laganiere, R., \& Japkowicz, N. (2017). Homography estimation from image pairs with hierarchical convolutional networks. In Proceedings of the IEEE international conference on computer vision, pp. 913-920.

Evangelidis, G. D., \& Horaud, R. (2018). Joint alignment of multiple point sets with batch and incremental expectation-maximization. IEEE Transactions on Pattern Analysis and Machine Intelligence, 40(6), 1397-1410.

Everingham, M., Van Gool, L., Williams, C. K., Winn, J., \& Zisserman, A. (2010). The pascal visual object classes (voc) challenge. International Journal of Computer Vision, 88(2), 303-338.

Fan, B., Kong, Q., Wang, X., Wanga, Z., Xiang, S., Pan, C., et al. (2019). A performance evaluation of local features for image-based $3 \mathrm{~d}$ reconstruction. IEEE Transactions on Image Processing, 28(10), 4774-4789. 
Fan, B., Wu, F., \& Hu, Z. (2011). Rotationally invariant descriptors using intensity order pooling. IEEE Transactions on Pattern Analysis and Machine Intelligence, 34(10), 2031-2045.

Ferrante, E., \& Paragios, N. (2017). Slice-to-volume medical image registration: A survey. Medical Image Analysis, 39, 101-123.

Ferraz, L., \& Binefa, X. (2012). A sparse curvature-based detector of affine invariant blobs. Computer Vision and Image Understanding, 116(4), 524-537.

Fey, M., Lenssen, J. E., Morris, C., Masci, J., \& Kriege, N. M. (2020). Deep graph matching consensus. In International conference on learning representations.

Fischler, M. A., \& Bolles, R. C. (1981). Random sample consensus: a paradigm for model fitting with applications to image analysis and automated cartography. Communications of the ACM, 24(6), 381-395.

Fitzgibbon, A. W. (2003). Robust registration of $2 d$ and $3 d$ point sets. Image and Vision Computing, 21(13-14), 1145-1153.

Flint, A., Dick, A., \& Van Den Hengel, A. (2007). Thrift: Local 3d structure recognition. In Proceedings of the biennial conference on digital image computing techniques and applications, pp. 182188

Fogel, F., Jenatton, R., Bach, F., \& d'Aspremont, A. (2013). Convex relaxations for permutation problems. In Advances in neural information processing systems, pp. 1016-1024.

Foroosh, H., Zerubia, J. B., \& Berthod, M. (2002). Extension of phase correlation to subpixel registration. IEEE Transactions on Image Processing, 11(3), 188-200.

Forssén, P. E. (2007). Maximally stable colour regions for recognition and matching. In Proceedings of the IEEE conference on computer vision and pattern recognition, pp. 1-8.

Fragoso, V., Sen, P., Rodriguez, S., \& Turk, M. (2013). Evsac: accelerating hypotheses generation by modeling matching scores with extreme value theory. In Proceedings of the IEEE international conference on computer vision, pp. 2472-2479.

Frome, A., Huber, D., Kolluri, R., Bülow, T., \& Malik, J. (2004). Recognizing objects in range data using regional point descriptors. In Proceedings of the European conference on computer vision, pp. 224-237.

Gao, W., \& Tedrake, R. (2019). Filterreg: Robust and efficient probabilistic point-set registration using Gaussian filter and twist parameterization. In Proceedings of the IEEE conference on computer vision and pattern recognition, pp. 11,095-11,104.

Gauglitz, S., Höllerer, T., \& Turk, M. (2011). Evaluation of interest point detectors and feature descriptors for visual tracking. International Journal of Computer Vision, 94(3), 335-360.

Gay-Bellile, V., Bartoli, A., \& Sayd, P. (2008). Direct estimation of nonrigid registrations with image-based self-occlusion reasoning. IEEE Transactions on Pattern Analysis and Machine Intelligence, 32(1), 87-104.

Gelfand, N., Mitra, N. J., Guibas, L. J., \& Pottmann, H. (2005). Robust global registration. In Symposium on geometry processing, Vol. 2, Vienna, Austria, p. 5.

Georgakis, G., Karanam, S., Wu, Z., Ernst, J., \& Kosecká, J. (2018). End-to-end learning of keypoint detector and descriptor for pose invariant $3 \mathrm{~d}$ matching. In Proceedings of the IEEE conference on computer vision and pattern recognition, pp. 1965-1973.

Ghosh, D., \& Kaabouch, N. (2016). A survey on image mosaicing techniques. Journal of Visual Communication and Image Representation, 34, 1-11.

Gil, A., Mozos, O. M., Ballesta, M., \& Reinoso, O. (2010). A comparative evaluation of interest point detectors and local descriptors for visual slam. Machine Vision and Applications, 21(6), 905-920.

Gionis, A., Indyk, P., Motwani, R., et al. (1999). Similarity search in high dimensions via hashing. In Proceedings of the international conference on very large databases, pp. 518-529.
Giraldo, L. G. S., Hasanbelliu, E., Rao, M., \& Principe, J. C. (2017). Group-wise point-set registration based on rényi's second order entropy. In Proceedings of the IEEE conference on computer vision and pattern recognition, pp. 2454-2462.

Glaunes, J., Trouvé, A., \& Younes, L. (2004). Diffeomorphic matching of distributions: A new approach for unlabelled point-sets and submanifolds matching. In Proceedings of the IEEE conference on computer vision and pattern recognition, pp. 712-718.

Gojcic, Z., Zhou, C., Wegner, J. D., Guibas, L. J., \& Birdal, T. (2020). Learning multiview $3 \mathrm{~d}$ point cloud registration. In Proceedings of the IEEE/CVF conference on computer vision and pattern recognition, pp. 1759-1769.

Gold, S., \& Rangarajan, A. (1996). A graduated assignment algorithm for graph matching. IEEE Transactions on Pattern Analysis and Machine Intelligence, 18(4), 377-388.

Gold, S., Rangarajan, A., Lu, C. P., Pappu, S., \& Mjolsness, E. (1998). New algorithms for $2 \mathrm{~d}$ and $3 \mathrm{~d}$ point matching: Pose estimation and correspondence. Pattern Recognition, 31(8), 1019-1031.

Golyanik, V., Aziz Ali, S., \& Stricker, D. (2016). Gravitational approach for point set registration. In Proceedings of the IEEE conference on computer vision and pattern recognition, pp. 5802-5810.

Gong, Y., Kumar, S., Rowley, H. A., \& Lazebnik, S. (2013). Learning binary codes for high-dimensional data using bilinear projections. In Proceedings of the IEEE conference on computer vision and pattern recognition, pp. 484-491.

Gong, Y., Lazebnik, S., Gordo, A., \& Perronnin, F. (2012). Iterative quantization: A procrustean approach to learning binary codes for large-scale image retrieval. IEEE Transactions on Pattern Analysis and Machine Intelligence, 35(12), 2916-2929.

Goodfellow, I., Pouget-Abadie, J., Mirza, M., Xu, B., Warde-Farley, D., Ozair, S., Courville, A., \& Bengio, Y. (2014). Generative adversarial nets. In Advances in neural information processing systems, pp. 2672-2680.

Granger, S., \& Pennec, X. (2002). Multi-scale em-icp: A fast and robust approach for surface registration. In Proceedings of the European conference on computer vision, pp. 418-432.

Guo, Y., Bennamoun, M., Sohel, F., Lu, M., Wan, J., \& Kwok, N. M. (2016). A comprehensive performance evaluation of 3d local feature descriptors. International Journal of Computer Vision, 116(1), 66-89.

Guo, Y., Sohel, F., Bennamoun, M., Lu, M., \& Wan, J. (2013). Rotational projection statistics for $3 \mathrm{~d}$ local surface description and object recognition. International Journal of Computer Vision, 105(1), 63-86.

Guo, Y., Sohel, F., Bennamoun, M., Wan, J., \& Lu, M. (2015). A novel local surface feature for $3 \mathrm{~d}$ object recognition under clutter and occlusion. Information Sciences, 293, 196-213.

Guo, Z., Zhang, L., \& Zhang, D. (2010). A completed modeling of local binary pattern operator for texture classification. IEEE Transactions on Image Processing, 19(6), 1657-1663.

Gupta, R., Patil, H., \& Mittal, A. (2010). Robust order-based methods for feature description. In Proceedings of the IEEE conference on computer vision and pattern recognition, pp. 334-341.

Han, X., Leung, T., Jia, Y., Sukthankar, R., \& Berg, A. C. (2015). Matchnet: Unifying feature and metric learning for patch-based matching. In Proceedings of the IEEE conference on computer vision and pattern recognition, pp. 3279-3286.

Han, K., Rezende, R. S., Ham, B., Wong, K. Y. K., Cho, M., Schmid, C., \& Ponce, J. (2017). Scnet: Learning semantic correspondence. In Proceedings of the IEEE international conference on computer vision, pp. 1831-1840.

Harris, C. G., Stephens, M., et al. (1988). A combined corner and edge detector. In Proceedings of the Alvey vision conference, pp. $147-$ 151. 
Hartmann, W., Havlena, M., \& Schindler, K. (2014). Predicting matchability. In Proceedings of the IEEE conference on computer vision and pattern recognition, pp. 9-16.

Haskins, G., Kruger, U., \& Yan, P. (2020). Deep learning in medical image registration: A survey. Machine Vision and Applications, $31(1), 8$.

Hayat, N., \& Imran, M. (2019). Ghost-free multi exposure image fusion technique using dense sift descriptor and guided filter. Journal of Visual Communication and Image Representation, 62, 295-308.

He, K., Lu, Y., \& Sclaroff, S. (2018). Local descriptors optimized for average precision. In Proceedings of the IEEE conference on computer vision and pattern recognition, pp. 596-605.

Heikkilä, M., Pietikäinen, M., \& Schmid, C. (2009). Description of interest regions with local binary patterns. Pattern Recognition, 42(3), 425-436.

Heinly, J., Dunn, E., \& Frahm, J. M. (2012). Comparative evaluation of binary features. In Proceedings of the European conference on computer vision, pp. 759-773.

Heinly, J., Schonberger, J. L., Dunn, E., \& Frahm, J. M. (2015). Reconstructing the world* in six days*(as captured by the yahoo 100 million image dataset). In Proceedings of the IEEE conference on computer vision and pattern recognition, pp. 3287-3295.

Hinterstoisser, S., Lepetit, V., Ilic, S., Holzer, S., Bradski, G., Konolige, K., \& Navab, N. (2012). Model based training, detection and pose estimation of texture-less $3 \mathrm{~d}$ objects in heavily cluttered scenes. InProceedings of the Asian conference on computer vision, pp. $548-562$.

Horaud, R., Forbes, F., Yguel, M., Dewaele, G., \& Zhang, J. (2011). Rigid and articulated point registration with expectation conditional maximization. IEEE Transactions on Pattern Analysis and Machine Intelligence, 33(3), 587-602.

Hu, N., Huang, Q., Thibert, B., \& Guibas, L. J. (2018). Distributable consistent multi-object matching. In Proceedings of the IEEE conference on computer vision and pattern recognition, pp. 24632471.

Huang, Q. X., \& Guibas, L. (2013). Consistent shape maps via semidefinite programming. In Computer graphics forum, Vol. 32, Wiley Online Library, pp. 177-186.

Huang, X., Cheng, X., Geng, Q., Cao, B., Zhou, D., Wang, P., Lin, Y., \& Yang, R. (2018). The apolloscape dataset for autonomous driving. In Proceedings of the IEEE conference on computer vision and pattern recognition workshops, pp. 954-960.

Huang, D., Shan, C., Ardabilian, M., Wang, Y., \& Chen, L. (2011). Local binary patterns and its application to facial image analysis: a survey. IEEE Transactions on Systems, Man, and Cybernetics, Part C (Applications and Reviews), 41(6), 765-781.

Iglesias, J. P., Olsson, C., \& Kahl, F. (2020). Global optimality for point set registration using semidefinite programming. In Proceedings of the IEEE/CVF conference on computer vision and pattern recognition, pp. 8287-8295.

Jaderberg, M., Simonyan, K., Zisserman, A., et al. (2015). Spatial transformer networks. In Advances in neural information processing systems, pp. 2017-2025.

Jégou, H., Douze, M., \& Schmid, C. (2010). Improving bag-of-features for large scale image search. International Journal of Computer Vision, 87(3), 316-336.

Jiang, B., Tang, J., Ding, C., \& Luo, B. (2017b). Binary constraint preserving graph matching. In Proceedings of the IEEE conference on computer vision and pattern recognition, pp. 4402-4409.

Jiang, B., Tang, J., Ding, C., Gong, Y., \& Luo, B. (2017a). Graph matching via multiplicative update algorithm. In Advances in neural information processing systems, pp. 3187-3195.

Jiang, Z., Wang, T., \& Yan, J. (2020b). Unifying offline and online multi-graph matching via finding shortest paths on supergraph. In IEEE transactions on pattern analysis and machine intelligence.
Jiang, X., Ma, J., Jiang, J., \& Guo, X. (2020a). Robust feature matching using spatial clustering with heavy outliers. IEEE Transactions on Image Processing, 29, 736-746.

Jiang, B., Zhao, H., Tang, J., \& Luo, B. (2014). A sparse nonnegative matrix factorization technique for graph matching problems. Pattern Recognition, 47(2), 736-747.

Jian, B., \& Vemuri, B. C. (2011). Robust point set registration using Gaussian mixture models. IEEE Transactions on Pattern Analysis and Machine Intelligence, 33(8), 1633-1645.

Jin, Y., Mishkin, D., Mishchuk, A., Matas, J., Fua, P., Yi, K. M., \& Trulls, E. (2020). Image matching across wide baselines: From paper to practice. arXiv preprint arXiv:2003.01587.

Johnson, K., Cole-Rhodes, A., Zavorin, I., \& Le Moigne, J. (2001). Mutual information as a similarity measure for remote sensing image registration. In Geo-spatial image and data exploitation II, pp. 51-61.

Johnson, A. E., \& Hebert, M. (1999). Using spin images for efficient object recognition in cluttered 3d scenes. IEEE Transactions on Pattern Analysis and Machine Intelligence, 21(5), 433-449.

Ke, Y., Sukthankar, R., et al. (2004). Pca-sift: A more distinctive representation for local image descriptors. In Proceedings of the IEEE conference on computer vision and pattern recognition, pp. 506513.

Kedem, D., Tyree, S., Sha, F., Lanckriet, G. R., \& Weinberger, K. Q. (2012). Non-linear metric learning. In Advances in neural information processing systems, pp. 2573-2581.

Kezurer, I., Kovalsky, S. Z., Basri, R., \& Lipman, Y. (2015). Tight relaxation of quadratic matching. In Computer graphics forum, Vol. 34, Wiley Online Library, pp. 115-128.

Khoury, M., Zhou, Q. Y., \& Koltun, V. (2017). Learning compact geometric features. In Proceedings of the IEEE international conference on computer vision, pp. 153-161.

Kim, S., Lin, S., JEON, S. R., Min, D., \& Sohn, K. (2018). Recurrent transformer networks for semantic correspondence. In Advances in neural information processing systems, pp. 6126-6136.

Kim, V.G., Lipman, Y., \& Funkhouser, T. (2011). Blended intrinsic maps. In ACM transactions on graphics, Vol. 30, ACM, p. 79.

Kim, V. G., Li, W., Mitra, N. J., DiVerdi, S., \& Funkhouser, T. A. (2012). Exploring collections of $3 \mathrm{~d}$ models using fuzzy correspondences. ACM Transactions on Graphics, 31(4), 54-1.

Kimmel, R., Zhang, C., Bronstein, A., \& Bronstein, M. (2011). Are mser features really interesting? IEEE Transactions on Pattern Analysis and Machine Intelligence, 33(11), 2316-2320.

Kim, S., Min, D., Lin, S., \& Sohn, K. (2020). Discrete-continuous transformation matching for dense semantic correspondence. IEEE Transactions on Pattern Analysis and Machine Intelligence, 42(1), 59-73.

Klein, S., Staring, M., \& Pluim, J. P. (2007). Evaluation of optimization methods for nonrigid medical image registration using mutual information and b-splines. IEEE Transactions on Image Processing, 16(12), 2879-2890.

Kluger, F., Brachmann, E., Ackermann, H., Rother, C., Yang, M. Y., \& Rosenhahn, B. (2020). Consac: Robust multi-model fitting by conditional sample consensus. In Proceedings of the IEEE/CVF conference on computer vision and pattern recognition, pp. 4634 4643.

Komorowski, J., Czarnota, K., Trzcinski, T., Dabala, L., \& Lynen, S. (2018). Interest point detectors stability evaluation on apolloscape dataset. In Proceedings of the European conference on computer vision, pp. 727-739.

Kovnatsky, A., Bronstein, M. M., Bresson, X., \& Vandergheynst, P. (2015). Functional correspondence by matrix completion. In Proceedings of the IEEE conference on computer vision and pattern recognition, pp. 905-914.

Krebs, J., Mansi, T., Delingette, H., Zhang, L., Ghesu, F. C., Miao, S., Maier, A. K., Ayache, N., Liao, R., \& Kamen, A. (2017). 
Robust non-rigid registration through agent-based action learning. In Proceedings of the international conference on medical image computing and computer-assisted intervention, pp. 344-352.

Kulis, B., \& Darrell, T. (2009). Learning to hash with binary reconstructive embeddings. In Advances in neural information processing systems, pp. 1042-1050.

Kulis, B., \& Grauman, K. (2009). Kernelized locality-sensitive hashing for scalable image search. In Proceedings of the IEEE international conference on computer vision, pp. 2130-2137.

Kumar, B., Carneiro, G., Reid, I., et al. (2016). Learning local image descriptors with deep siamese and triplet convolutional networks by minimising global loss functions. In Proceedings of the IEEE conference on computer vision and pattern recognition, pp. 5385 5394.

Laskar, Z., \& Kannala, J. (2018). Semi-supervised semantic matching. In Proceedings of the European conference on computer vision workshop, pp. 1-11.

Lawin, F. J., Danelljan, M., Khan, F., Forssén, P. E., \& Felsberg, M. (2018). Density adaptive point set registration. In Proceedings of the IEEE international conference on computer vision, pp. 38293837.

Lawler, E. L. (1963). The quadratic assignment problem. Management Science, 9(4), 586-599.

Lazaridis, G., \& Petrou, M. (2006). Image registration using the Walsh transform. IEEE Transactions on Image Processing, 15(8), 23432357.

Le Moigne, J., Campbell, W. J., \& Cromp, R. F. (2002). An automated parallel image registration technique based on the correlation of wavelet features. IEEE Transactions on Geoscience and Remote Sensing, 40(8), 1849-1864.

Lebeda, K., Matas, J., \& Chum, O. (2012). Fixing the locally optimized ransac-full experimental evaluation. In Proceedings of the British machine vision conference, $\mathrm{pp} .1-11$.

Lee, J., Cho, M., \& Lee, K. M. (2010). A graph matching algorithm using data-driven markov chain monte carlo sampling. In Proceedings of the international conference on pattern recognition, pp. 2816 2819.

Lee, J., Cho, M., \& Lee, K. M. (2011). Hyper-graph matching via reweighted random walks. In Proceedings of the IEEE conference on computer vision and pattern recognition, pp. 1633-1640.

Lee, S., Lim, J., \& Suh, I. H. (2020). Progressive feature matching: Incremental graph construction and optimization. In IEEE transactions on image processing.

Lê-Huu, D. K., \& Paragios, N. (2017). Alternating direction graph matching. In Proceedings of the IEEE conference on computer vision and pattern recognition, pp. 4914-4922.

Lenc, K., \& Vedaldi, A. (2014). Large scale evaluation of local image feature detectors on homography datasets. In Proceedings of the British machine vision conference.

Lenc, K., \& Vedaldi, A. (2016). Learning covariant feature detectors. In Proceedings of the European conference on computer vision, pp. $100-117$.

Leordeanu, M., \& Hebert, M. (2005). A spectral technique for correspondence problems using pairwise constraints. In Proceedings of the IEEE international conference on computer vision, pp. 1482 1489.

Leordeanu, M., Hebert, M., \& Sukthankar, R. (2009). An integer projected fixed point method for graph matching and map inference. In Advances in neural information processing systems, pp. 11141122.

Leordeanu, M., Sukthankar, R., \& Hebert, M. (2012). Unsupervised learning for graph matching. International Journal of Computer Vision, 96(1), 28-45.

Leutenegger, S., Chli, M., \& Siegwart, R. (2011). Brisk: Binary robust invariant scalable keypoints. In Proceedings of the IEEE international conference on computer vision, pp. 2548-2555.
Levi, G. (1973). A note on the derivation of maximal common subgraphs of two directed or undirected graphs. Calcolo, 9(4), 341.

Li, H., \& Hartley, R. (2007). The 3d-3d registration problem revisited. In Proceedings of the IEEE international conference on computer vision, pp. 1-8.

Li, X., Han, K., Li, S., \& Prisacariu, V. A. (2020). Dual-resolution correspondence networks. arXiv preprint arXiv:2006.08844.

Li, H., Shen, T., \& Huang, X. (2009). Global optimization for alignment of generalized shapes. In Proceedings of the IEEE conference on computer vision and pattern recognition, pp. 856-863.

Li, H., Sumner, R. W., \& Pauly, M. (2008). Global correspondence optimization for non-rigid registration of depth scans. In Computer graphics forum, Vol. 27, Wiley Online Library, pp. 1421-1430.

Lian, W., Zhang, L., \& Yang, M. H. (2017). An efficient globally optimal algorithm for asymmetric point matching. IEEE Transactions on Pattern Analysis and Machine Intelligence, 39(7), 1281-1293.

Liao, R., Miao, S., de Tournemire, P., Grbic, S., Kamen, A., Mansi, T., \& Comaniciu, D. (2017). An artificial agent for robust image registration. In Proceedings of the thirty-first AAAI conference on artificial intelligence, pp. 4168-4175.

Liao, Q., Sun, D., \& Andreasson, H. (2020). Point set registration for $3 \mathrm{~d}$ range scans using fuzzy cluster-based metric and efficient global optimization. In IEEE transactions on pattern analysis and machine intelligence.

$\mathrm{Li}, \mathrm{X} .$, \& Hu, Z. (2010). Rejecting mismatches by correspondence function. International Journal of Computer Vision, 89(1), 1-17.

Li, Z., Mahapatra, D., Tielbeek, J. A., Stoker, J., van Vliet, L. J., \& Vos, F. M. (2015). Image registration based on autocorrelation of local structure. IEEE Transactions on Image Processing, 35(1), 63-75.

Lin, W. Y. D., Cheng, M. M., Lu, J., Yang, H., Do, M. N., \& Torr, P. (2014). Bilateral functions for global motion modeling. In Proceedings of the European conference on computer vision, pp. 341-356.

Lin, W. Y., Liu, S., Jiang, N., Do, M. N., Tan, P., \& Lu, J. (2016b). Repmatch: Robust feature matching and pose for reconstructing modern cities. In Proceedings of the European conference on computer vision, pp. 562-579.

Lin, W. Y., Liu, S., Matsushita, Y., Ng, T. T., \& Cheong, L. F. (2011). Smoothly varying affine stitching. In Proceedings of the IEEE conference on computer vision and pattern recognition, pp. 345-352.

Lin, K., Lu, J., Chen, C. S., \& Zhou, J. (2016a). Learning compact binary descriptors with unsupervised deep neural networks. In Proceedings of the IEEE conference on computer vision and pattern recognition, pp. 1183-1192.

Lindeberg, T. (1998). Feature detection with automatic scale selection. International Journal of Computer Vision, 30(2), 79-116.

Lin, W. Y., Wang, F., Cheng, M. M., Yeung, S. K., Torr, P. H., Do, M. N., et al. (2017). Code: Coherence based decision boundaries for feature correspondence. IEEE Transactions on Pattern Analysis and Machine Intelligence, 40(1), 34-47.

Lipman, Y., \& Funkhouser, T. (2009). Möbius voting for surface correspondence. ACM Transactions on Graphics, 28(3), 72.

Lipman, Y., Yagev, S., Poranne, R., Jacobs, D. W., \& Basri, R. (2014). Feature matching with bounded distortion. ACM Transactions on Graphics, 33(3), 26.

Litany, O., Remez, T., Rodolà, E., Bronstein, A., \& Bronstein, M. (2017). Deep functional maps: Structured prediction for dense shape correspondence. In Proceedings of the IEEE international conference on computer vision, pp. 5659-5667.

Litjens, G., Kooi, T., Bejnordi, B. E., Setio, A. A. A., Ciompi, F., Ghafoorian, M., et al. (2017). A survey on deep learning in medical image analysis. Medical Image Analysis, 42, 60-88.

Litman, R., \& Bronstein, A. M. (2014). Learning spectral descriptors for deformable shape correspondence. IEEE Transactions on Pattern Analysis and Machine Intelligence, 36(1), 171-180. 
Liu, H., \& Yan, S. (2010). Common visual pattern discovery via spatially coherent correspondences. In Proceedings of the IEEE conference on computer vision and pattern recognition, pp. 1609-1616.

Liu, Y., \& Zhang, H. (2012). Indexing visual features: Real-time loop closure detection using a tree structure. In Proceedings of the IEEE international conference on robotics and automation, pp. 36133618.

Liu, Y., Feng, R., \& Zhang, H. (2015a). Keypoint matching by outlier pruning with consensus constraint. In Proceedings of the IEEE international conference on robotics and automation, pp. 54815486.

Liu, W., Wang, J., Ji, R., Jiang, Y. G., \& Chang, S. F. (2012a). Supervised hashing with kernels. In Proceedings of the IEEE conference on computer vision and pattern recognition, pp. 2074-2081.

Liu, Y., Wang, C., Song, Z., \& Wang, M. (2018b). Efficient global point cloud registration by matching rotation invariant features through translation search. In Proceedings of the European conference on computer vision, pp. 448-463.

Liu, R., Yang, C., Sun, W., Wang, X., \& Li, H. (2020). Stereogan: Bridging synthetic-to-real domain gap by joint optimization of domain translation and stereo matching. In Proceedings of the IEEE/CVF conference on computer vision and pattern recognition, pp. 12,757-12,766.

Liu, X., Ai, Y., Zhang, J., \& Wang, Z. (2018a). A novel affine and contrast invariant descriptor for infrared and visible image registration. Remote Sensing, 10(4), 658.

Liu, Y., Chen, X., Peng, H., \& Wang, Z. (2017). Multi-focus image fusion with a deep convolutional neural network. Information Fusion, 36, 191-207.

Liu, H., Guo, B., \& Feng, Z. (2005). Pseudo-log-polar Fourier transform for image registration. IEEE Signal Processing Letters, 13(1), 1720.

Liu, Y., Liu, S., \& Wang, Z. (2015b). Multi-focus image fusion with dense sift. Information Fusion, 23, 139-155.

Liu, M., Pradalier, C., \& Siegwart, R. (2013). Visual homing from scale with an uncalibrated omnidirectional camera. IEEE Transactions on Robotics, 29(6), 1353-1365.

Liu, Z. Y., \& Qiao, H. (2014). GNCCP-graduated nonconvexityand concavity procedure. IEEE Transactions on Pattern Analysis and Machine Intelligence, 36(6), 1258-1267.

Liu, Z. Y., Qiao, H., \& Xu, L. (2012b). An extended path following algorithm for graph-matching problem. IEEE Transactions on Pattern Analysis and Machine Intelligence, 34(7), 1451-1456.

Li, Y., Wang, S., Tian, Q., \& Ding, X. (2015). A survey of recent advances in visual feature detection. Neurocomputing, 149, 736751.

Loeckx, D., Slagmolen, P., Maes, F., Vandermeulen, D., \& Suetens, P. (2009). Nonrigid image registration using conditional mutual information. IEEE Transactions on Image, 29(1), 19-29.

Loiola, E. M., de Abreu, N. M. M., Boaventura-Netto, P. O., Hahn, P., \& Querido, T. (2007). A survey for the quadratic assignment problem. European Journal of Operational Research, 176(2), 657-690.

Lowe, D.G., et al. (1999). Object recognition from local scale-invariant features. In Proceedings of the IEEE international conference on computer vision, pp. 1150-1157.

Lowe, D. G. (2004). Distinctive image features from scale-invariant keypoints. International Journal of Computer Vision, 60(2), 91110 .

Lowry, S., \& Andreasson, H. (2018). Logos: Local geometric support for high-outlier spatial verification. In Proceedings of the IEEE international conference on robotics and automation, pp. 72627269.

Luo, W., Schwing, A. G., \& Urtasun, R. (2016). Efficient deep learning for stereo matching. In Proceedings of the IEEE conference on computer vision and pattern recognition, pp. 5695-5703.
Luo, Z., Shen, T., Zhou, L., Zhang, J., Yao, Y., Li, S., Fang, T., \& Quan, L. (2019). Contextdesc: Local descriptor augmentation with cross-modality context. In Proceedings of the IEEE conference on computer vision and pattern recognition, pp. 2527-2536.

Luo, Z., Zhou, L., Bai, X., Chen, H., Zhang, J., Yao, Y., Li, S., Fang, T., \& Quan, L. (2020). Aslfeat: Learning local features of accurate shape and localization. In Proceedings of the IEEE/CVF conference on computer vision and pattern recognition, pp. 6589-6598.

Ma, J., Zhao, J., Jiang, J., Zhou, H., Zhou, Y., Wang, Z., \& Guo, X. (2018b). Visual homing via guided locality preserving matching. In Proceedings of the IEEE international conference on robotics and automation, pp. 7254-7261.

Ma, J., Zhao, J., Tian, J., Tu, Z., \& Yuille, A. L. (2013b). Robust estimation of nonrigid transformation for point set registration. In Proceedings of the IEEE conference on computer vision and pattern recognition, pp. 2147-2154.

Ma, J., Chen, C., Li, C., \& Huang, J. (2016a). Infrared and visible image fusion via gradient transfer and total variation minimization. Information Fusion, 31, 100-109.

Maes, F., Collignon, A., Vandermeulen, D., Marchal, G., \& Suetens, P. (1997). Multimodality image registration by maximization of mutual information. IEEE Transactions on Image, 16(2), 187-198.

Mainali, P., Lafruit, G., Yang, Q., Geelen, B., Van Gool, L., \& Lauwereins, R. (2013). Sifer: Scale-invariant feature detector with error resilience. International Journal of Computer Vision, 104(2), 172197.

Mair, E., Hager, G. D., Burschka, D., Suppa, M., \& Hirzinger, G. (2010). Adaptive and generic corner detection based on the accelerated segment test. In Proceedings of the European conference on computer vision, pp. 183-196.

Maiseli, B., Gu, Y., \& Gao, H. (2017). Recent developments and trends in point set registration methods. Journal of Visual Communication and Image Representation, 46, 95-106.

Ma, J., Jiang, X., Jiang, J., Zhao, J., \& Guo, X. (2019a). LMR: Learning a two-class classifier for mismatch removal. IEEE Transactions on Image Processing, 28(8), 4045-4059.

Ma, J., Jiang, J., Liu, C., \& Li, Y. (2017a). Feature guided Gaussian mixture model with semi-supervised em and local geometric constraint for retinal image registration. Information Sciences, 417, $128-142$.

Ma, J., Jiang, J., Zhou, H., Zhao, J., \& Guo, X. (2018a). Guided locality preserving feature matching for remote sensing image registration. IEEE Transactions on Geoscience and Remote Sensing, 56(8), 4435-4447.

Ma, J., Liang, P., Yu, W., Chen, C., Guo, X., Wu, J., et al. (2020). Infrared and visible image fusion via detail preserving adversarial learning. Information Fusion, 54, 85-98.

Ma, J., Qiu, W., Zhao, J., Ma, Y., Yuille, A. L., \& Tu, Z. (2015). Robust $l_{2} e$ estimation of transformation for non-rigid registration. IEEE Transactions on Signal Processing, 63(5), 1115-1129.

Marimon, D., Bonnin, A., Adamek, T., \& Gimeno, R. (2010). Darts: Efficient scale-space extraction of daisy keypoints. In Proceedings of the IEEE conference on computer vision and pattern recognition, pp. 2416-2423.

Maron, H., \& Lipman, Y. (2018). (probably) concave graph matching. In Advances in Neural information processing systems, pp. 406-416.

Maron, H., Dym, N., Kezurer, I., Kovalsky, S., \& Lipman, Y. (2016). Point registration via efficient convex relaxation. ACM Transactions on Graphics, 35(4), 73.

Masci, J., Boscaini, D., Bronstein, M., \& Vandergheynst, P. (2015). Geodesic convolutional neural networks on Riemannian manifolds. In Proceedings of the IEEE international conference on computer vision workshops, pp. 37-45.

Masood, A., \& Sarfraz, M. (2007). Corner detection by sliding rectangles along planar curves. Computers \& Graphics, 31(3), 440-448. 
Matas, J., Chum, O., Urban, M., \& Pajdla, T. (2004). Robust widebaseline stereo from maximally stable extremal regions. Image and Vision Computing, 22(10), 761-767.

Ma, W., Wen, Z., Wu, Y., Jiao, L., Gong, M., Zheng, Y., et al. (2017b). Remote sensing image registration with modified sift and enhanced feature matching. IEEE Geoscience and Remote Sensing Letters, 14(1), 3-7.

Ma, J., Wu, J., Zhao, J., Jiang, J., Zhou, H., \& Sheng, Q. Z. (2019b). Nonrigid point set registration with robust transformation learning under manifold regularization. IEEE Transactions on Neural Networks and Learning Systems, 30(12), 3584-3597.

Mayer, N., Ilg, E., Hausser, P., Fischer, P., Cremers, D., Dosovitskiy, A., \& Brox, T. (2016). A large dataset to train convolutional networks for disparity, optical flow, and scene flow estimation. In Proceedings of the IEEE conference on computer vision and pattern recognition, pp. 4040-4048.

Ma, J., Yu, W., Liang, P., Li, C., \& Jiang, J. (2019c). Fusiongan: A generative adversarial network for infrared and visible image fusion. Information Fusion, 48, 11-26.

Ma, J., Zhao, J., Jiang, J., Zhou, H., \& Guo, X. (2019d). Locality preserving matching. International Journal of Computer Vision, 127(5), $512-531$.

Ma, J., Zhao, J., Ma, Y., \& Tian, J. (2015a). Non-rigid visible and infrared face registration via regularized gaussian fields criterion. Pattern Recognition, 48(3), 772-784.

Ma, J., Zhao, J., Tian, J., Bai, X., \& Tu, Z. (2013a). Regularized vector field learning with sparse approximation for mismatch removal. Pattern Recognition, 46(12), 3519-3532.

Ma, J., Zhao, J., Tian, J., Yuille, A. L., \& Tu, Z. (2014). Robust point matching via vector field consensus. IEEE Transactions on Image Processing, 23(4), 1706-1721.

Ma, J., Zhao, J., \& Yuille, A. L. (2016b). Non-rigid point set registration by preserving global and local structures. IEEE Transactions on Image Processing, 25(1), 53-64.

Ma, J., Zhou, H., Zhao, J., Gao, Y., Jiang, J., \& Tian, J. (2015b). Robust feature matching for remote sensing image registration via locally linear transforming. IEEE Transactions on Geoscience and Remote Sensing, 53(12), 6469-6481.

Mian, A., Bennamoun, M., \& Owens, R. (2010). On the repeatability and quality of keypoints for local feature-based $3 \mathrm{~d}$ object retrieval from cluttered scenes. International Journal of Computer Vision, 89(2-3), 348-361.

Miao, S., Piat, S., Fischer, P., Tuysuzoglu, A., Mewes, P., Mansi, T., \& Liao, R. (2018). Dilated fen for multi-agent $2 \mathrm{~d} / 3 \mathrm{~d}$ medical image registration. In Proceedings of the thirty-second AAAI conference on artificial intelligence, pp. 4694-4701.

Mikolajczyk, K., \& Schmid, C. (2001). Indexing based on scale invariant interest points. In Proceedings of the IEEE international conference on computer vision, pp. 525-531.

Mikolajczyk, K., \& Schmid, C. (2004). Scale \& affine invariant interest point detectors. International Journal of Computer Vision, 60(1), 63-86.

Mikolajczyk, K., \& Schmid, C. (2005). A performance evaluation of local descriptors. IEEE Transactions on Pattern Analysis and Machine Intelligence, 27(10), 1615-1630.

Mikolajczyk, K., Tuytelaars, T., Schmid, C., Zisserman, A., Matas, J., Schaffalitzky, F., et al. (2005). A comparison of affine region detectors. International Journal of Computer Vision, 65(1-2), 43-72.

Mishchuk, A., Mishkin, D., Radenovic, F., \& Matas, J. (2017). Working hard to know your neighbor's margins: Local descriptor learning loss. In Advances in neural information processing systems, pp. $4826-4837$.

Mishkin, D., Radenovic, F., \& Matas, J. (2017). Learning discriminative affine regions via discriminability. arXiv preprint arXiv:1711.06704.
Mishkin, D., Radenovic, F., \& Matas, J. (2018). Repeatability is not enough: Learning affine regions via discriminability. In Proceedings of the European conference on computer vision, pp. 284-300.

Mitra, R., Doiphode, N., Gautam, U., Narayan, S., Ahmed, S., Chandran, S., \& Jain, A. (2018). A large dataset for improving patch matching. arXiv preprint arXiv:1801.01466.

Mok, T. C., \& Chung, A. (2020). Fast symmetric diffeomorphic image registration with convolutional neural networks. In Proceedings of the IEEE/CVF conference on computer vision and pattern recognition, pp. 4644-4653.

Mokhtarian, F., \& Suomela, R. (1998). Robust image corner detection through curvature scale space. IEEE Transactions on Pattern Analysis and Machine Intelligence, 20(12), 1376-1381.

Möller, R., Krzykawski, M., \& Gerstmayr, L. (2010). Three 2d-warping schemes for visual robot navigation. Autonomous Robots, 29(3-4), 253-291.

Monti, F., Boscaini, D., Masci, J., Rodola, E., Svoboda, J., \& Bronstein, M. M. (2017). Geometric deep learning on graphs and manifolds using mixture model CNNs. In Proceedings of the IEEE conference on computer vision and pattern recognition, pp. 5115-5124.

Moo Yi, K., Trulls, E., Ono, Y., Lepetit, V., Salzmann, M., \& Fua, P. (2018). Learning to find good correspondences. In Proceedings of the IEEE conference on computer vision and pattern recognition, pp. 2666-2674.

Moo Yi, K., Verdie, Y., Fua, P., \& Lepetit, V. (2016). Learning to assign orientations to feature points. In Proceedings of the IEEE conference on computer vision and pattern recognition, pp. 107-116.

Moravec, H. P. (1977). Techniques towards automatic visual obstacle avoidance.

Morel, J. M., \& Yu, G. (2009). Asift: A new framework for fully affine invariant image comparison. SIAM Journal on Imaging Sciences, 2(2), 438-469.

Mukherjee, D., Wu, Q. J., \& Wang, G. (2015). A comparative experimental study of image feature detectors and descriptors. Machine Vision and Applications, 26(4), 443-466.

Mur-Artal, R., Montiel, J. M. M., \& Tardos, J. D. (2015). ORB-SLAM: A versatile and accurate monocular slam system. IEEE Transactions on Robotics, 31(5), 1147-1163.

Mustafa, A., Kim, H., \& Hilton, A. (2018). Msfd: Multi-scale segmentation-based feature detection for wide-baseline scene reconstruction. IEEE Transactions on Image Processing, 28(3), $1118-1132$.

Myronenko, A., \& Song, X. (2010). Point set registration: Coherent point drift. IEEE Transactions on Pattern Analysis and Machine Intelligence, 32(12), 2262-2275.

Nasuto, D., \& Craddock, J. B. R. (2002). Napsac: High noise, high dimensional robust estimation-it's in the bag. In Proceedings of the British machine vision conference, pp. 458-467.

Ni, K., Jin, H., \& Dellaert, F. (2009). Groupsac: Efficient consensus in the presence of groupings. In Proceedings of the IEEE international conference on computer vision, pp. 2193-2200.

Norouzi, M., \& Blei, D. M. (2011). Minimal loss hashing for compact binary codes. In Proceedings of the international conference on machine learning, pp. 353-360.

Nüchter, A., Lingemann, K., Hertzberg, J., \& Surmann, H. (2007). 6d SLAM-3d mapping outdoor environments. Journal of Field Robotics, 24(8-9), 699-722.

Ojala, T., Pietikäinen, M., \& Mäenpää, T. (2002). Multiresolution grayscale and rotation invariant texture classification with local binary patterns. IEEE Transactions on Pattern Analysis and Machine Intelligence, 24(7), 971-987.

Ono, Y., Trulls, E., Fua, P., \& Yi, K. M. (2018). LF-NET: Learning local features from images. In Advances in neural information processing systems, pp. 6234-6244. 
Ovsjanikov, M., Ben-Chen, M., Solomon, J., Butscher, A., \& Guibas, L. (2012). Functional maps: A flexible representation of maps between shapes. ACM Transactions on Graphics, 31(4), 30.

Pachauri, D., Kondor, R., \& Singh, V. (2013). Solving the multi-way matching problem by permutation synchronization. In Advances in neural information processing systems, pp. 1860-1868.

Pais, G. D., Ramalingam, S., Govindu, V. M., Nascimento, J. C., Chellappa, R., \& Miraldo, P. (2020). 3dregnet: A deep neural network for $3 \mathrm{~d}$ point registration. In Proceedings of the IEEE/CVF conference on computer vision and pattern recognition, pp. 7193-7203.

Pan, W. H., Wei, S. D., \& Lai, S. H. (2008). Efficient NCC-based image matching in Walsh-Hadamard domain. In Proceedings of the European conference on computer vision, pp. 468-480.

Pang, J., Sun, W., Ren, J. S., Yang, C., \& Yan, Q. (2017). Cascade residual learning: A two-stage convolutional neural network for stereo matching. In Proceedings of the IEEE international conference on computer vision, pp. 887-895.

Papazov, C., \& Burschka, D. (2011). Stochastic global optimization for robust point set registration. Computer Vision and Image Understanding, 115(12), 1598-1609.

Park, J., Zhou, Q. Y., \& Koltun, V. (2017). Colored point cloud registration revisited. In Proceedings of the IEEE international conference on computer vision, pp. 143-152.

Parra Bustos, A., Chin, T. J., \& Suter, D. (2014). Fast rotation search with stereographic projections for $3 \mathrm{~d}$ registration. In Proceedings of the IEEE conference on computer vision and pattern recognition, $\mathrm{pp}$. 3930-3937.

Paul, S., \& Pati, U. C. (2016). Remote sensing optical image registration using modified uniform robust sift. IEEE Geoscience and Remote Sensing Letters, 13(9), 1300-1304.

Perona, P., \& Malik, J. (1990). Scale-space and edge detection using anisotropic diffusion. IEEE Transactions on Pattern Analysis and Machine Intelligence, 12(7), 629-639.

Philbin, J., Chum, O., Isard, M., Sivic, J., \& Zisserman, A. (2007). Object retrieval with large vocabularies and fast spatial matching. In Proceedings of the IEEE conference on computer vision and pattern recognition, pp. 1-8.

Piasco, N., Sidibé, D., Demonceaux, C., \& Gouet-Brunet, V. (2018). A survey on visual-based localization: On the benefit of heterogeneous data. Pattern Recognition, 74, 90-109.

Pilet, J., Lepetit, V., \& Fua, P. (2008). Fast non-rigid surface detection, registration and realistic augmentation. International Journal of Computer Vision, 76(2), 109-122.

Pinheiro, A. M., \& Ghanbari, M. (2010). Piecewise approximation of contours through scale-space selection of dominant points. IEEE Transactions on Image Processing, 19(6), 1442-1450.

Plötz, T., \& Roth, S. (2018). Neural nearest neighbors networks. In Advances in Neural information processing systems, pp. 10871098.

Poggi, M., Pallotti, D., Tosi, F., \& Mattoccia, S. (2019). Guided stereo matching. In Proceedings of the IEEE conference on computer vision and pattern recognition, pp. 979-988.

Pohl, C., \& Van Genderen, J. L. (1998). Review article multisensor image fusion in remote sensing: concepts, methods and applications. International Journal of Remote Sensing, 19(5), 823-854.

Pokrass, J., Bronstein, A. M., Bronstein, M. M., Sprechmann, P., \& Sapiro, G. (2013). Sparse modeling of intrinsic correspondences. In Computer graphics forum, Vol. 32, Wiley Online Library, pp. 459-468.

Pomerleau, F., Colas, F., Siegwart, R., \& Magnenat, S. (2013). Comparing ICP variants on real-world data sets. Autonomous Robots, 34(3), 133-148.

Poursaeed, O., Yang, G., Prakash, A., Fang, Q., Jiang, H., Hariharan, B., $\&$ Belongie, S. (2018). Deep fundamental matrix estimation without correspondences. In Proceedings of the European conference on computer vision workshop, pp. 1-13.
Qi, C.R., Su, H., Mo, K., \& Guibas, L. J. (2017a). Pointnet: Deep learning on point sets for $3 \mathrm{~d}$ classification and segmentation. In Proceedings of the IEEE conference on computer vision and pattern recognition, pp. 652-660.

Qi, C. R., Yi, L., Su, H., \& Guibas, L. J. (2017b). Pointnet++: Deep hierarchical feature learning on point sets in a metric space. In Advances in neural information processing systems, pp. 50995108.

Quinlan, J. R. (1986). Induction of decision trees. Machine Learning, l(1), 81-106.

Raguram, R., Chum, O., Pollefeys, M., Matas, J., \& Frahm, J. M. (2012). USAC: A universal framework for random sample consensus. IEEE Transactions on Pattern Analysis and Machine Intelligence, 35(8), 2022-2038.

Ramer, U. (1972). An iterative procedure for the polygonal approximation of plane curves. Computer Graphics and Image Processing, 1(3), 244-256.

Ramisa, A., Goldhoorn, A., Aldavert, D., Toledo, R., \& de Mantaras, R. L. (2011). Combining invariant features and the ALV homing method for autonomous robot navigation based on panoramas. Journal of Intelligent \& Robotic Systems, 64(3-4), 625-649.

Ranftl, R., \& Koltun, V. (2018). Deep fundamental matrix estimation. In Proceedings of the European conference on computer vision, pp. 284-299.

Reddy, B. S., \& Chatterji, B. N. (1996). An FFT-based technique for translation, rotation, and scale-invariant image registration. IEEE Transactions on Image Processing, 5(8), 1266-1271.

Revaud, J., Weinzaepfel, P., De Souza, C., Pion, N., Csurka, G., Cabon, Y., \& Humenberger, M. (2019). R2d2: Repeatable and reliable detector and descriptor. arXiv preprint arXiv:1906.06195.

Revaud, J., Weinzaepfel, P., Harchaoui, Z., \& Schmid, C. (2016). Deepmatching: Hierarchical deformable dense matching. International Journal of Computer Vision, 120(3), 300-323.

Richardson, A., \& Olson, E. (2013). Learning convolutional filters for interest point detection. In Proceedings of the IEEE international conference on robotics and automation, pp. 631-637.

Robertson, C., \& Fisher, R. B. (2002). Parallel evolutionary registration of range data. Computer Vision and Image Understanding, 87(13), 39-50.

Rocco, I., Arandjelovic, R., \& Sivic, J. (2017). Convolutional neural network architecture for geometric matching. In Proceedings of the IEEE conference on computer vision and pattern recognition, pp. 6148-6157.

Rocco, I., Cimpoi, M., Arandjelović, R., Torii, A., Pajdla, T., \& Sivic, J. (2018). Neighbourhood consensus networks. In Advances in neural information processing systems, pp. 1651-1662.

Rodola, E., Bronstein, A. M., Albarelli, A., Bergamasco, F., \& Torsello, A. (2012). A game-theoretic approach to deformable shape matching. In Proceedings of the IEEE conference on computer vision and pattern recognition, pp. 182-189.

Rodolà, E., Cosmo, L., Bronstein, M. M., Torsello, A., \& Cremers, D. (2017). Partial functional correspondence. In Computer graphics forum, Vol. 36, Wiley Online Library, pp. 222-236.

Rodolà, E., Rota Bulo, S., Windheuser, T., Vestner, M., \& Cremers, D. (2014). Dense non-rigid shape correspondence using random forests. In Proceedings of the IEEE conference on computer vision and pattern recognition, pp. 4177-4184.

Rodola, E., Torsello, A., Harada, T., Kuniyoshi, Y., \& Cremers, D. (2013). Elastic net constraints for shape matching. In Proceedings of the IEEE international conference on computer vision, $\mathrm{pp}$. 1169-1176.

Rosenfeld, A., \& Weszka, J. S. (1975). An improved method of angle detection on digital curves. IEEE Transactions on Computers, 100(9), 940-941. 
Rosten, E., \& Drummond, T. (2006). Machine learning for high-speed corner detection. In Proceedings of the European conference on computer vision, pp. 430-443.

Rosten, E., Porter, R., \& Drummond, T. (2010). Faster and better: A machine learning approach to corner detection. IEEE Transactions on Pattern Analysis and Machine Intelligence, 32(1), 105-119.

Rublee, E., Rabaud, V., Konolige, K., \& Bradski, G. R. (2011). Orb: An efficient alternative to sift or surf. In Proceedings of the IEEE international conference on computer vision, pp. 2564-2571.

Rustamov, R. M. (2007). Laplace-Beltrami eigenfunctions for deformation invariant shape representation. In Proceedings of the Eurographics symposium on geometry processing, pp. 225-233.

Rusu, R. B., Blodow, N., \& Beetz, M. (2009). Fast point feature histograms (fpfh) for $3 \mathrm{~d}$ registration. In Proceedings of the IEEE international conference on robotics and automation, pp. 32123217.

Rusu, R. B., Blodow, N., Marton, Z. C., \& Beetz, M. (2008). Aligning point cloud views using persistent feature histograms. In Proceedings of the IEEE/RSJ international conference on intelligent robots and systems, pp. 3384-3391.

Sahillioglu, Y., \& Yemez, Y. (2011). Coarse-to-fine combinatorial matching for dense isometric shape correspondence. In Computer graphics forum, Vol. 30, Wiley Online Library, pp. 1461-1470.

Salakhutdinov, R., \& Hinton, G. (2009). Semantic hashing. International Journal of Approximate Reasoning, 50(7), 969-978.

Salti, S., Lanza, A., \& Di Stefano, L. (2013). Keypoints from symmetries by wave propagation. In Proceedings of the IEEE conference on computer vision and pattern recognition, pp. 2898-2905.

Salti, S., Tombari, F., Spezialetti, R., \& Di Stefano, L. (2015). Learning a descriptor-specific $3 \mathrm{~d}$ keypoint detector. In Proceedings of the IEEE international conference on computer vision, pp. 2318 2326.

Sandhu, R., Dambreville, S., \& Tannenbaum, A. (2010). Point set registration via particle filtering and stochastic dynamics. IEEE Transactions on Pattern Analysis and Machine Intelligence, 32(8), 1459-1473.

Sarlin, P.E., DeTone, D., Malisiewicz, T., \& Rabinovich, A. (2020). Superglue: Learning feature matching with graph neural networks. In Proceedings of the IEEE/CVF conference on computer vision and pattern recognition, pp. 4938-4947.

Savinov, N., Seki, A., Ladicky, L., Sattler, T., \& Pollefeys, M. (2017). Quad-networks: Unsupervised learning to rank for interest point detection. In Proceedings of the IEEE conference on computer vision and pattern recognition, pp. 1822-1830.

Scarselli, F., Gori, M., Tsoi, A. C., Hagenbuchner, M., \& Monfardini, G. (2009). The graph neural network model. In TNN.

Schellewald, C., \& Schnörr, C. (2005). Probabilistic subgraph matching based on convex relaxation. In Proceedings of the international workshop on energy minimization methods in computer vision and pattern recognition, pp. 171-186.

Schonberger, J. L., \& Frahm, J. M. (2016). Structure-from-motion revisited. In Proceedings of the IEEE conference on computer vision and pattern recognition, pp. 4104-4113.

Schonberger, J. L., Hardmeier, H., Sattler, T., \& Pollefeys, M. (2017). Comparative evaluation of hand-crafted and learned local features. In Proceedings of the IEEE conference on computer vision and pattern recognition, pp. 1482-1491.

Schroeter, D., \& Newman, P. (2008). On the robustness of visual homing under landmark uncertainty. In Proceedings of the intelligent autonomous systems, pp. 278-287.

Scott, G. L., \& Longuet-Higgins, H. C. (1991). An algorithm for associating the features of two images. Proceedings of the Royal Society of London. Series B: Biological Sciences, 244(1309), 21-26.

Shah, R., Srivastava, V., \& Narayanan, P. (2015). Geometry-aware feature matching for structure from motion applications. In Proceed- ings of the IEEE winter conference on applications of computer vision, pp. 278-285.

Shaked, A., \& Wolf, L. (2017). Improved stereo matching with constant highway networks and reflective confidence learning. In Proceedings of the IEEE conference on computer vision and pattern recognition, pp. 4641-4650.

Shakhnarovich, G. (2005). Learning task-specific similarity. Ph.D. thesis, Massachusetts Institute of Technology.

Shapiro, L. S., \& Brady, J. M. (1992). Feature-based correspondence: An eigenvector approach. Image and Vision Computing, 10(5), 283-288.

Shen, X., Wang, C., Li, X., Yu, Z., Li, J., Wen, C., Cheng, M., \& He, Z. (2019). RF-NET: An end-to-end image matching network based on receptive field. In Proceedings of the IEEE conference on computer vision and pattern recognition, pp. 8132-8140.

Shi, J., \& Tomasi, C. (1993). Good features to track. Technical report, Cornell University.

Silva, L., Bellon, O. R. P., \& Boyer, K. L. (2005). Precision range image registration using a robust surface interpenetration measure and enhanced genetic algorithms. IEEE Transactions on Pattern Analysis and Machine Intelligence, 27(5), 762-776.

Simonovsky, M., Gutiérrez-Becker, B., Mateus, D., Navab, N., \& Komodakis, N. (2016). A deep metric for multimodal registration. In Proceedings of the international conference on medical image computing and computer-assisted intervention, pp. 10-18.

Simonyan, K., Vedaldi, A., \& Zisserman, A. (2014). Learning local feature descriptors using convex optimization. IEEE Transactions on Pattern Analysis and Machine Intelligence, 36(8), 1573-1585.

Simo-Serra, E., Trulls, E., Ferraz, L., Kokkinos, I., Fua, P., \& MorenoNoguer, F. (2015). Discriminative learning of deep convolutional feature point descriptors. In Proceedings of the IEEE international conference on computer vision, pp. 118-126.

Sipiran, I., \& Bustos, B. (2011). Harris 3d: A robust extension of the Harris operator for interest point detection on $3 \mathrm{~d}$ meshes. The Visual Computer, 27(11), 963.

Sivic, J., \& Zisserman, A. (2003). Video google: A text retrieval approach to object matching in videos. In Proceedings of the IEEE international conference on computer vision, pp. 1-8.

Smith, S. M., \& Brady, J. M. (1997). Susan: A new approach to low level image processing. International Journal of Computer Vision, 23(1), 45-78.

Sofka, M., Yang, G., \& Stewart, C. V. (2007). Simultaneous covariance driven correspondence (CDC) and transformation estimation in the expectation maximization framework. In Proceedings of the IEEE conference on computer vision and pattern recognition, pp. 1-8.

Sokooti, H., de Vos, B., Berendsen, F., Lelieveldt, B. P., Isgum, I., \& Staring, M. (2017). Nonrigid image registration using multi-scale $3 \mathrm{~d}$ convolutional neural networks. In Proceedings of the international conference on medical image computing and computer-assisted intervention, pp. 232-239.

Sotiras, A., Davatzikos, C., \& Paragios, N. (2013). Deformable medical image registration: A survey. IEEE Transactions on Medical Imaging, 32(7), 1153.

Strecha, C., Lindner, A., Ali, K., \& Fua, P. (2009). Training for task specific keypoint detection. In Joint pattern recognition symposium, Springer, pp. 151-160.

Strecha, C., Von Hansen, W., Van Gool, L., Fua, P., \& Thoennessen, U. (2008). On benchmarking camera calibration and multi-view stereo for high resolution imagery. In Proceedings of the IEEE Conference on computer vision and pattern recognition, pp. 1-8.

Strecha, C., Bronstein, A., Bronstein, M., \& Fua, P. (2012). Ldahash: Improved matching with smaller descriptors. IEEE Transactions on Pattern Analysis and Machine Intelligence, 34(1), 66-78.

Sturm, J., Engelhard, N., Endres, F., Burgard, W., \& Cremers, D. (2012). A benchmark for the evaluation of RGB-D slam systems. In Pro- 
ceedings of the IEEE/RSJ international conference on intelligent robots and systems, pp. 573-580.

Suh, Y., Cho, M., \& Lee, K. M. (2012). Graph matching via sequential Monte Carlo. In Proceedings of the European conference on computer vision, pp. 624-637.

Sun, J., Ovsjanikov, M., \& Guibas, L. (2009). A concise and provably informative multi-scale signature based on heat diffusion. In Computer graphics forum, Vol. 28, Wiley Online Library, pp. 1383-1392.

Sweeney, C., Hollerer, T., \& Turk, M. (2015). Theia: A fast and scalable structure-from-motion library. In Proceedings of the ACM international conference on multimedia, pp. 693-696.

Swoboda, P., Kuske, J., \& Savchynskyy, B. (2017). A dual ascent framework for Lagrangean decomposition of combinatorial problems. In Proceedings of the IEEE conference on computer vision and pattern recognition, pp. 1596-1606.

Swoboda, P., Mokarian, A., Theobalt, C., Bernard, F., et al. (2019). A convex relaxation for multi-graph matching. In Proceedings of the IEEE conference on computer vision and pattern recognition, $\mathrm{pp}$. $11,156-11,165$.

Swoboda, P., Rother, C., Abu Alhaija, H., Kainmuller, D., \& Savchynskyy, B. (2017). A study of lagrangean decompositions and dual ascent solvers for graph matching. In Proceedings of the IEEE conference on computer vision and pattern recognition, pp. 16071616.

Takita, K., Aoki, T., Sasaki, Y., Higuchi, T., \& Kobayashi, K. (2003). High-accuracy subpixel image registration based on phase-only correlation. IEICE Transactions on Fundamentals of Electronics, Communications and Computer Sciences, 86(8), 1925-1934.

Tang, F., Lim, S. H., Chang, N. L., \& Tao, H. (2009). A novel feature descriptor invariant to complex brightness changes. In Proceedings of the IEEE conference on computer vision and pattern recognition, pp. 2631-2638.

Tevs, A., Berner, A., Wand, M., Ihrke, I., \& Seidel, H. P. (2011). Intrinsic shape matching by planned landmark sampling. In Computer graphics forum, Vol. 30, Wiley Online Library, pp. 543-552.

Thomee, B., Shamma, D. A., Friedland, G., Elizalde, B., Ni, K., Poland, D., et al. (2016). Yfcc100m: The new data in multimedia research. Communications of the ACM, 59(2), 64-73.

Tian, Y., Fan, B., \& Wu, F. (2017). L2-net: Deep learning of discriminative patch descriptor in Euclidean space. In Proceedings of the IEEE conference on computer vision and pattern recognition, pp. 661-669.

Tian, Y., Yan, J., Zhang, H., Zhang, Y., Yang, X., \& Zha, H. (2012). On the convergence of graph matching: Graduated assignment revisited. In Proceedings of the European conference on computer vision, pp. 821-835.

Tian, Y., Yu, X., Fan, B., Wu, F., Heijnen, H., \& Balntas, V. (2019). Sosnet: Second order similarity regularization for local descriptor learning. In Proceedings of the IEEE conference on computer vision and pattern recognition, pp. 11,016-11,025.

Toews, M., \& Wells, W. (2009). Sift-rank: Ordinal description for invariant feature correspondence. In Proceedings of the IEEE conference on computer vision and pattern recognition, pp. 172-177.

Tola, E., Lepetit, V., \& Fua, P. (2010). Daisy: An efficient dense descriptor applied to wide-baseline stereo. IEEE Transactions on Pattern Analysis and Machine Intelligence, 32(5), 815-830.

Tombari, F., Salti, S., \& Di Stefano, L. (2010a). Unique shape context for $3 \mathrm{~d}$ data description. In Proceedings of the ACM workshop on 3 D object retrieval, pp. 57-62.

Tombari, F., Salti, S., \& Di Stefano, L. (2010b). Unique signatures of histograms for local surface description. In Proceedings of the European conference on computer vision, pp. 356-369.

Tombari, F., Salti, S., \& Di Stefano, L. (2013). Performance evaluation of $3 \mathrm{~d}$ keypoint detectors. International Journal of Computer Vision, 102(1-3), 198-220.
Torr, P. H. (2003). Solving Markov random fields using semi definite programming. In Proceeding of AISTATS, pp. 1-8.

Torr, P., \& Zisserman, A. (1998). Robust computation and parametrization of multiple view relations. In Proceedings of the international conference on computer vision, pp. 727-732.

Torresani, L., Kolmogorov, V., \& Rother, C. (2012). A dual decomposition approach to feature correspondence. IEEE Transactions on Pattern Analysis and Machine Intelligence, 35(2), 259-271.

Torr, P. H., \& Zisserman, A. (2000). Mlesac: A new robust estimator with application to estimating image geometry. Computer Vision and Image Understanding, 78(1), 138-156.

Trajković, M., \& Hedley, M. (1998). Fast corner detection. Image and Vision Computing, 16(2), 75-87.

Tron, R., Zhou, X., Esteves, C., \& Daniilidis, K. (2017). Fast multiimage matching via density-based clustering. In Proceedings of the IEEE international conference on computer vision, pp. 40574066.

Truong, P., Danelljan, M., \& Timofte, R. (2020). Glu-net: Global-local universal network for dense flow and correspondences. In Proceedings of the IEEE/CVF conference on computer vision and pattern recognition, pp. 6258-6268.

Trzcinski, T., \& Lepetit, V. (2012). Efficient discriminative projections for compact binary descriptors. In Proceedings of the European conference on computer vision, pp. 228-242.

Trzcinski, T., Christoudias, M., Fua, P., \& Lepetit, V. (2013). Boosting binary keypoint descriptors. In Proceedings of the IEEE conference on computer vision and pattern recognition, pp. 2874-2881.

Trzcinski, T., Christoudias, M., Lepetit, V., \& Fua, P. (2012). Learning image descriptors with the boosting-trick. In Advances in neural information processing systems, pp. 269-277.

Trzcinski, T., Christoudias, M., \& Lepetit, V. (2014). Learning image descriptors with boosting. IEEE Transactions on Pattern Analysis and Machine Intelligence, 37(3), 597-610.

Tsin, Y., \& Kanade, T. (2004). A correlation-based approach to robust point set registration. In Proceedings of the European conference on computer vision, pp. 558-569.

Tuytelaars, T., \& Van Gool, L. (2004). Matching widely separated views based on affine invariant regions. International Journal of Computer Vision, 59(1), 61-85.

Tuytelaars, T., Mikolajczyk, K., et al. (2008). Local invariant feature detectors: A survey. Foundations and Trends ${ }^{\circledR}$ in Computer Graphics and Vision, 3(3), 177-280.

Ufer, N., \& Ommer, B. (2017). Deep semantic feature matching. In Proceedings of the IEEE conference on computer vision and pattern recognition, pp. 6914-6923.

Umeyama, S. (1988). An eigen decomposition approach to weighted graph matching problems. IEEE Transactions on Pattern Analysis and Machine Intelligence, 10(5), 695-703.

Unnikrishnan, R., \& Hebert, M. (2008). Multi-scale interest regions from unorganized point clouds. In Proceedings of the IEEE conference on computer vision and pattern recognition workshops, pp. $1-8$.

van Wyk, B. J., \& van Wyk, M. A. (2004). A POCS-based graph matching algorithm. IEEE Transactions on Pattern Analysis and Machine Intelligence, 26(11), 1526-1530.

Van Kaick, O., Zhang, H., Hamarneh, G., \& Cohen-Or, D. (2011). A survey on shape correspondence. In Computer graphics forum, Vol. 30, Wiley Online Library, pp. 1681-1707.

Verdie, Y., Yi, K., Fua, P., \& Lepetit, V. (2015). Tilde: A temporally invariant learned detector. In Proceedings of the IEEE conference on computer vision and pattern recognition, pp. 5279-5288.

Vongkulbhisal, J., De la Torre, F., \& Costeira, J. P. (2017). Discriminative optimization: Theory and applications to point cloud registration. In Proceedings of the IEEE conference on computer vision and pattern recognition, pp. 4104-4112. 
Vongkulbhisal, J., Irastorza Ugalde, B., De la Torre, F., \& Costeira, J. P. (2018). Inverse composition discriminative optimization for point cloud registration. In Proceedings of the IEEE conference on computer vision and pattern recognition, pp. 2993-3001.

Wang, J., \& Zhang, M. (2020). Deepflash: An efficient network for learning-based medical image registration. In Proceedings of the IEEE/CVF conference on computer vision and pattern recognition, pp. 4444-4452.

Wang, C., Bronstein, M. M., Bronstein, A. M., \& Paragios, N. (2011). Discrete minimum distortion correspondence problems for non-rigid shape matching. In Proceedings of the international conference on scale space and variational methods in computer vision, pp. 580-591.

Wang, Z., Fan, B., \& Wu, F. (2011). Local intensity order pattern for feature description. In Proceedings of the international conference on computer vision, pp. 603-610.

Wang, H., Guo, J., Yan, D. M., Quan, W., \& Zhang, X. (2018b). Learning $3 \mathrm{~d}$ keypoint descriptors for non-rigid shape matching. In Proceedings of the European conference on computer vision, pp. 3-19.

Wang, J., Kumar, S., \& Chang, S. F. Semi-supervised hashing for scalable image retrieval. In Proceedings of the IEEE conference on computer vision and pattern recognition.

Wang, T., Liu, H., Li, Y., Jin, Y., Hou, X., \& Ling, H. (2020). Learning combinatorial solver for graph matching. In Proceedings of the IEEE/CVF conference on computer vision and pattern recognition, pp. 7568-7577.

Wang, J., Song, Y., Leung, T., Rosenberg, C., Wang, J., Philbin, J., Chen, B., \& Wu, Y. (2014). Learning fine-grained image similarity with deep ranking. In Proceedings of the IEEE conference on computer vision and pattern recognition, pp. 1386-1393.

Wang, G., Wang, Z., Chen, Y., Zhou, Q., \& Zhao, W. (2016). Contextaware Gaussian fields for non-rigid point set registration. In Proceedings of the IEEE conference on computer vision and pattern recognition, pp. 5811-5819.

Wang, F., Xue, N., Yu, J. G., \& Xia, G. S. (2020). Zero-assignment constraint for graph matching with outliers. In Proceedings of the IEEE/CVF conference on computer vision and pattern recognition, pp. 3033-3042.

Wang, F., Xue, N., Zhang, Y., Bai, X., \& Xia, G. S. (2018a). Adaptively transforming graph matching. In Proceedings of the European conference on computer vision, pp. 625-640.

Wang, R., Yan, J., \& Yang, X. (2019). Learning combinatorial embedding networks for deep graph matching. In ICCV.

Wang, Q., Zhou, X., \& Daniilidis, K. (2018). Multi-image semantic matching by mining consistent features. In Proceedings of the IEEE conference on computer vision and pattern recognition, $\mathrm{pp}$. 685-694.

Wang, J., Zhou, F., Wen, S., Liu, X., \& Lin, Y. (2017). Deep metric learning with angular loss. In Proceedings of the IEEE international conference on computer vision, pp. 2593-2601.

Wang, Z., Fan, B., Wang, G., \& Wu, F. (2015). Exploring local and overall ordinal information for robust feature description. IEEE Transactions on Pattern Analysis and Machine Intelligence, 38(11), 2198-2211.

Wang, G., Wang, Z., Chen, Y., \& Zhao, W. (2015). Robust point matching method for multimodal retinal image registration. Biomedical Signal Processing and Control, 19, 68-76.

Wei, L., Huang, Q., Ceylan, D., Vouga, E., \& Li, H. (2016). Dense human body correspondences using convolutional networks. In Proceedings of the IEEE conference on computer vision and pattern recognition, pp. 1544-1553.

Wei, X., Zhang, Y., Gong, Y., \& Zheng, N. (2018). Kernelized subspace pooling for deep local descriptors. In Proceedings of the IEEE conference on computer vision and pattern recognition, pp. 18671875 .
Weinberger, K. Q., \& Saul, L. K. (2009). Distance metric learning for large margin nearest neighbor classification. Journal of Machine Learning Research, 10(Feb), 207-244.

Weiss, Y., Torralba, A., \& Fergus, R. (2009) Spectral hashing. In Advances in neural information processing systems, pp. 17531760.

Windheuser, T., Vestner, M., Rodolà, E., Triebel, R., \& Cremers, D. (2014). Optimal intrinsic descriptors for non-rigid shape analysis. In Proceedings of the British machine vision conference.

Wohlhart, P., \& Lepetit, V. (2015). Learning descriptors for object recognition and $3 \mathrm{~d}$ pose estimation. In Proceedings of the IEEE conference on computer vision and pattern recognition, pp. 31093118.

Wu, Y., Lim, J., \& Yang, M. H. (2015b). Object tracking benchmark. IEEE Transactions on Pattern Analysis and Machine Intelligence, 37(9), 1834-1848.

Wu, J., Zhang, H., \& Guan, Y. (2014). Visual loop closure detection by matching binary visual features using locality sensitive hashing. In Proceeding of the world congress on intelligent control and automation, pp. 940-945.

Wu, C. Visualsfm: A visual structure from motion system. Retrieved November 16, 2018 from http://ccwu.me/vsfm/doc.html.

Wu, G., Kim, M., Wang, Q., Munsell, B. C., \& Shen, D. (2015a). Scalable high-performance image registration framework by unsupervised deep feature representations learning. IEEE Transactions on Biomedical Engineering, 63(7), 1505-1516.

Xiao, J., Owens, A., \& Torralba, A. (2013). Sun3d: A database of big spaces reconstructed using SFM and object labels. In Proceedings of the IEEE international conference on computer vision, pp. $1625-1632$.

Xie, J., Wang, M., \& Fang, Y. (2016). Learned binary spectral shape descriptor for $3 \mathrm{~d}$ shape correspondence. In Proceedings of the IEEE conference on computer vision and pattern recognition, pp. 3309-3317.

Yan, J., Li, Y., Liu, W., Zha, H., Yang, X., \& Chu, S. M. (2014). Graduated consistency-regularized optimization for multi-graph matching. In Proceedings of the European conference on computer vision, pp. 407-422.

Yan, J., Ren, Z., Zha, H., \& Chu, S. (2016a). A constrained clustering based approach for matching a collection of feature sets. In Proceedings of the international conference on pattern recognition, pp. 3832-3837.

Yan, J., Tian, Y., Zha, H., Yang, X., Zhang, Y., \& Chu, S. M. (2013). Joint optimization for consistent multiple graph matching. In Proceedings of the IEEE international conference on computer vision, pp. 1649-1656.

Yan, J., Xu, H., Zha, H., Yang, X., Liu, H., \& Chu, S. (2015c). A matrix decomposition perspective to multiple graph matching. In Proceedings of the IEEE international conference on computer vision, pp. 199-207.

Yan, J., Yin, X. C., Lin, W., Deng, C., Zha, H., \& Yang, X. (2016b). A short survey of recent advances in graph matching. In Proceedings of the ACM on international conference on multimedia retrieval, pp. 167-174.

Yan, J., Zhang, C., Zha, H., Liu, W., Yang, X., \& Chu, S. M. (2015d). Discrete hyper-graph matching. In Proceedings of the IEEE conference on computer vision and pattern recognition, $\mathrm{pp}$. $1520-1528$.

Yan, J., Cho, M., Zha, H., Yang, X., \& Chu, S. M. (2015a). Multi-graph matching via affinity optimization with graduated consistency regularization. IEEE Transactions on Pattern Analysis and Machine Intelligence, 38(6), 1228-1242.

Yang, M., Wu, F., \& Li, W. (2020). Waveletstereo: Learning wavelet coefficients of disparity map in stereo matching. In Proceedings of the IEEE/CVF conference on computer vision and pattern recognition, pp. 12,885-12,894. 
Yang, X., Kwitt, R., Styner, M., \& Niethammer, M. (2017b). Quicksilver: Fast predictive image registration-a deep learning approach. NeuroImage, 158, 378-396.

Yang, J., Li, H., Campbell, D., \& Jia, Y. (2016). Go-ICP: A globally optimal solution to $3 \mathrm{~d}$ ICP point-set registration. IEEE Transactions on Pattern Analysis and Machine Intelligence, 38(11), 2241-2254.

Yang, K., Pan, A., Yang, Y., Zhang, S., Ong, S., \& Tang, H. (2017a). Remote sensing image registration using multiple image features. Remote Sensing, 9(6), 581.

Yan, J., Wang, J., Zha, H., Yang, X., \& Chu, S. (2015b). Consistencydriven alternating optimization for multigraph matching: A unified approach. IEEE Transactions on Image Processing, 24(3), 9941009.

Yao, Y., Deng, B., Xu, W., \& Zhang, J. (2020). Quasi-Newton solver for robust non-rigid registration. In Proceedings of the IEEE/CVF conference on computer vision and pattern recognition, pp. 76007609.

Ye, Y., Shan, J., Bruzzone, L., \& Shen, L. (2017). Robust registration of multimodal remote sensing images based on structural similarity. IEEE Transactions on Geoscience and Remote Sensing, 55(5), 2941-2958.

Yew, Z. J., \& Lee, G. H. (2020). RPM-NET: Robust point matching using learned features. In Proceedings of the IEEE/CVF conference on computer vision and pattern recognition, pp. 11,82411,833 .

Yi, K. M., Trulls, E., Lepetit, V., \& Fua, P. (2016). Lift: Learned invariant feature transform. In Proceedings of the European conference on computer vision, pp. 467-483.

Yin, Z., \& Shi, J. (2018). Geonet: Unsupervised learning of dense depth, optical flow and camera pose. In Proceedings of the IEEE conference on computer vision and pattern recognition, pp. 1983-1992.

Yu, T., Wang, R., Yan, J., \& Li, B. (2020a). Learning deep graph matching with channel-independent embedding and Hungarian attention. In International conference on learning representations.

Yu, T., Yan, J., \& Li, B. (2020b). Determinant regularization for gradient-efficient graph matching. In Proceedings of the IEEE/CVF conference on computer vision and pattern recognition, pp. 7123-7132.

Yu, T., Yan, J., Wang, Y., Liu, W., et al. (2018). Generalizing graph matching beyond quadratic assignment model. In Advances in neural information processing systems, pp. 861-871.

Zagoruyko, S., \& Komodakis, N. (2015). Learning to compare image patches via convolutional neural networks. In Proceedings of the IEEE conference on computer vision and pattern recognition, $\mathrm{pp}$. 4353-4361.

Zaharescu, A., Boyer, E., Varanasi, K., \& Horaud, R. (2009). Surface feature detection and description with applications to mesh matching. In Proceedings of the IEEE conference on computer vision and pattern recognition, pp. 373-380.

Zanfir, A., \& Sminchisescu, C. (2018). Deep learning of graph matching. In Proceedings of the IEEE conference on computer vision and pattern recognition, pp. 2684-2693.

Zaslavskiy, M., Bach, F., \& Vert, J. P. (2009). A path following algorithm for the graph matching problem. IEEE Transactions on Pattern Analysis and Machine Intelligence, 31(12), 2227-2242.

Zass, R., \& Shashua, A. (2008). Probabilistic graph and hypergraph matching. In Proceedings of the IEEE conference on computer vision and pattern recognition, IEEE, pp. 1-8.

Zbontar, J., \& LeCun, Y. (2015). Computing the stereo matching cost with a convolutional neural network. In Proceedings of the IEEE conference on computer vision and pattern recognition, pp. 15921599.

Zbontar, J., \& LeCun, Y. (2016). Stereo matching by training a convolution neural network to compare image patches. The Journal of Machine Learning Research, 17(1), 2287-2318.
Zeng, Z., Chan, T. H., Jia, K., \& Xu, D. (2012). Finding correspondence from multiple images via sparse and low-rank decomposition. In Proceedings of the European conference on computer vision, pp. 325-339.

Zeng, A., Song, S., Nießner, M., Fisher, M., Xiao, J., \& Funkhouser, T. (2017). 3dmatch: Learning local geometric descriptors from RGB-D reconstructions. In Proceedings of the IEEE conference on computer vision and pattern recognition, pp. 1802-1811.

Zeng, Y., Wang, C., Wang, Y., Gu, X., Samaras, D., \& Paragios, N. (2010). Dense non-rigid surface registration using high-order graph matching. In Proceedings of the IEEE conference on computer vision and pattern recognition, pp. 382-389.

Zhang, H. (2011). Borf: Loop-closure detection with scale invariant visual features. In Proceedings of the IEEE international conference on robotics and automation, pp. 3125-3130.

Zhang, L., \& Rusinkiewicz, S. (2018). Learning to detect features in texture images. In Proceedings of the IEEE conference on computer vision and pattern recognition, pp. 6325-6333.

Zhang, F., Prisacariu, V., Yang, R., \& Torr, P. H. (2019a). Ga-net: Guided aggregation net for end-to-end stereo matching. In Proceedings of the IEEE conference on computer vision and pattern recognition, pp. 185-194.

Zhang, Z., Shi, Q., McAuley, J., Wei, W., Zhang, Y., \& Van Den Hengel, A. (2016). Pairwise matching through max-weight bipartite belief propagation. In Proceedings of the IEEE conference on computer vision and pattern recognition, pp. 1202-1210.

Zhang, J., Sun, D., Luo, Z., Yao, A., Zhou, L., Shen, T., Chen, Y., Quan, L., \& Liao, H. (2019b). Learning two-view correspondences and geometry using order-aware network. In Proceedings of the IEEE international conference on computer vision, pp. 5845-5854.

Zhang, S., Yang, Y., Yang, K., Luo, Y., \& Ong, S. H. (2017a). Point set registration with global-local correspondence and transformation estimation. In Proceedings of the IEEE international conference on computer vision, pp. 2669-2677.

Zhang, X., Yu, F. X., Karaman, S., \& Chang, S. F. (2017b). Learning discriminative and transformation covariant local feature detectors. In Proceedings of the IEEE conference on computer vision and pattern recognition, pp. 6818-6826.

Zhang, X., Yu, F. X., Kumar, S., \& Chang, S. F. (2017c). Learning spread-out local feature descriptors. In Proceedings of the IEEE international conference on computer vision, pp. 4595-4603.

Zhang, X., Qu, Y., Yang, D., Wang, H., \& Kymer, J. (2015). Laplacian scale-space behavior of planar curve corners. IEEE Transactions on Pattern Analysis and Machine Intelligence, 37(11), 2207-2217.

Zhang, X., Wang, H., Smith, A. W., Ling, X., Lovell, B. C., \& Yang, D. (2010). Corner detection based on gradient correlation matrices of planar curves. Pattern Recognition, 43(4), 1207-1223.

Zhao, J., \& Ma, J. (2017). Visual homing by robust interpolation for sparse motion flow. In Proceedings of the IEEE/RSJ international conference on intelligent robots and systems, pp. 1282-1288.

Zhao, C., Cao, Z., Li, C., Li, X., \& Yang, J. (2019). Nm-net: Mining reliable neighbors for robust feature correspondences. In Proceedings of the IEEE conference on computer vision and pattern recognition, pp. 215-224.

Zhao, Q., Karisch, S. E., Rendl, F., \& Wolkowicz, H. (1998). Semidefinite programming relaxations for the quadratic assignment problem. Journal of Combinatorial Optimization, 2(1), 71-109.

Zheng, L., Yang, Y., \& Tian, Q. (2018). Sift meets CNN: A decade survey of instance retrieval. IEEE Transactions on Pattern Analysis and Machine Intelligence, 40(5), 1224-1244.

Zhong, Y. (2009). Intrinsic shape signatures: A shape descriptor for $3 \mathrm{~d}$ object recognition. In Proceedings of the IEEE international conference on computer vision workshops, pp. 689-696.

Zhou, W., Li, H., \& Tian, Q. (2017). Recent advance in contentbased image retrieval: A literature survey. arXiv preprint arXiv:1706.06064. 
Zhou, W., Li, H., Lu, Y., \& Tian, Q. (2011). Large scale image search with geometric coding. In Proceedings of the ACM international conference on multimedia, pp. 1349-1352.

Zhou, W., Lu, Y., Li, H., Song, Y., \& Tian, Q. (2010). Spatial coding for large scale partial-duplicate web image search. In Proceedings of the ACM international conference on multimedia, pp. 511-520.

Zhou, X., Zhu, M., \& Daniilidis, K. (2015). Multi-image matching via fast alternating minimization. In Proceedings of the IEEE international conference on computer vision, pp. 4032-4040.

Zhou, L., Zhu, S., Luo, Z., Shen, T., Zhang, R., Zhen, M., Fang, T., \& Quan, L. (2018). Learning and matching multi-view descriptors for registration of point clouds. In Proceedings of the European conference on computer vision, pp. 505-522.

Zhou, F., \& De la Torre, F. (2015). Factorized graph matching. IEEE Transactions on Pattern Analysis and Machine Intelligence, 38(9), 1774-1789.

Zhu, J. Y., Park, T., Isola, P., \& Efros, A. A. (2017). Unpaired imageto-image translation using cycle-consistent adversarial networks. In Proceedings of the IEEE international conference on computer vision, pp. 2223-2232.

Zieba, M., Semberecki, P., El-Gaaly, T., \& Trzcinski, T. (2018). Bingan: Learning compact binary descriptors with a regularized GAN. In Advances in neural information processing systems, pp. 36083618.
Zitnick, C. L., \& Ramnath, K. (2011). Edge foci interest points. In Proceedings of the IEEE international conference on computer vision, pp. 359-366.

Zitova, B., \& Flusser, J. (2003). Image registration methods: A survey. Image and Vision Computing, 21(11), 977-1000.

Publisher's Note Springer Nature remains neutral with regard to jurisdictional claims in published maps and institutional affiliations. 